\title{
Synthesis of Sialyl Lewis ${ }^{\mathrm{x}}$ Glycomimetics Bearing a Bicyclic 3-O, 4-C-Fused Galactopyranoside Scaffold
}

Ryan D. Simard ${ }^{\dagger}$, Mathieu Joyal§, Laura Gillard ${ }^{\dagger}$, Gianna Di Censo ${ }^{\dagger}$, Wael Maharsy ${ }^{\S}$ Janie Beauregard $\$$, Pina Colarusso"l, Kamala D. Patel ${ }^{\|^{*}}$, Michel Prévost ${ }^{\dagger *}$, Mona Nemer ${ }^{\S}$, and Yvan Guindon,,$+ \AA^{*}$

†Bio-organic Chemistry Laboratory, Institut de recherches cliniques de Montréal (IRCM), Montréal, Québec, H2W 1R7, Canada

\$Department of Chemistry, Université de Montréal, Montréal, Québec, H3C 3J7, Canada

$\S$ Department of Biochemistry, Microbiology and Immunology, University of Ottawa, Ottawa, Ontario, K1N 6N5, Canada

"Live Cell Imaging Laboratory, Snyder Institute for Chronic Diseases, University of Calgary, Calgary, Alberta, T2N 4N1, Canada

Part I. Stereochemical Proofs S3

Part II. Computational Data .S5

Part III: ${ }^{1} \mathrm{H}$ and ${ }^{13} \mathrm{C}$ NMR Spectra S20

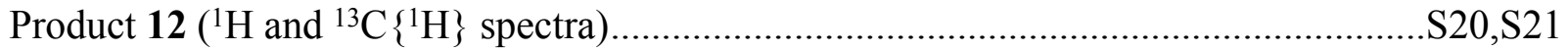

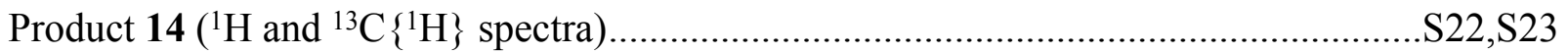

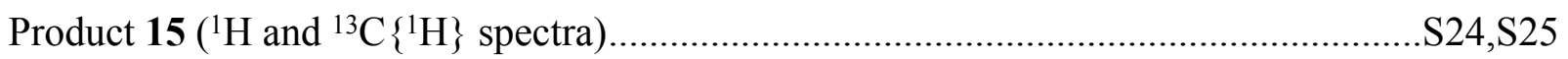

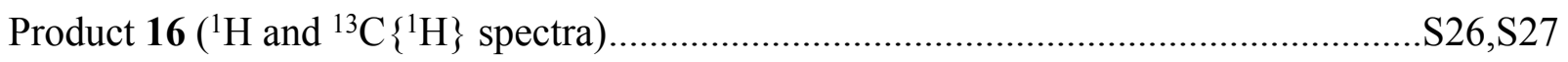

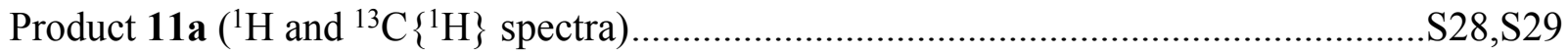

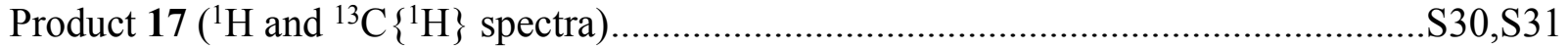

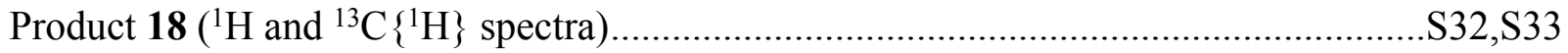

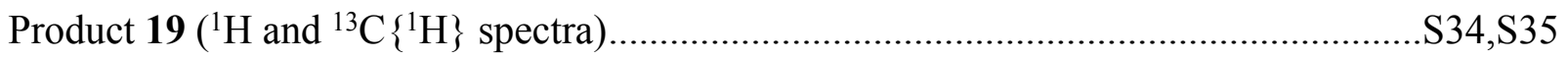

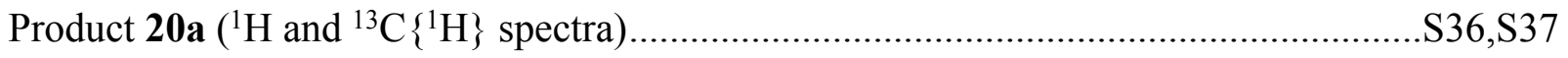

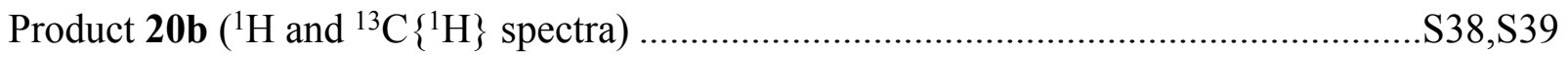

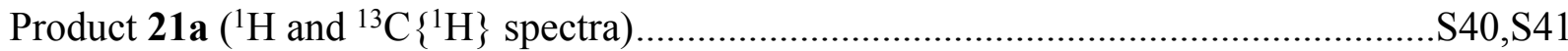

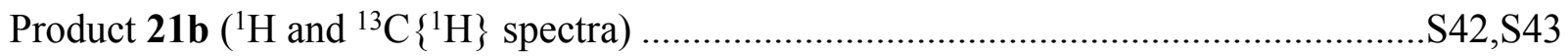

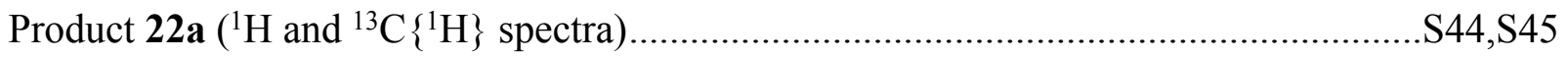

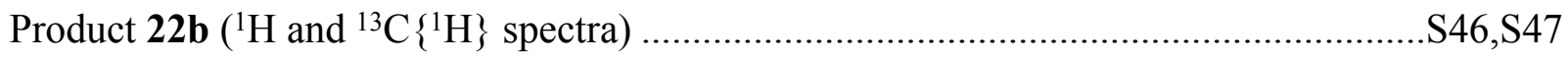

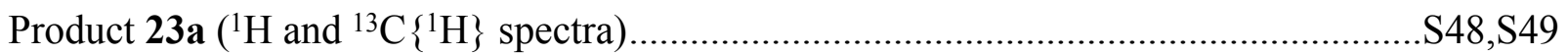

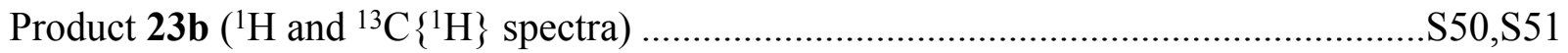

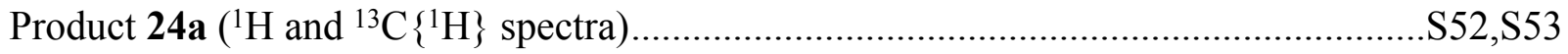

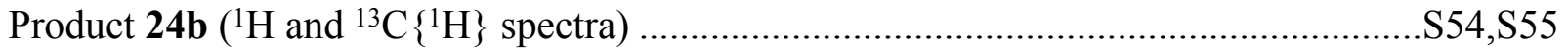




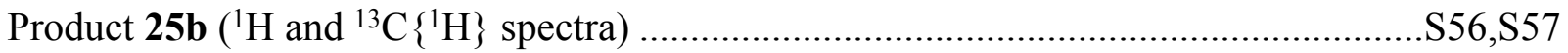

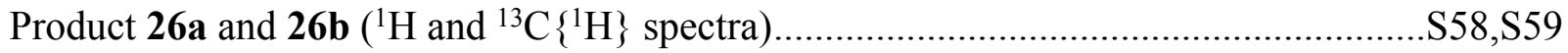

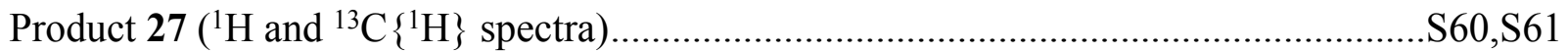

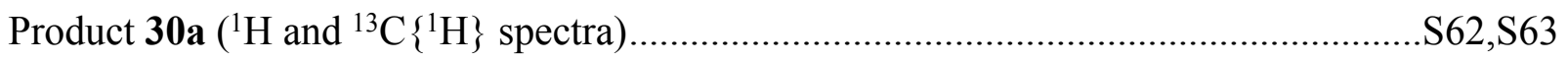

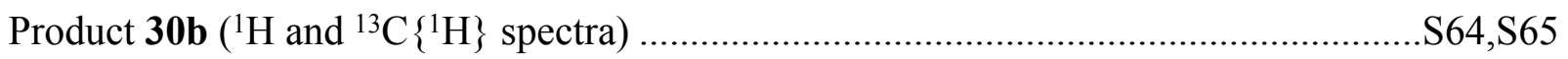

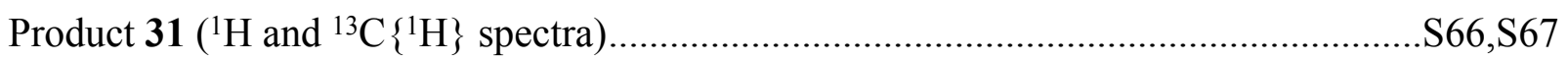

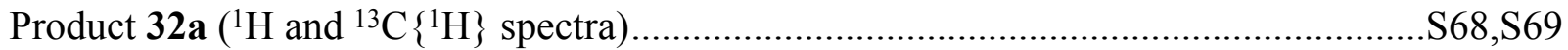

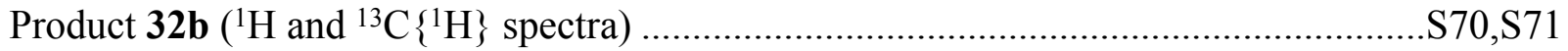

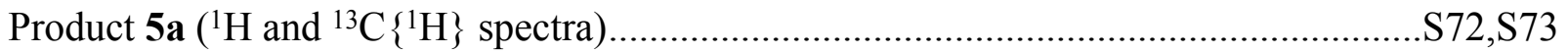

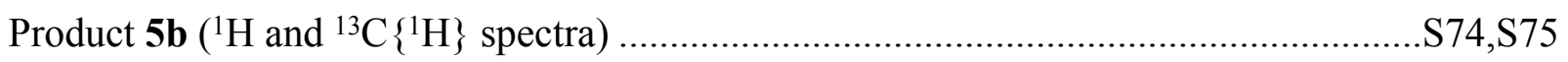

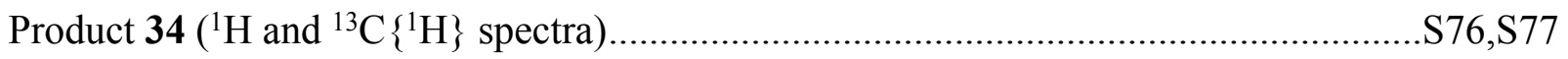

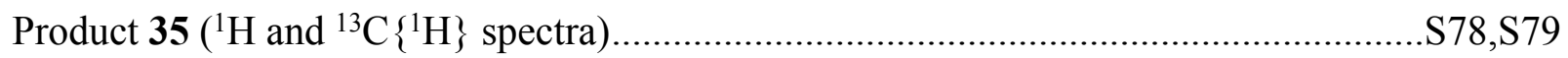

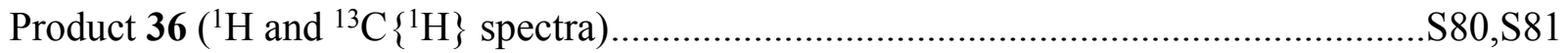

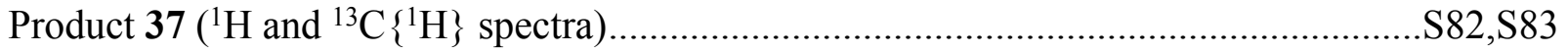

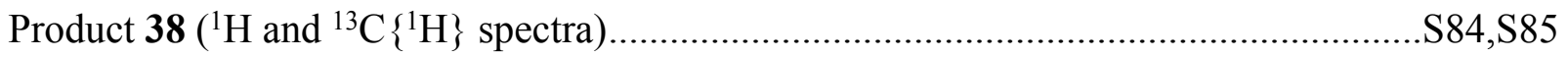

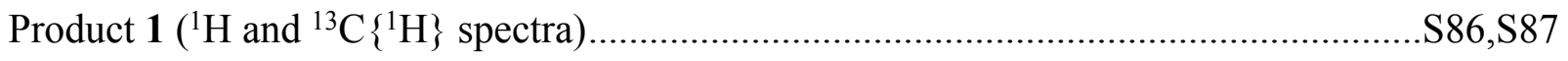

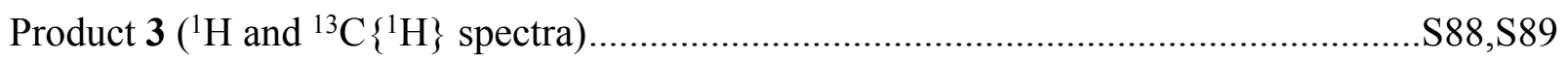

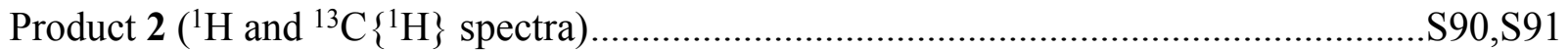

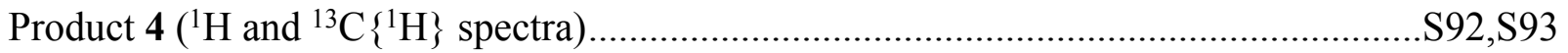

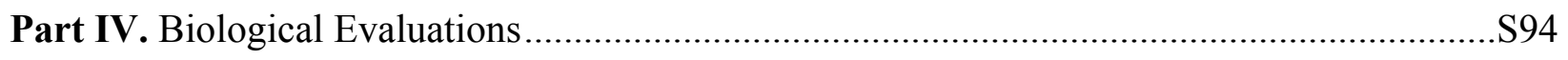

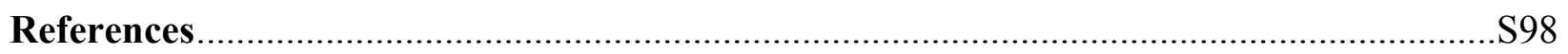




\section{Part I: Stereochemical Proofs}

In all cases, the selectivities were determined by ${ }^{1} \mathrm{H}$ NMR spectroscopic analysis of the unpurified reaction mixtures. The stereochemistry of the unsaturated $\alpha$-hydroxyesters, as well as the relative carboxylic acid configurations of the synthesized trans-bicyclo[4.4.0]galactopyranosides were determined by relevant nuclear Overhauser effect (NOE) enhancements (2D NOESY), ${ }^{1} \mathrm{H}$ NMR ${ }^{3} J_{\mathrm{H}, \mathrm{H}}$-coupling constant data and correlations of chemical shifts. The peaks in the ${ }^{1} \mathrm{H}$ NMR spectra were assigned using ${ }^{1} \mathrm{H} /{ }^{1} \mathrm{H}$ COSY experiments, chemical shifts, and coupling constants.

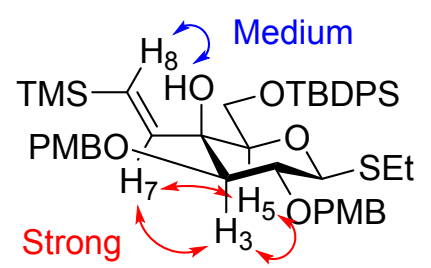

16

Relevant NOESY data for the $E: Z$ relative stereochemistry of galactoside $\mathbf{1 6}$.

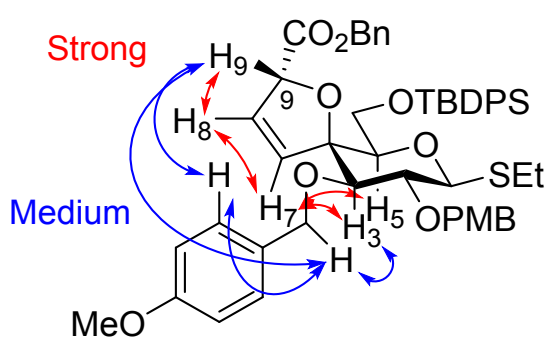

22a

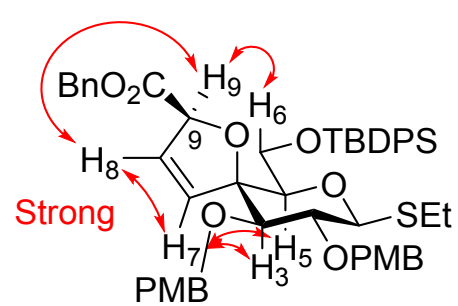

22b

Relevant NOESY data for the relative stereochemistry at C9 of spirocycles 22a and 22b 
${ }^{3} J_{\mathrm{H} 9, \mathrm{H} 8^{\prime}}=6.6 \mathrm{~Hz}$

${ }^{3} J_{\mathrm{H} 9, \mathrm{H} 8}=1.0 \mathrm{~Hz}$

${ }^{3} J_{\mathrm{H} 9, \mathrm{H} 8^{\prime}}=11.2 \mathrm{~Hz}$

${ }^{3} J_{\mathrm{H} 9, \mathrm{H} 8}=3.6 \mathrm{~Hz}$

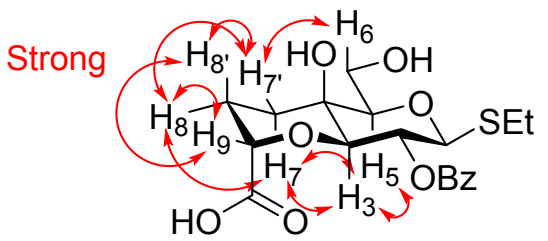

$5 b$

\section{From HSQC}

$\mathrm{H}_{3}(4.33 \mathrm{ppm}) \leftrightarrow \mathrm{C}_{3}$ (77.93 ppm)

$\mathrm{H}_{9}(4.21 \mathrm{ppm}) \leftrightarrow \mathrm{C}_{9}(76.03 \mathrm{ppm})$

From HMBC

$\mathrm{H}_{3}(4.33 \mathrm{ppm}) \longleftrightarrow \mathrm{C}_{9}(76.03 \mathrm{ppm})$ $\mathrm{H}_{9}(4.21 \mathrm{ppm}) \longleftrightarrow \mathrm{C}_{3}(77.93 \mathrm{ppm})$

\section{From HSQC}

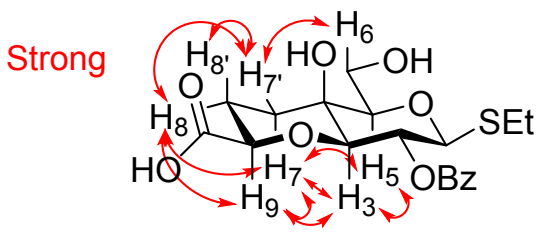

$5 a$

$\mathrm{H}_{3}(3.52 \mathrm{ppm}) \leftrightarrow \mathrm{C}_{3}(81.05 \mathrm{ppm})$ $\mathrm{H}_{9}(3.89 \mathrm{ppm}) \leftrightarrow \mathrm{C}_{9}(82.52 \mathrm{ppm})$

From HMBC

$\mathrm{H}_{3}(3.52 \mathrm{ppm}) \longleftrightarrow \mathrm{C}_{9}(82.52 \mathrm{ppm})$ $\mathrm{H}_{9}(3.89 \mathrm{ppm}) \longleftrightarrow \mathrm{C}_{3}(81.05 \mathrm{ppm})$

Relevant coupling constants, HSQC, HMBC and NOESY data for the relative configuration of the C9 carboxylic acid fragment of $\mathbf{5 a}$ and $\mathbf{5 b}$

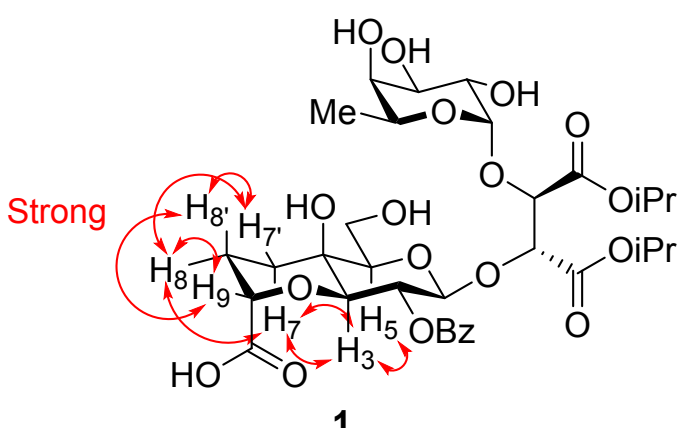

1

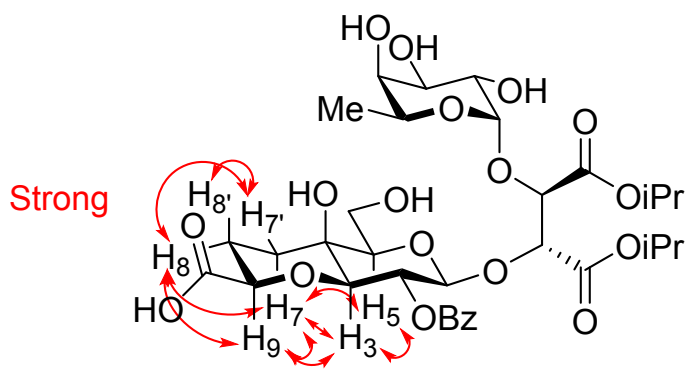

3

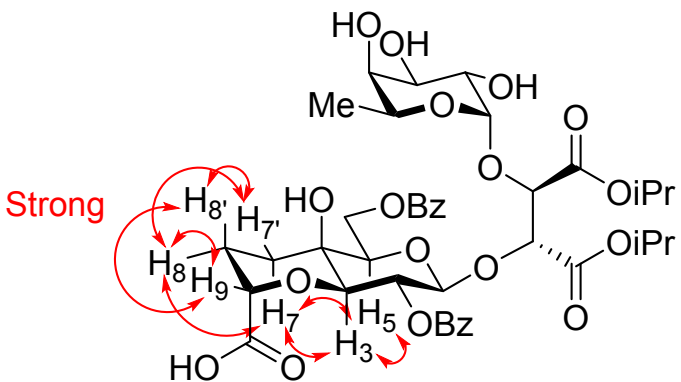

2

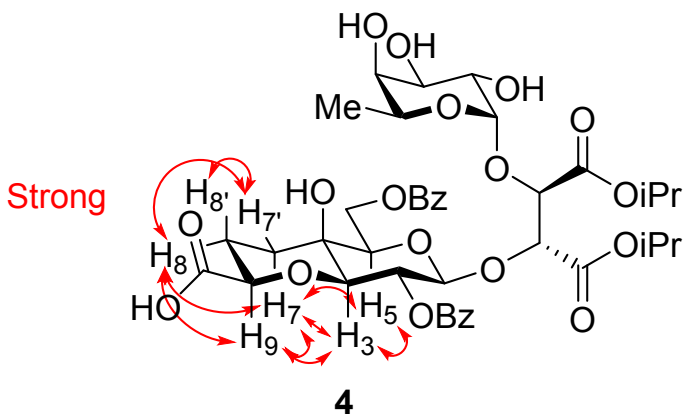

Relevant NOESY data confirming relative configuration of the C9 carboxylic acid fragment of compounds 1-4. 


\section{Part II: Computational Data}

\section{DFT Method and Details}

QM calculations were performed in Gaussian 09. ${ }^{[1]}$ All the possible conformations of the C1 SMe bond, the C2 and C3 hydroxyls, the C4-OTMS, and the $\beta, \gamma$-unsaturated methyl ester side chain where considered and optimized for the formation of the axial and equatorial diastereoisomers. The energies presented in the paper and the supporting information are from fully optimized structures at the M06-2X ${ }^{[2-3]}$ level of theory using the $6-31 G^{*}$ basis set for geometry optimization in conjunction with the $6-311+\mathrm{G}^{* *}$ basis set for single-point energies. Calculation in solvents (Toluene) were performed with transition state or geometry using the polarizable continuum model (PCM). ${ }^{[4]}$ Frequency calculations were performed at $353.15 \mathrm{~K}$ (1 atm) on every optimized structure to determine their nature, transition states or intermediates, and to determine free energy values $\left(1\right.$ Hartree $\left.=627.5095 \mathrm{kcal} \mathrm{mol}^{-1}\right)$. As mentioned in the manuscript, free energy corrections were applied using Truhlar's quasiharmonic approximation. [5-6] Gibbs free energies of activation $\left(\Delta \Delta \mathrm{G}^{\ddagger}\right)$ correspond to the energy difference between the lowest energy axial- and equatorial-predictive TS. 
Scheme S1: Gibbs Free Energy Profile for the Intramolecular Cyclization Reaction of Simplified Mesylate Precursors 28a,b to Form the trans-bicyclo[4.4.0]galactopyranosides 29a,b $(\underline{M 062 x / 6-31 G * / 6-311+G * *})$ at $\underline{353.15 \mathrm{~K}}$ in Toluene

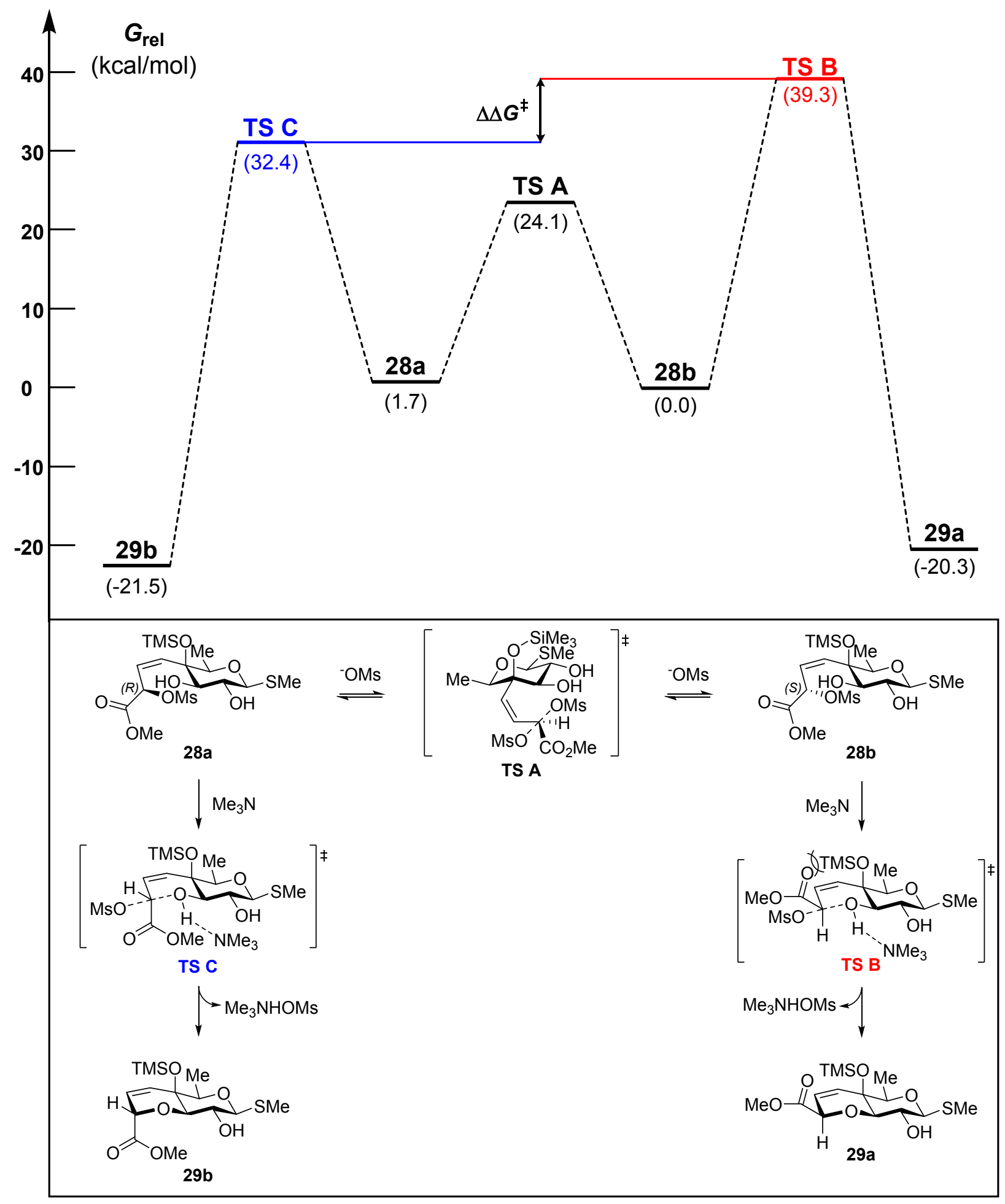


Free Gibbs Energies (Ha) Profile:

\begin{tabular}{|c|c|c|c|}
\hline \multirow{2}{*}{\multicolumn{2}{|c|}{ Species (Gibbs Free Energies Relative to $\mathbf{2 8 b}$ ) }} & \multicolumn{2}{|c|}{ Gibbs Free Energies } \\
\hline & & \multirow{2}{*}{\begin{tabular}{c|} 
(Ha) \\
-0.03228
\end{tabular}} & \multirow{2}{*}{$\begin{array}{c}(\mathrm{kcal} / \mathrm{mol}) \\
-20.3\end{array}$} \\
\hline $29 a$ & $\begin{array}{l}\text { 29a }(-1725.851615)+\text { MsOHNMe }_{3}(-838.614773)-[\mathbf{2 8 b}(-2390.093585)+ \\
\left.\text { NMe }_{3}(-174.340524)\right]\end{array}$ & & \\
\hline TS B & TS B (-2564.371506) - [28b (-2390.093585) + $\left.\mathbf{N M e}_{3}(-174.340524)\right]$ & 0.06260 & 39.3 \\
\hline $28 b$ & $\begin{array}{l}\mathbf{2 8 b}(-2390.093585) \text { and } \mathbf{N M e}_{3}(-174.340524) \text { (energies are represented relative } \\
\text { to this energy point) }\end{array}$ & $\mathbf{0}$ & $\mathbf{0}$ \\
\hline TS A & $\begin{array}{l}\text { TS A }(-3053.858402)+\mathbf{N M e}_{3}(-174.340524)-\mathbf{M s O}^{-}(-663.803227)-[\mathbf{2 8 b}(- \\
\left.2390.093585)+\mathbf{N M e}_{3}(-174.340524)\right]\end{array}$ & 0.03841 & 24.1 \\
\hline $28 a$ & $\begin{array}{l}\mathbf{2 8 a}(-2390.090930)+\mathbf{N M e}_{3}(-174.340524)-\left[\mathbf{2 8 b}(-2390.093585)+\mathbf{N M e}_{3}(-\right. \\
174.340524)]\end{array}$ & 0.002655 & 1.67 \\
\hline TS C & TS C (-2564.382511) - [28b (-2390.093585) + $\left.\mathbf{N M e}_{3}(-174.340524)\right]$ & 0.05160 & 32.4 \\
\hline 29b & $\begin{array}{l}\text { 29b }(-1725.853551)+\text { MsOHNMe }_{3}(-838.614773)-[\mathbf{2 8 b}(-2390.093585)+ \\
\left.\text { NMe }_{3}(-174.340524)\right]\end{array}$ & -0.03421 & -21.5 \\
\hline
\end{tabular}

\section{Energies $(\underline{M 062 x / 6-31 G * / 6-311+G * *)}$ and Cartesian Coordinates}

\section{$\mathrm{MsOHNMe}_{3}$}

SCF energy (6-31G*): -838.58370511 hartree

SCF energy $\left(6-311+\mathrm{G}^{* *}\right)$ : -838.75783883 hartree

zero-point correction: +0.189573 hartree

enthalpy correction: +0.206544 hartree

free energy correction: +0.140780 hartree

quasiharmonic free energy correction: +0.143066 hartree

imaginary frequency: none

$\begin{array}{llll}\mathrm{N} & 1.8878080 & -0.0476180 & 0.0691860 \\ \mathrm{C} & 1.9050070 & 1.2125270 & -0.7150750 \\ \mathrm{H} & 1.6868480 & 2.0417870 & -0.0405920 \\ \mathrm{H} & 2.8918380 & 1.3389660 & -1.1665740 \\ \mathrm{H} & 1.1254860 & 1.1414110 & -1.4734540 \\ \mathrm{C} & 2.0800600 & -1.2302750 & -0.8040950 \\ \mathrm{H} & 3.0581400 & -1.1624790 & -1.2867490 \\ \mathrm{H} & 2.0279200 & -2.1297020 & -0.1890680 \\ \mathrm{H} & 1.2728160 & -1.2329920 & -1.5360960 \\ \mathrm{C} & 2.8551920 & -0.0133980 & 1.1888860 \\ \mathrm{H} & 2.6159290 & 0.8357500 & 1.8297310 \\ \mathrm{H} & 2.7676340 & -0.9392840 & 1.7581490 \\ \mathrm{H} & 3.8672130 & 0.0884650 & 0.7902460 \\ \mathrm{H} & 0.9001650 & -0.1150580 & 0.5045120 \\ \mathrm{~S} & -1.4701230 & 0.1279540 & 0.0123840 \\ \mathrm{O} & -0.5005860 & -0.0895240 & 1.1493730 \\ \mathrm{O} & -2.1111120 & 1.4452400 & 0.0499290 \\ \mathrm{O} & -0.8218810 & -0.2210610 & -1.2728260 \\ \mathrm{C} & -2.7478230 & -1.0853640 & 0.2827670 \\ \mathrm{H} & -2.2977710 & -2.0781700 & 0.2560230 \\ \mathrm{H} & -3.2054350 & -0.8988620 & 1.2548770\end{array}$


$\mathrm{H} \quad-3.4894410 \quad-0.9819570 \quad-0.5101520$

$\mathrm{MsO}^{-}$

SCF energy (6-31G*): -663.69085366 hartree

SCF energy $\left(6-311+\mathrm{G}^{* *}\right)$ : -663.82024271 hartree

zero-point correction: +0.051671 hartree

enthalpy correction: +0.059327 hartree

free energy correction: +0.017015 hartree

quasiharmonic free energy correction: +0.017015 hartree

imaginary frequency: none

$\begin{array}{llll}\mathrm{O} & 0.5406990 & -1.0022360 & -1.0219520 \\ \mathrm{~S} & 0.1682190 & -0.0002090 & 0.0000770 \\ \mathrm{O} & 0.5538720 & 1.3834520 & -0.3521650 \\ \mathrm{O} & 0.5370380 & -0.3896250 & 1.3782970 \\ \mathrm{C} & -1.6312720 & 0.0076450 & -0.0037610 \\ \mathrm{H} & -1.9881510 & -0.9918590 & 0.2511480 \\ \mathrm{H} & -1.9846830 & 0.7292980 & 0.7348260 \\ \mathrm{H} & -1.9839090 & 0.2873040 & -0.9980770\end{array}$

$\mathrm{NMe}_{3}$

SCF energy (6-31G*): -174.3761922 hartree

SCF energy $\left(6-311+\mathrm{G}^{* *}\right):-174.42947092$ hartree

zero-point correction: +0.122444 hartree

enthalpy correction: +0.130613 hartree

free energy correction: +0.088947 hartree

quasiharmonic free energy correction: +0.088947 hartree imaginary frequency: none

$\begin{array}{llll}\mathrm{N} & -0.0002820 & -0.0002130 & -0.3949520 \\ \mathrm{C} & -1.3575130 & -0.2330710 & 0.0640460 \\ \mathrm{H} & -1.7162200 & -1.1945340 & -0.3157500 \\ \mathrm{H} & -1.4346330 & -0.2466780 & 1.1682530 \\ \mathrm{H} & -2.0160740 & 0.5543070 & -0.3148290 \\ \mathrm{C} & 0.4768610 & 1.2917440 & 0.0638570 \\ \mathrm{H} & 0.5048020 & 1.3649140 & 1.1680890 \\ \mathrm{H} & 1.4874920 & 1.4695850 & -0.3158350 \\ \mathrm{H} & -0.1769370 & 2.0834880 & -0.3142920 \\ \mathrm{C} & 0.8807180 & -1.0587030 & 0.0639710 \\ \mathrm{H} & 0.5295500 & -2.0230900 & -0.3153530 \\ \mathrm{H} & 1.8927630 & -0.8874740 & -0.3150610 \\ \mathrm{H} & 0.9308340 & -1.1188490 & 1.1682000\end{array}$

29a

SCF energy $\left(6-31 \mathrm{G}^{*}\right)$ : -1725.81532219 hartree

SCF energy $\left(6-311+\mathrm{G}^{* *}\right)$ : -1726.19240836 hartree 
zero-point correction: +0.408064 hartree

enthalpy correction: +0.445266 hartree

free energy correction: +0.335532 hartree

quasiharmonic free energy correction: +0.340794 hartree

imaginary frequency: none

$\begin{array}{llll}\mathrm{C} & 0.3032990 & -1.0040860 & -0.7582200 \\ \mathrm{C} & 1.4039080 & -1.4776440 & 0.1686270 \\ \mathrm{C} & 2.7389800 & -1.1351160 & -0.5029890 \\ \mathrm{C} & 1.7764920 & 0.7183770 & -1.6970240 \\ \mathrm{C} & 0.4077180 & 0.4964050 & -1.0244260 \\ \mathrm{H} & 1.3185490 & -0.9376720 & 1.1206630 \\ \mathrm{H} & 0.4085420 & -1.5435990 & -1.7156100 \\ \mathrm{H} & 1.7932030 & 0.1487660 & -2.6417590 \\ \mathrm{H} & 2.8406050 & -1.7635400 & -1.4021910 \\ \mathrm{C} & -0.7742310 & 0.8861850 & -1.8836270 \\ \mathrm{H} & -0.7296330 & 1.8112060 & -2.4539910 \\ \mathrm{O} & -0.9510730 & -1.3287990 & -0.1856680 \\ \mathrm{C} & -1.9859570 & -1.1559750 & -1.1258840 \\ \mathrm{H} & -1.9750120 & -1.9986390 & -1.8436990 \\ \mathrm{C} & -1.8709100 & 0.1297190 & -1.9093240 \\ \mathrm{H} & -2.7401540 & 0.3956520 & -2.5031800 \\ \mathrm{O} & 2.8032230 & 0.2368410 & -0.8345990 \\ \mathrm{O} & 1.3307700 & -2.8722260 & 0.3471380 \\ \mathrm{H} & 0.4144100 & -3.0616540 & 0.6048600 \\ \mathrm{~S} & 4.1843160 & -1.5276570 & 0.5167060 \\ \mathrm{C} & 3.8056880 & -0.4786060 & 1.9529000 \\ \mathrm{H} & 4.7448340 & -0.2975950 & 2.4779450 \\ \mathrm{H} & 3.1038960 & -0.9667020 & 2.6317120 \\ \mathrm{O} & 0.4351400 & 1.1887160 & 0.2072390 \\ \mathrm{Si} & -0.7110790 & 2.1518290 & 0.9638830 \\ \mathrm{C} & 0.1730100 & 2.6489870 & 2.5369030 \\ \mathrm{H} & 1.0997620 & 3.1846780 & 2.3076260 \\ \mathrm{H} & -0.4516380 & 3.2994220 & 3.1579630 \\ \mathrm{H} & 0.4353060 & 1.7655110 & 3.1282270 \\ \mathrm{C} & -2.2929510 & 1.2281630 & 1.3785990 \\ \mathrm{H} & -2.8140020 & 1.7396090 & 2.1962280 \\ \mathrm{H} & -2.9792040 & 1.1857180 & 0.5258410 \\ \mathrm{H} & -2.0726660 & 0.2045570 & 1.6983330 \\ \mathrm{C} & -1.1032520 & 3.6738860 & -0.0684750 \\ \mathrm{H} & -1.5897400 & 4.4320920 & 0.5559190 \\ \mathrm{H} & -0.1899740 & 4.1178450 & -0.4792960 \\ \mathrm{H} & -1.7780150 & 3.4463810 & -0.8992720 \\ \mathrm{C} & -3.3405100 & -1.2351980 & -0.4270550 \\ \mathrm{O} & -4.3470460 & -0.7599640 & -0.8956000 \\ & -3.2886620 & -1.9218400 & 0.7090090\end{array}$




$\begin{array}{llll}\mathrm{C} & -4.5302140 & -2.0044790 & 1.4180250 \\ \mathrm{H} & -4.8682720 & -1.0018290 & 1.6894340 \\ \mathrm{H} & -5.2885910 & -2.4875080 & 0.7992800 \\ \mathrm{H} & -4.3209950 & -2.5947150 & 2.3076440 \\ \mathrm{C} & 2.0958490 & 2.1776790 & -1.9529420 \\ \mathrm{H} & 3.0945820 & 2.2640520 & -2.3864280 \\ \mathrm{H} & 2.0734080 & 2.7247660 & -1.0072210 \\ \mathrm{H} & 1.3776730 & 2.6255060 & -2.6454040 \\ \mathrm{H} & 3.3988470 & 0.4729810 & 1.6040460\end{array}$

\section{TS B}

SCF energy (6-31G*): - 2564.33815299 hartree

SCF energy $\left(6-311+\mathrm{G}^{* *}\right):-2564.88422325$ hartree zero-point correction: +0.596720 hartree enthalpy correction: +0.650940 hartree free energy correction: +0.503513 hartree quasiharmonic free energy correction: +0.512718 hartree imaginary frequency: $-603.7 \mathrm{im}^{-1}$

$\begin{array}{llll}\mathrm{C} & -0.8736280 & -0.7696240 & 0.3349930 \\ \mathrm{C} & -2.1591120 & -1.3673230 & -0.2155680 \\ \mathrm{C} & -3.0730590 & -1.7019680 & 0.9761560 \\ \mathrm{C} & -2.1082220 & 0.0077070 & 2.3259560 \\ \mathrm{C} & -1.2394120 & 0.4872390 & 1.1331270 \\ \mathrm{H} & -2.6378200 & -0.6207310 & -0.8605800 \\ \mathrm{H} & -0.4038200 & -1.4942100 & 1.0198960 \\ \mathrm{H} & -1.5298310 & -0.7331280 & 2.9018330 \\ \mathrm{H} & -2.6155010 & -2.5425900 & 1.5232680 \\ \mathrm{C} & -0.0063930 & 1.2061450 & 1.6039070 \\ \mathrm{H} & -0.1639440 & 1.9424390 & 2.3877400 \\ \mathrm{O} & 0.0509070 & -0.4452460 & -0.6925800 \\ \mathrm{C} & 1.7257780 & 0.2585060 & -0.0002980 \\ \mathrm{H} & 2.0277120 & -0.7703870 & 0.1312200 \\ \mathrm{C} & 1.2325490 & 1.0993420 & 1.1297640 \\ \mathrm{H} & 2.0087000 & 1.7213270 & 1.5616560 \\ \mathrm{O} & -3.2919470 & -0.6048660 & 1.8212900 \\ \mathrm{O} & -1.8559010 & -2.5417350 & -0.9298940 \\ \mathrm{H} & -2.7037590 & -2.9383320 & -1.1954640 \\ \mathrm{~S} & -4.6877870 & -2.3212340 & 0.4115400 \\ \mathrm{C} & -5.3069610 & -0.7941380 & -0.3646980 \\ \mathrm{H} & -4.9941460 & 0.0561920 & 0.2445960 \\ \mathrm{H} & -6.3958940 & -0.8550460 & -0.3735280 \\ \mathrm{O} & -2.0752900 & 1.3423120 & 0.3849190 \\ \mathrm{Si} & -1.8618530 & 2.5747780 & -0.7356740 \\ \mathrm{C} & -3.5942640 & 3.2749500 & -0.8812690 \\ \mathrm{H} & -3.9419820 & 3.6607590 & 0.0825350\end{array}$




\begin{tabular}{|c|c|c|c|}
\hline $\mathrm{H}$ & -3.6293140 & 4.0948850 & -1.6068380 \\
\hline $\mathrm{H}$ & -4.3009980 & 2.5053280 & -1.2092750 \\
\hline $\mathrm{C}$ & -1.3426590 & 1.9065310 & -2.4099070 \\
\hline $\mathrm{H}$ & -1.1925650 & 2.7366600 & -3.1105310 \\
\hline $\mathrm{H}$ & -0.4305090 & 1.3068180 & -2.3723420 \\
\hline $\mathrm{H}$ & -2.1339880 & 1.2680830 & -2.8195150 \\
\hline $\mathrm{C}$ & -0.7105300 & 3.9241340 & -0.1259680 \\
\hline $\mathrm{H}$ & 0.3328160 & 3.6053060 & -0.0766190 \\
\hline $\mathrm{H}$ & -0.7783530 & 4.7812320 & -0.8072690 \\
\hline $\mathrm{H}$ & -1.0154780 & 4.2688150 & 0.8683890 \\
\hline $\mathrm{C}$ & 1.9494190 & 0.9153000 & -1.3504760 \\
\hline $\mathrm{O}$ & 2.1020580 & 0.3184290 & -2.3856830 \\
\hline $\mathrm{O}$ & 1.9060990 & 2.2382220 & -1.2404460 \\
\hline $\mathrm{C}$ & 2.1183360 & 2.9636470 & -2.4559900 \\
\hline $\mathrm{H}$ & 3.1373510 & 2.7984460 & -2.8110680 \\
\hline $\mathrm{H}$ & 1.4074020 & 2.6378230 & -3.2174890 \\
\hline $\mathrm{H}$ & 1.9619990 & 4.0103730 & -2.2014460 \\
\hline $\mathrm{C}$ & -2.5793420 & 1.1279840 & 3.2332290 \\
\hline $\mathrm{H}$ & -3.3226060 & 0.7304190 & 3.9276800 \\
\hline $\mathrm{H}$ & -3.0402990 & 1.9197620 & 2.6369840 \\
\hline $\mathrm{H}$ & -1.7550800 & 1.5432930 & 3.8170410 \\
\hline $\mathrm{H}$ & -4.9452550 & -0.6815220 & -1.3884810 \\
\hline $\mathrm{O}$ & 4.1195290 & 0.5806430 & 2.7337950 \\
\hline S & 4.2147770 & -0.1577140 & 1.4733590 \\
\hline $\mathrm{O}$ & 3.6536050 & 0.6517010 & 0.3009170 \\
\hline $\mathrm{O}$ & 3.6423500 & -1.5166940 & 1.4799770 \\
\hline $\mathrm{C}$ & 5.9393380 & -0.3008530 & 1.0504030 \\
\hline $\mathrm{H}$ & 0.5066480 & -1.3368210 & -1.0555830 \\
\hline $\mathrm{H}$ & 6.4311850 & -0.8666340 & 1.8429120 \\
\hline $\mathrm{H}$ & 6.0205480 & -0.8230990 & 0.0968710 \\
\hline $\mathrm{H}$ & 6.3615680 & 0.7017440 & 0.9793870 \\
\hline $\mathrm{N}$ & 1.2938660 & -2.6550550 & -1.4638050 \\
\hline $\mathrm{C}$ & 2.7570730 & -2.5622840 & -1.6117800 \\
\hline $\mathrm{H}$ & 3.0022750 & -1.7654810 & -2.3125160 \\
\hline $\mathrm{H}$ & 3.2164030 & -2.3507170 & -0.6417930 \\
\hline $\mathrm{H}$ & 3.1527500 & -3.5188160 & -1.9861640 \\
\hline $\mathrm{C}$ & 0.6964590 & -2.9545450 & -2.7708590 \\
\hline $\mathrm{H}$ & 0.9360570 & -2.1379020 & -3.4571170 \\
\hline $\mathrm{H}$ & 1.0952690 & -3.8959640 & -3.1779400 \\
\hline $\mathrm{H}$ & -0.3858130 & -3.0297600 & -2.6607910 \\
\hline $\mathrm{C}$ & 0.9927910 & -3.7155640 & -0.4909150 \\
\hline $\mathrm{H}$ & 1.4559430 & -3.4571430 & 0.4669550 \\
\hline $\mathrm{H}$ & -0.0868540 & -3.8047030 & -0.3677980 \\
\hline $\mathrm{H}$ & 1.4059880 & -4.6783030 & -0.8283070 \\
\hline
\end{tabular}

$28 b$ 
SCF energy (6-31G*): -2389.99196990 hartree

SCF energy $\left(6-311+\mathrm{G}^{* *}\right)$ : -2390.4899804 hartree zero-point correction: +0.473515 hartree enthalpy correction: +0.520302 hartree free energy correction: +0.388173 hartree quasiharmonic free energy correction: +0.396395 hartree imaginary frequency: none

$\begin{array}{llll}\mathrm{C} & -0.5547660 & -0.1056820 & -0.6082900 \\ \mathrm{C} & -1.6442470 & -1.0948730 & -0.9900430 \\ \mathrm{C} & -1.7594240 & -2.1404320 & 0.1189860 \\ \mathrm{C} & -1.0275970 & -0.5899930 & 1.7617110 \\ \mathrm{C} & -0.9000740 & 0.5619520 & 0.7227030 \\ \mathrm{H} & -2.5992390 & -0.5629800 & -1.0864730 \\ \mathrm{H} & 0.3561800 & -0.7017710 & -0.4755670 \\ \mathrm{H} & -0.0544670 & -1.1049040 & 1.8016300 \\ \mathrm{H} & -0.8236730 & -2.7182980 & 0.1627360 \\ \mathrm{C} & 0.1151470 & 1.5719250 & 1.2171330 \\ \mathrm{H} & -0.3400250 & 2.2881670 & 1.9003640 \\ \mathrm{O} & -0.3646430 & 0.8671580 & -1.6118740 \\ \mathrm{C} & 2.3074970 & 0.8515900 & 0.1348630 \\ \mathrm{H} & 1.8506780 & 0.7010530 & -0.8494290 \\ \mathrm{C} & 1.4241110 & 1.7080980 & 1.0052200 \\ \mathrm{H} & 1.9328720 & 2.5261890 & 1.5064490 \\ \mathrm{O} & -2.0322720 & -1.5111500 & 1.3487570 \\ \mathrm{O} & -1.2397990 & -1.6561290 & -2.2191640 \\ \mathrm{H} & -1.8806840 & -2.3474610 & -2.4530200 \\ \mathrm{~S} & -3.0693020 & -3.3510080 & -0.2253800 \\ \mathrm{C} & -4.5065860 & -2.2318110 & -0.1993570 \\ \mathrm{H} & -5.3840390 & -2.8379200 & 0.0300750 \\ \mathrm{H} & -4.6557760 & -1.7354770 & -1.1602910 \\ \mathrm{O} & -2.1629610 & 1.1895800 & 0.6570380 \\ \mathrm{Si} & -2.7130840 & 2.4732570 & -0.2818200 \\ \mathrm{C} & -3.4752220 & 1.8646880 & -1.8872580 \\ \mathrm{H} & -4.2504220 & 1.1152100 & -1.6892490 \\ \mathrm{H} & -3.9533400 & 2.7015690 & -2.4094540 \\ \mathrm{H} & -2.7312820 & 1.4246090 & -2.5559730 \\ \mathrm{C} & -1.3922350 & 3.7651460 & -0.6009920 \\ \mathrm{H} & -1.0691510 & 4.2499200 & 0.3260840 \\ \mathrm{H} & -0.5159580 & 3.3282760 & -1.0858120 \\ \mathrm{H} & -1.8010280 & 4.5420250 & -1.2577990 \\ \mathrm{C} & -4.0818480 & 3.2049600 & 0.7709210 \\ \mathrm{H} & -4.5666270 & 4.0443420 & 0.2607330 \\ \mathrm{H} & -4.8498310 & 2.4552820 & 0.9885510 \\ \mathrm{H} & -3.6908300 & 3.5702390 & 1.7261770 \\ \mathrm{C} & 3.6377810 & 1.5540410 & -0.1135850\end{array}$




$\begin{array}{llll}\mathrm{O} & 3.7101590 & 2.7380960 & -0.3244690 \\ \mathrm{O} & 4.6823870 & 0.7255650 & -0.1073920 \\ \mathrm{C} & 5.9410410 & 1.3392700 & -0.4151360 \\ \mathrm{H} & 5.9033110 & 1.7898940 & -1.4081080 \\ \mathrm{H} & 6.1725120 & 2.1070700 & 0.3249610 \\ \mathrm{H} & 6.6765950 & 0.5374820 & -0.3828830 \\ \mathrm{C} & -1.4356630 & -0.1090060 & 3.1400050 \\ \mathrm{H} & -1.6121830 & -0.9730920 & 3.7842490 \\ \mathrm{H} & -2.3553070 & 0.4771510 & 3.0699190 \\ \mathrm{H} & -0.6487760 & 0.5045310 & 3.5861020 \\ \mathrm{H} & -4.3615520 & -1.4928150 & 0.5911740 \\ \mathrm{O} & 1.7076050 & -2.6949510 & 0.2793670 \\ \mathrm{~S} & 2.7442630 & -1.7528750 & -0.1021060 \\ \mathrm{O} & 2.4995430 & -0.4203040 & 0.7942420 \\ \mathrm{O} & 2.8871950 & -1.3584790 & -1.4920910 \\ \mathrm{C} & 4.2868630 & -2.3148160 & 0.5654650 \\ \mathrm{H} & -0.3814540 & 0.3855490 & -2.4571090 \\ \mathrm{H} & 4.5177390 & -3.2605010 & 0.0728120 \\ \mathrm{H} & 5.0416180 & -1.5593840 & 0.3579670 \\ \mathrm{H} & 4.1515760 & -2.4628710 & 1.6370580\end{array}$

TS A

SCF energy (6-31G*): -3053.67576134 hartree SCF energy $\left(6-311+\mathrm{G}^{* *}\right)$ : -3054.30004196 hartree zero-point correction: +0.526006 hartree enthalpy correction: +0.580164 hartree free energy correction: +0.433998 hartree quasiharmonic free energy correction: +0.441640 hartree imaginary frequency: $-571.2 \mathrm{i} \mathrm{cm}-1$

$\begin{array}{llll}\mathrm{C} & -1.0593430 & -0.3865680 & 0.6141930 \\ \mathrm{C} & -2.1457070 & 0.2378760 & 1.4784530 \\ \mathrm{C} & -2.2541480 & 1.7360180 & 1.1619390 \\ \mathrm{C} & -1.4039940 & 1.4905430 & -1.0101860 \\ \mathrm{C} & -1.2999890 & -0.0480550 & -0.8744290 \\ \mathrm{H} & -3.1015580 & -0.2540850 & 1.2512200 \\ \mathrm{H} & -0.1206410 & 0.0731160 & 0.9468470 \\ \mathrm{H} & -0.4663490 & 1.9197830 & -0.6346310 \\ \mathrm{H} & -1.3277430 & 2.2303340 & 1.4954970 \\ \mathrm{C} & -0.2235810 & -0.5573570 & -1.8145790 \\ \mathrm{H} & -0.6428710 & -0.9355540 & -2.7455460 \\ \mathrm{O} & -1.0405040 & -1.7737020 & 0.8326620 \\ \mathrm{C} & 1.9198040 & -0.0800950 & -0.6024280 \\ \mathrm{H} & 1.5031460 & 0.4648860 & 0.2291180 \\ \mathrm{C} & 1.1108250 & -0.5596240 & -1.7281810 \\ \mathrm{H} & 1.6830800 & -0.9568820 & -2.5601850 \\ \mathrm{O} & -2.4801080 & 1.9623410 & -0.2040790\end{array}$




$\begin{array}{llll}\mathrm{O} & -1.7725130 & 0.0499700 & 2.8247150 \\ \mathrm{H} & -2.4044620 & 0.5550340 & 3.3626600 \\ \mathrm{~S} & -3.5954460 & 2.5227810 & 2.1103980 \\ \mathrm{C} & -5.0089600 & 1.6372630 & 1.3798990 \\ \mathrm{H} & -5.8947640 & 2.2554640 & 1.5344080 \\ \mathrm{H} & -5.1660060 & 0.6624550 & 1.8456840 \\ \mathrm{O} & -2.5452010 & -0.5774140 & -1.2874780 \\ \mathrm{Si} & -3.1182160 & -2.1533100 & -1.3396230 \\ \mathrm{C} & -3.9827190 & -2.6499290 & 0.2502340 \\ \mathrm{H} & -4.7641330 & -1.9234840 & 0.5042550 \\ \mathrm{H} & -4.4679410 & -3.6249520 & 0.1225100 \\ \mathrm{H} & -3.2773960 & -2.7140420 & 1.0816700 \\ \mathrm{C} & -1.8142770 & -3.4037510 & -1.8553620 \\ \mathrm{H} & -2.2141980 & -4.4163850 & -1.7262780 \\ \mathrm{H} & -1.5451670 & -3.2870480 & -2.9107920 \\ \mathrm{H} & -0.9039470 & -3.3229010 & -1.2567700 \\ \mathrm{C} & -4.4242330 & -2.0668690 & -2.6909480 \\ \mathrm{H} & -4.9075580 & -3.0396810 & -2.8360230 \\ \mathrm{H} & -5.2024250 & -1.3393090 & -2.4367110 \\ \mathrm{H} & -3.9822070 & -1.7627890 & -3.6457360 \\ \mathrm{C} & 3.4199330 & -0.2247080 & -0.5578160 \\ \mathrm{O} & 4.1054080 & 0.3509140 & 0.2526570 \\ \mathrm{O} & 3.8749730 & -1.0668460 & -1.4751060 \\ \mathrm{O} & 5.2690700 & -1.3707300 & -1.3627380 \\ \mathrm{C} & 3.6601990 & 3.5124290 & -0.2023790 \\ \mathrm{H} & 5.8632860 & -0.4644370 & -1.4984450 \\ \mathrm{H} & 5.4566360 & -1.8016260 & -0.3779890 \\ \mathrm{H} & 5.4756470 & -2.0897010 & -2.1535560 \\ \mathrm{C} & -1.6641840 & 1.9665110 & -2.4236500 \\ \mathrm{H} & -1.7986620 & 3.0503030 & -2.4144800 \\ \mathrm{H} & -2.5641750 & 1.4906890 & -2.8225220 \\ \mathrm{H} & -0.8088720 & 1.7327040 & -3.0632560 \\ \mathrm{H} & -4.8306240 & 1.5194840 & 0.3090960 \\ \mathrm{O} & 1.7313960 & -3.3888140 & 2.2183470 \\ \mathrm{~S} & 2.4228800 & -2.2330820 & 1.6481580 \\ \mathrm{O} & 1.6794130 & -1.7933930 & 0.3680090 \\ \mathrm{O} & 3.8585600 & -2.3766730 & 1.3962340 \\ \mathrm{C} & 2.1810520 & -0.8543720 & 2.7528350 \\ \mathrm{H} & -0.1259310 & -2.0728140 & 0.6520300 \\ \mathrm{H} & 2.6843100 & -1.0827730 & 3.6935540 \\ \mathrm{H} & 1.6145110 & 0.0403670 & 2.2995820 \\ \mathrm{H} & 1.3046560 & 4.0140030 & -1.2472850 \\ \mathrm{H} & 2.2087020 & 1.7347940 & -1.4695020 \\ \mathrm{H} & & & \\ \mathrm{H} & & & \\ \mathrm{H} & & & \end{array}$




$\begin{array}{llll}\mathrm{H} & 4.1316050 & 3.7921530 & -1.1453540 \\ \mathrm{H} & 3.5832070 & 4.3777170 & 0.4574400 \\ \mathrm{H} & 4.2028420 & 2.6976880 & 0.2773590\end{array}$

28a

SCF energy (6-31G*): -2389.99143958 hartree

SCF energy $\left(6-311+\mathrm{G}^{* *}\right)$ : -2390.48794832 hartree zero-point correction: +0.473661 hartree enthalpy correction: +0.520110 hartree free energy correction: +0.389688 hartree quasiharmonic free energy correction: +0.397018 hartree imaginary frequency: none

$\begin{array}{llll}\mathrm{C} & -0.8968150 & -0.3273690 & -0.7460800 \\ \mathrm{C} & -2.2726740 & -0.8578880 & -1.1113710 \\ \mathrm{C} & -2.7120630 & -1.8827290 & -0.0687370 \\ \mathrm{C} & -1.4238100 & -0.8233290 & 1.6184260 \\ \mathrm{C} & -0.9298130 & 0.2944450 & 0.6513990 \\ \mathrm{H} & -2.9808030 & -0.0192030 & -1.1140150 \\ \mathrm{H} & -0.2107210 & -1.1887910 & -0.7337330 \\ \mathrm{H} & -0.6925840 & -1.6448980 & 1.5678060 \\ \mathrm{H} & -2.0404270 & -2.7558780 & -0.1214920 \\ \mathrm{C} & 0.3923510 & 0.8251220 & 1.1691230 \\ \mathrm{H} & 0.2369220 & 1.4582550 & 2.0422450 \\ \mathrm{O} & -0.4749570 & 0.6287510 & -1.6971390 \\ \mathrm{C} & 2.2107800 & -0.1221260 & -0.3740060 \\ \mathrm{H} & 1.5110800 & -0.0515510 & -1.2128480 \\ \mathrm{C} & 1.6612180 & 0.6375910 & 0.8060380 \\ \mathrm{H} & 2.4233290 & 1.1133930 & 1.4180360 \\ \mathrm{O} & -2.7023480 & -1.3063590 & 1.2106110 \\ \mathrm{O} & -2.1377390 & -1.4258700 & -2.3969230 \\ \mathrm{H} & -3.0063120 & -1.7734340 & -2.6579990 \\ \mathrm{~S} & -4.3706440 & -2.5384190 & -0.4169980 \\ \mathrm{C} & -5.3350600 & -1.0116510 & -0.1800320 \\ \mathrm{H} & -6.3639760 & -1.3112210 & 0.0238920 \\ \mathrm{H} & -5.3172190 & -0.3750980 & -1.0664320 \\ \mathrm{O} & -1.8919890 & 1.3240270 & 0.7080820 \\ \mathrm{Si} & -1.9702220 & 2.8189170 & -0.0638290 \\ \mathrm{C} & -2.9965940 & 2.7002580 & -1.6337750 \\ \mathrm{H} & -3.9734220 & 2.2479170 & -1.4260800 \\ \mathrm{H} & -3.1782920 & 3.7039440 & -2.0350850 \\ \mathrm{H} & -2.4992340 & 2.1103050 & -2.4078380 \\ \mathrm{C} & -0.2893470 & 3.5716840 & -0.4064480 \\ \mathrm{H} & 0.2647690 & 3.7646600 & 0.5175460 \\ \mathrm{H} & 0.3185640 & 2.9236890 & -1.0421240 \\ \mathrm{H} & -0.4283220 & 4.5313940 & -0.9184450\end{array}$




$\begin{array}{llll}\mathrm{C} & -2.9075140 & 3.8814890 & 1.1648190 \\ \mathrm{H} & -3.0620170 & 4.8924700 & 0.7722270 \\ \mathrm{H} & -3.8905220 & 3.4518900 & 1.3845690 \\ \mathrm{H} & -2.3595230 & 3.9657250 & 2.1090340 \\ \mathrm{C} & 2.4870050 & -1.6141060 & -0.1576430 \\ \mathrm{O} & 3.3713200 & -2.2207970 & -0.7080890 \\ \mathrm{O} & 1.5715570 & -2.1735840 & 0.6306110 \\ \mathrm{C} & 1.6973550 & -3.5918840 & 0.8016030 \\ \mathrm{H} & 1.6293550 & -4.0931690 & -0.1655630 \\ \mathrm{H} & 2.6557740 & -3.8245430 & 1.2680120 \\ \mathrm{H} & 0.8716890 & -3.8835880 & 1.4481470 \\ \mathrm{C} & -1.5926490 & -0.3529000 & 3.0500060 \\ \mathrm{H} & -2.0792290 & -1.1417000 & 3.6279330 \\ \mathrm{H} & -2.2166130 & 0.5439680 & 3.0751820 \\ \mathrm{H} & -0.6245550 & -0.1347590 & 3.5064020 \\ \mathrm{H} & -4.9362110 & -0.4745470 & 0.6824170 \\ \mathrm{O} & 5.1634680 & 1.9099740 & 0.0871470 \\ \mathrm{~S} & 4.7496580 & 0.5257810 & -0.0162640 \\ \mathrm{O} & 3.3856690 & 0.5100440 & -0.9031560 \\ \mathrm{O} & 4.5192000 & -0.2521380 & 1.1902470 \\ \mathrm{C} & 5.8445910 & -0.3359850 & -1.1135060 \\ \mathrm{H} & -0.7416920 & 0.2800010 & -2.5656080 \\ \mathrm{H} & 6.8227970 & -0.3606970 & -0.6306940 \\ \mathrm{H} & 5.4398620 & -1.3363820 & -1.2614140 \\ \mathrm{H} & 5.8911990 & 0.2230920 & -2.0482830\end{array}$

TS C

SCF energy (6-31G*): -2564.34628816 hartree

$\mathrm{SCF}$ energy $\left(6-311+\mathrm{G}^{* *}\right)$ : -2564.89429504 hartree zero-point correction: +0.596292 hartree enthalpy correction: +0.650832 hartree free energy correction: +0.502736 hartree quasiharmonic free energy correction: +0.511784 hartree imaginary frequency: - $624.05 \mathrm{i} \mathrm{cm}^{-1}$

$\begin{array}{llll}\mathrm{C} & 0.9998110 & -0.5044480 & -0.6425030 \\ \mathrm{C} & 2.1896330 & -1.2273180 & -0.0302760 \\ \mathrm{C} & 3.3592460 & -1.1498690 & -1.0174960 \\ \mathrm{C} & 2.5906650 & 0.9105090 & -1.9403590 \\ \mathrm{C} & 1.3946310 & 0.9420850 & -0.9634810 \\ \mathrm{H} & 2.4640240 & -0.7257090 & 0.9082160 \\ \mathrm{H} & 0.7878700 & -1.0080610 & -1.6053070 \\ \mathrm{H} & 2.2681040 & 0.3981890 & -2.8623640 \\ \mathrm{H} & 3.0949300 & -1.7373930 & -1.9123130 \\ \mathrm{C} & 0.2007400 & 1.6495400 & -1.5603260 \\ \mathrm{H} & 0.4239020 & 2.5204920 & -2.1737650 \\ \mathrm{O} & -0.1084100 & -0.5106210 & 0.1814130\end{array}$




$\begin{array}{llll}\mathrm{C} & -1.7578640 & 0.2865370 & -0.6074450 \\ \mathrm{H} & -1.9910120 & 0.4522170 & 0.4342170 \\ \mathrm{C} & -1.0956520 & 1.3618000 & -1.4147230 \\ \mathrm{H} & -1.8113000 & 1.9948380 & -1.9291390 \\ \mathrm{O} & 3.6678210 & 0.1760710 & -1.3529220 \\ \mathrm{O} & 1.8194720 & -2.5770410 & 0.1962320 \\ \mathrm{H} & 2.6425880 & -3.0614390 & 0.3857440 \\ \mathrm{~S} & 4.8594870 & -1.9465520 & -0.3599510 \\ \mathrm{C} & 5.1779610 & -0.8238600 & 1.0384030 \\ \mathrm{H} & 4.9401450 & 0.1945770 & 0.7255500 \\ \mathrm{H} & 6.2405090 & -0.8927820 & 1.2749080 \\ \mathrm{O} & 1.8585190 & 1.5743260 & 0.2165190 \\ \mathrm{Si} & 1.1690080 & 2.7561440 & 1.1822190 \\ \mathrm{C} & 0.9400790 & 4.3702580 & 0.2475350 \\ \mathrm{H} & 0.0925450 & 4.3283800 & -0.4428080 \\ \mathrm{H} & 0.7493960 & 5.1833640 & 0.9575790 \\ \mathrm{H} & 1.8371840 & 4.6312120 & -0.3242560 \\ \mathrm{C} & 2.4683480 & 2.9731300 & 2.5172530 \\ \mathrm{H} & 3.4297560 & 3.2639030 & 2.0815910 \\ \mathrm{H} & 2.1745520 & 3.7436880 & 3.2378960 \\ \mathrm{H} & 2.6195110 & 2.0370940 & 3.0659720 \\ \mathrm{C} & -0.4480550 & 2.2163980 & 1.9655830 \\ \mathrm{H} & -1.3093190 & 2.3874000 & 1.3121990 \\ \mathrm{H} & -0.4008380 & 1.1454700 & 2.1858420 \\ \mathrm{H} & -0.6233370 & 2.7609050 & 2.8999010 \\ \mathrm{H} & -1.9445710 & -1.1157490 & -1.1089660 \\ \mathrm{O} & -2.5045000 & -1.9961890 & -0.4871370 \\ \mathrm{O} & -1.4490700 & -1.2805220 & -2.3324060 \\ \mathrm{C} & -1.5190760 & -2.6147180 & -2.8429410 \\ \mathrm{H} & -1.1002870 & -2.5671170 & -3.8462460 \\ \mathrm{H} & -0.9299100 & -3.2841480 & -2.2112020 \\ \mathrm{H} & -2.5551960 & -2.9558510 & -2.8711240 \\ \mathrm{C} & 3.1687490 & 2.2742100 & -2.2663920 \\ \mathrm{H} & 4.0544710 & 2.1511880 & -2.8939820 \\ \mathrm{H} & 3.4598530 & 2.7764350 & -1.3404590 \\ \mathrm{H} & 2.4518900 & 2.8996760 & -2.8038550 \\ \mathrm{H} & 4.5944820 & -1.0946890 & 1.9200130 \\ \mathrm{H} & -3.9894370 & 0.5045130 & 1.2796370 \\ \mathrm{H} & -4.5722100 & 0.7059190 & -0.0535440 \\ \mathrm{H} & -5.454030570 & 0.7330420 & -1.1304130 \\ \mathrm{H} & -6.8548200 & -0.2269840 \\ \mathrm{H} & -0.7563440 & -0.4569730 \\ \mathrm{H} & -1.5930 & -1.5864030 & 1.1417270 \\ \mathrm{H} & -0.8481310 & 0.2712640 \\ \mathrm{H} & -0.6375180 & -1.4614730 \\ \mathrm{H} & -1.6128070 & -0.4067270\end{array}$




$\begin{array}{llll}\mathrm{N} & -0.6574370 & -2.1741610 & 2.0406350 \\ \mathrm{C} & -1.9161720 & -1.5847280 & 2.5611450 \\ \mathrm{H} & -2.6885600 & -1.6772430 & 1.7991140 \\ \mathrm{H} & -2.2016630 & -2.1052470 & 3.4796380 \\ \mathrm{H} & -1.7532140 & -0.5252710 & 2.7682190 \\ \mathrm{C} & 0.4533930 & -1.9933250 & 2.9992900 \\ \mathrm{H} & 0.2045160 & -2.4841390 & 3.9441520 \\ \mathrm{H} & 1.3536780 & -2.4274490 & 2.5650940 \\ \mathrm{H} & 0.6004200 & -0.9233870 & 3.1616260 \\ \mathrm{C} & -0.8340870 & -3.5946340 & 1.6676070 \\ \mathrm{H} & -1.6410670 & -3.6558610 & 0.9375820 \\ \mathrm{H} & 0.0977480 & -3.9452880 & 1.2243840 \\ \mathrm{H} & -1.0808250 & -4.1738690 & 2.5618790\end{array}$

\section{9b}

SCF energy (6-31G*): -1725.81737506 hartree

$\mathrm{SCF}$ energy $\left(6-311+\mathrm{G}^{* *}\right)$ : -1726.19412413 hartree zero-point correction: +0.408206 hartree enthalpy correction: +0.445479 hartree free energy correction: +0.335441 hartree quasiharmonic free energy correction: +0.340573 hartree imaginary frequency: none

$\begin{array}{llll}\mathrm{C} & 0.3900290 & -0.7251590 & -0.0165510 \\ \mathrm{C} & -0.6658310 & -1.5944390 & -0.6565830 \\ \mathrm{C} & -1.5680870 & -2.1232340 & 0.4674650 \\ \mathrm{C} & -1.0541340 & -0.2763480 & 1.9141370 \\ \mathrm{C} & -0.1998630 & 0.3994780 & 0.8240300 \\ \mathrm{H} & -1.2542910 & -0.9827780 & -1.3540840 \\ \mathrm{H} & 0.9984670 & -1.3729370 & 0.6347670 \\ \mathrm{H} & -0.3953680 & -0.9181110 & 2.5236720 \\ \mathrm{H} & -0.9809750 & -2.8461440 & 1.0555670 \\ \mathrm{C} & 0.9707930 & 1.1937770 & 1.3664730 \\ \mathrm{H} & 0.8279800 & 1.8081720 & 2.2524900 \\ \mathrm{O} & 1.2139990 & -0.1884330 & -1.0404130 \\ \mathrm{C} & 2.3905140 & 0.3549320 & -0.5074740 \\ \mathrm{H} & 2.8076420 & 1.0246390 & -1.2709960 \\ \mathrm{C} & 2.1593000 & 1.1478260 & 0.7621620 \\ \mathrm{H} & 3.0162860 & 1.6943220 & 1.1458530 \\ \mathrm{O} & -2.0504270 & -1.0778870 & 1.2872660 \\ \mathrm{O} & -0.0607600 & -2.6900330 & -1.3024340 \\ \mathrm{H} & 0.8207240 & -2.3959630 & -1.5888330 \\ \mathrm{~S} & -2.9984140 & -3.0558850 & -0.1425540 \\ \mathrm{C} & -3.8355720 & -1.7251290 & -1.0561210 \\ \mathrm{H} & -4.8815370 & -2.0178130 & -1.1614430 \\ \mathrm{H} & -3.4036620 & -1.5818400 & -2.0482040\end{array}$




$\begin{array}{llll}\mathrm{O} & -1.0773730 & 1.1682900 & 0.0301380 \\ \mathrm{Si} & -0.9307770 & 2.7080600 & -0.6163170 \\ \mathrm{C} & -2.5553320 & 2.9199220 & -1.5205490 \\ \mathrm{H} & -3.3981530 & 2.8319500 & -0.8273870 \\ \mathrm{H} & -2.6169420 & 3.8989740 & -2.0071510 \\ \mathrm{H} & -2.6740270 & 2.1506160 & -2.2902910 \\ \mathrm{C} & 0.5055130 & 2.8693670 & -1.8148020 \\ \mathrm{H} & 0.3459460 & 3.7321750 & -2.4714810 \\ \mathrm{H} & 1.4551160 & 3.0214580 & -1.2914510 \\ \mathrm{H} & 0.5934780 & 1.9734440 & -2.4372230 \\ \mathrm{C} & -0.7757420 & 3.9970540 & 0.7441740 \\ \mathrm{H} & -0.9505370 & 4.9966730 & 0.3297570 \\ \mathrm{H} & -1.5164570 & 3.8301970 & 1.5335740 \\ \mathrm{H} & 0.2181790 & 3.9980720 & 1.2020240 \\ \mathrm{C} & 3.4127480 & -0.7706530 & -0.2983000 \\ \mathrm{O} & 3.2034970 & -1.9313500 & -0.5474680 \\ \mathrm{O} & 4.5634960 & -0.2909330 & 0.1752680 \\ \mathrm{C} & 5.5943280 & -1.2663830 & 0.3804810 \\ \mathrm{H} & 5.2677980 & -2.0073070 & 1.1123710 \\ \mathrm{H} & 5.8285260 & -1.7667050 & -0.5608760 \\ \mathrm{H} & 6.4555970 & -0.7126700 & 0.7482880 \\ \mathrm{C} & -1.7958390 & 0.7088300 & 2.7952520 \\ \mathrm{H} & -2.4270450 & 0.1648150 & 3.5012530 \\ \mathrm{H} & -2.4288430 & 1.3467420 & 2.1735630 \\ \mathrm{H} & -1.1018450 & 1.3351730 & 3.3629440 \\ \mathrm{H} & -3.7769600 & -0.8001140 & -0.4790190\end{array}$


${ }^{1} \mathrm{H}-\mathrm{NMR}\left(500 \mathrm{MHz}, \mathrm{CDCl}_{3}\right.$ )

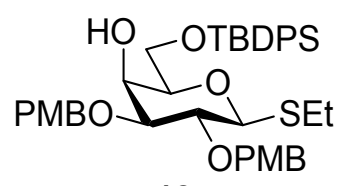

12

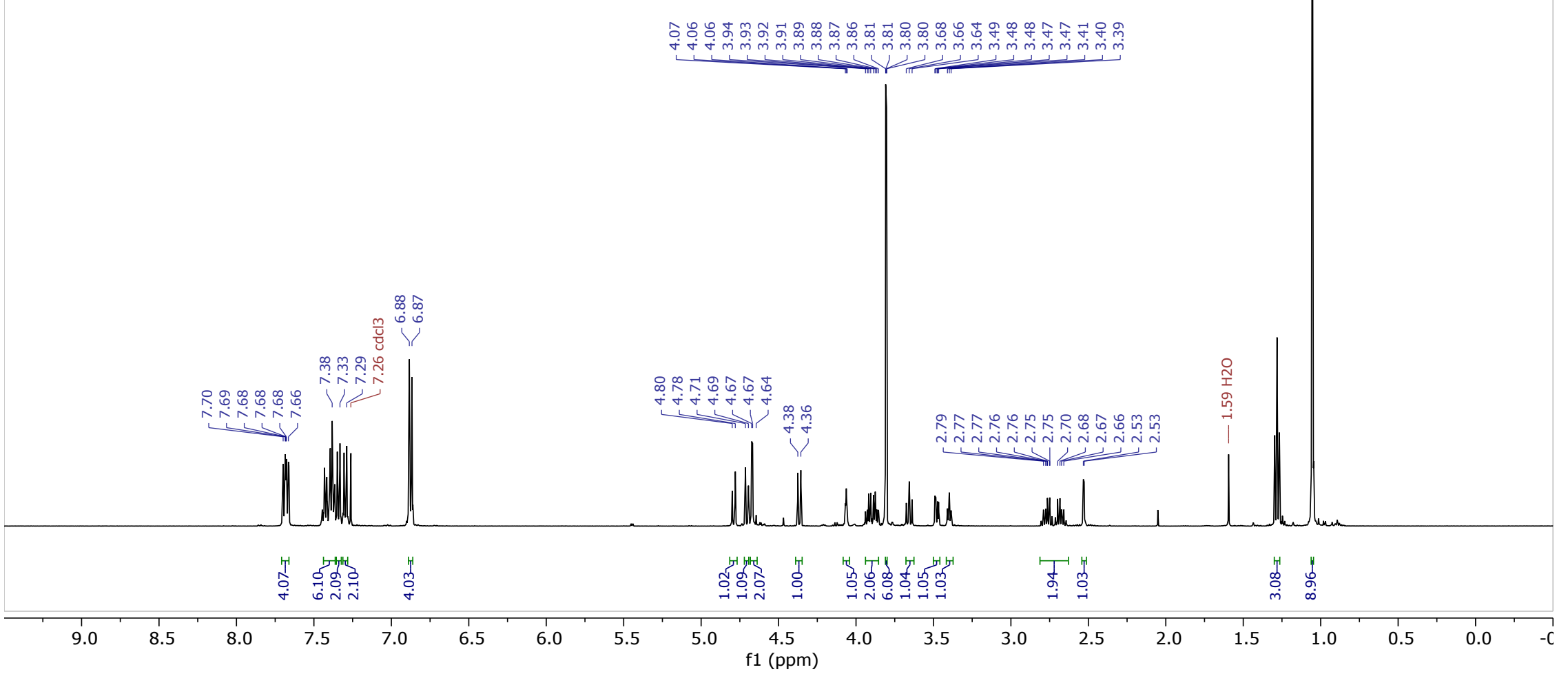


${ }^{13} \mathrm{C}\left\{{ }^{1} \mathrm{H}\right\}-\mathrm{NMR}\left(126 \mathrm{MHz}, \mathrm{CDCl}_{3}\right)$

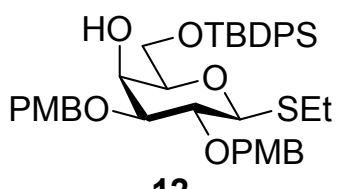

12

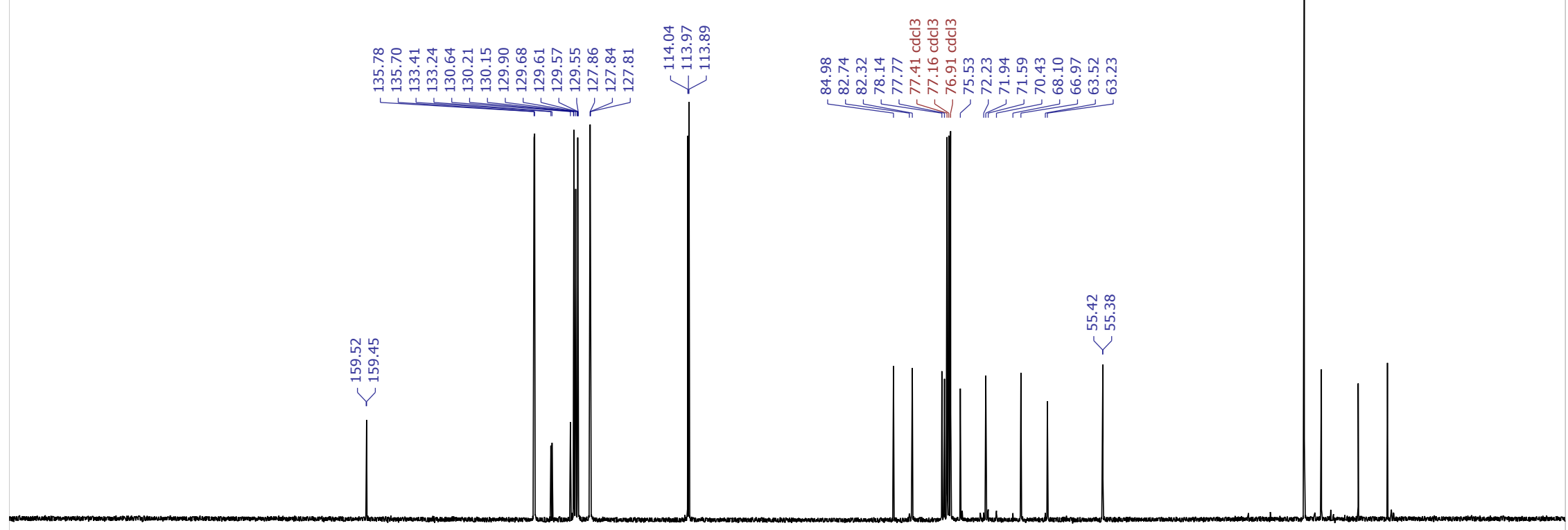

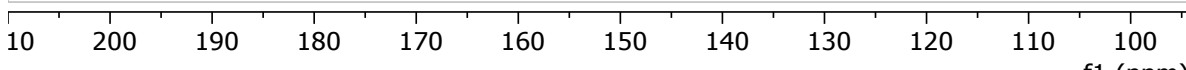

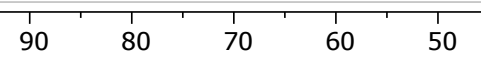

40

30

20 
${ }^{1} \mathrm{H}-\mathrm{NMR}\left(500 \mathrm{MHz}, \mathrm{CDCl}_{3}\right.$ )

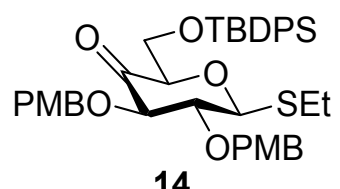

14

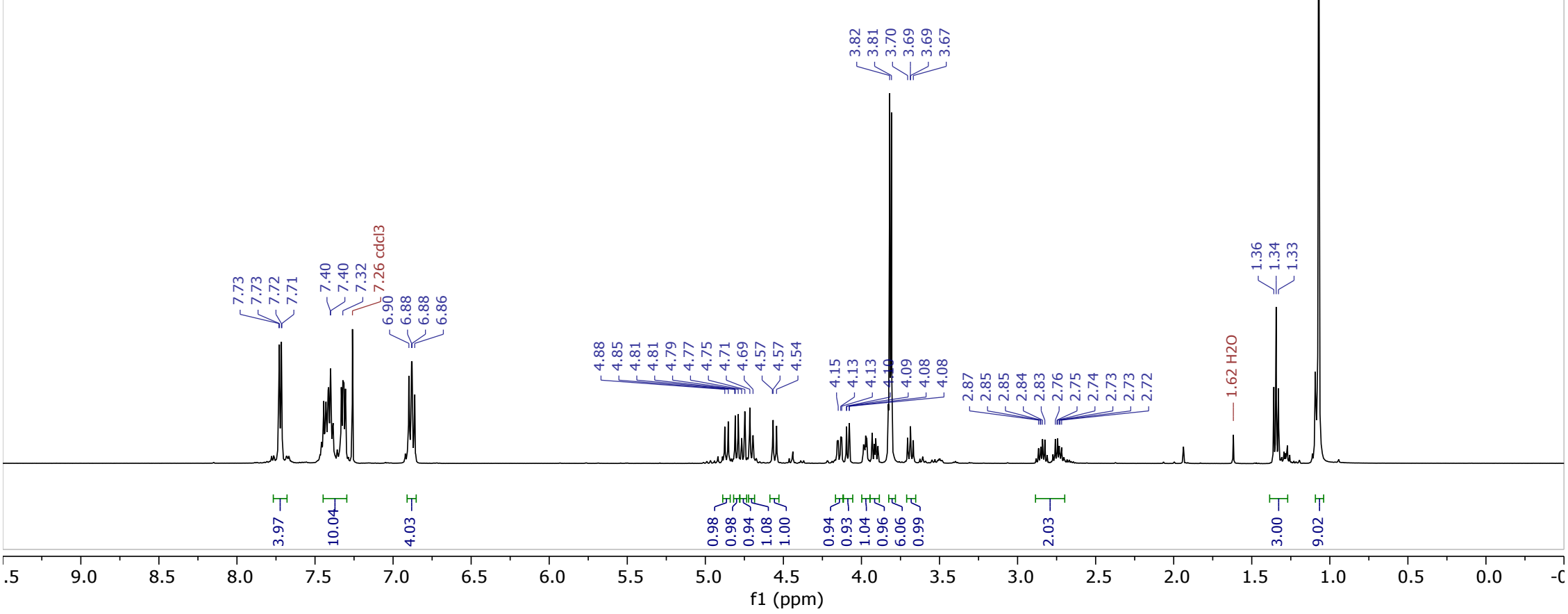


${ }^{13} \mathrm{C}\left\{{ }^{1} \mathrm{H}\right\}-\mathrm{NMR}\left(126 \mathrm{MHz}, \mathrm{CDCl}_{3}\right.$ )
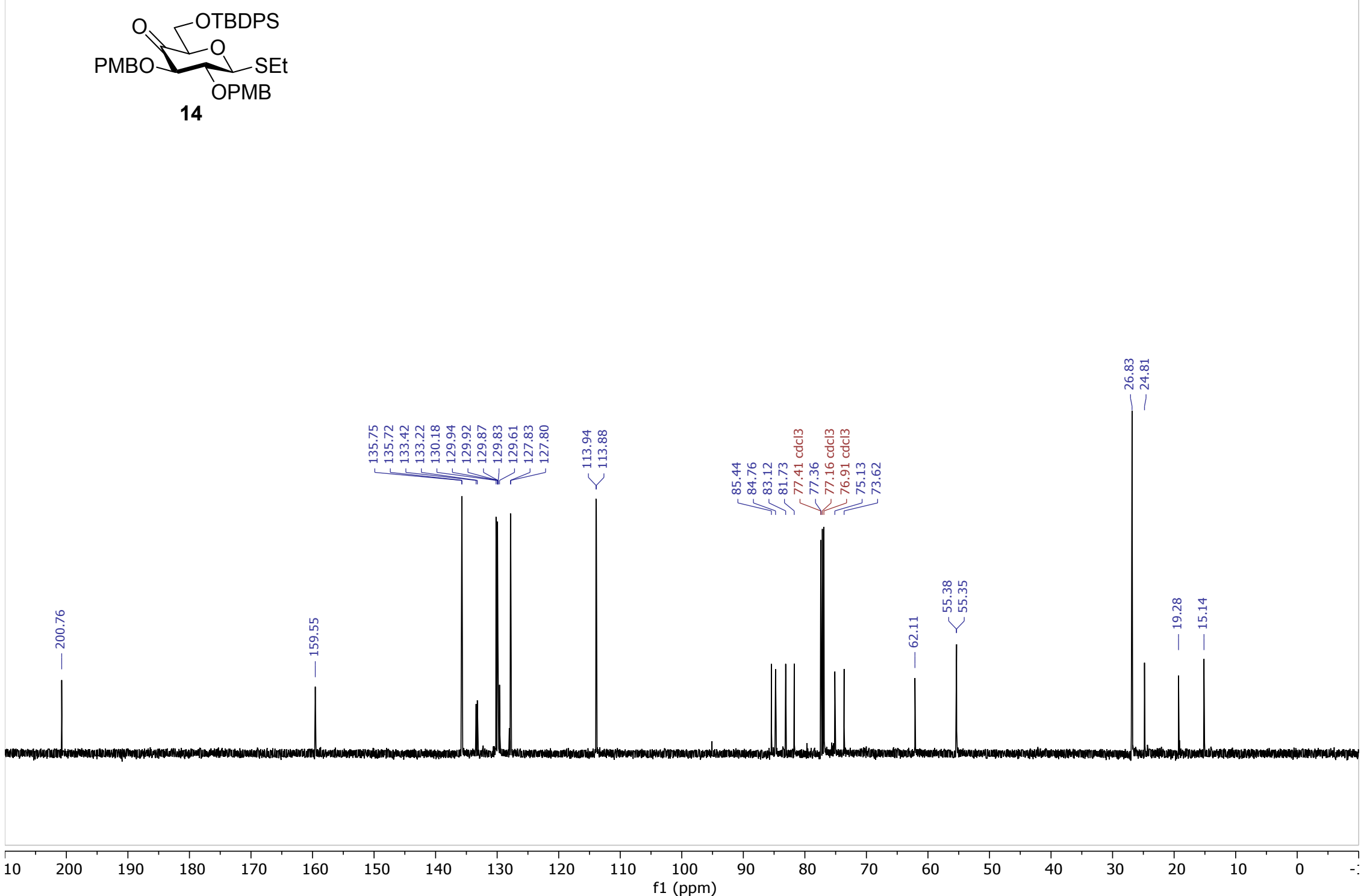
${ }^{1} \mathrm{H}-\mathrm{NMR}\left(500 \mathrm{MHz}, \mathrm{CDCl}_{3}\right)$

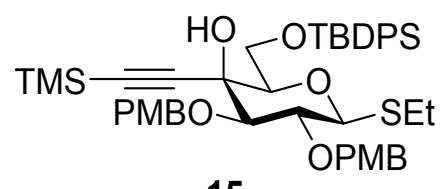

15

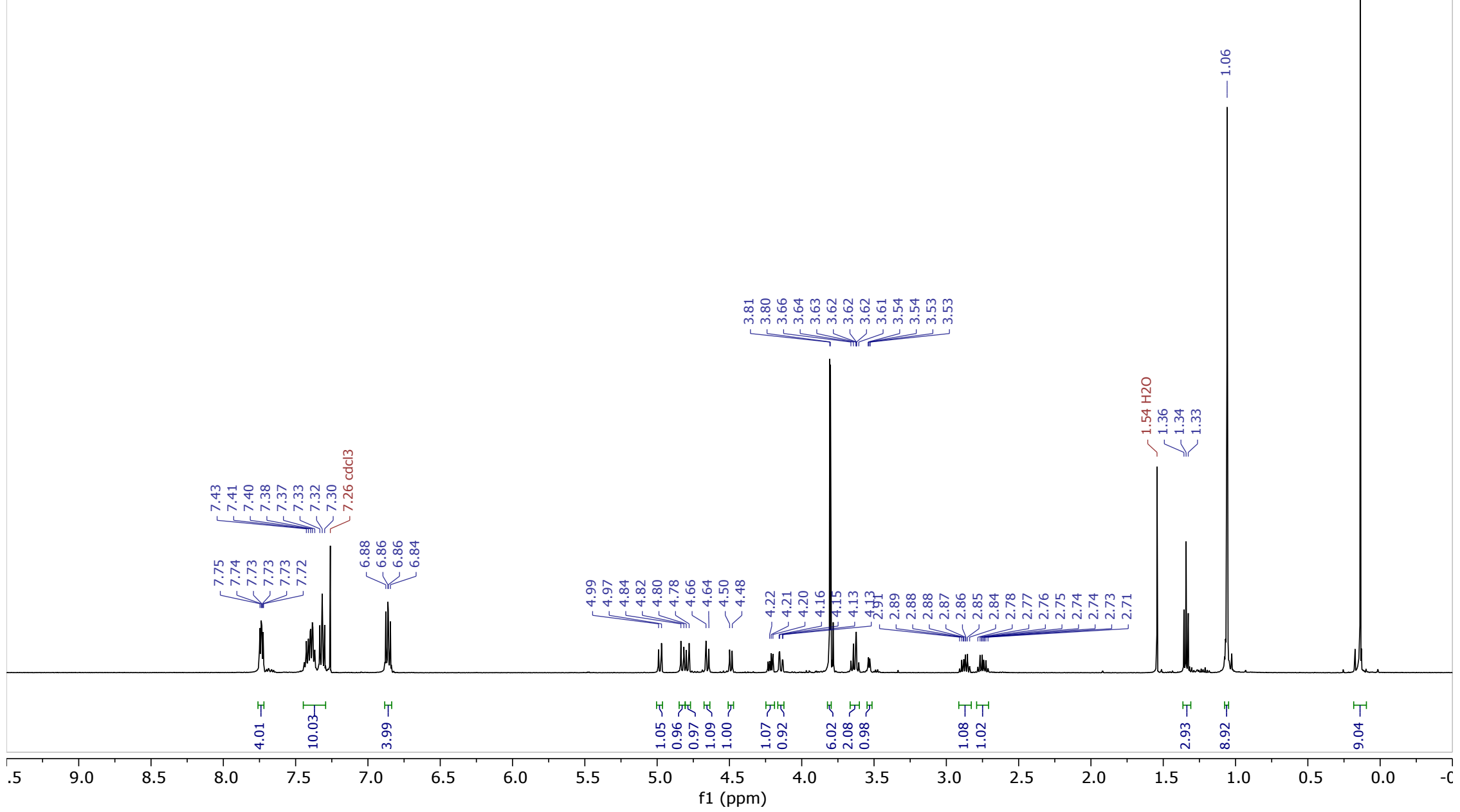


${ }^{13} \mathrm{C}\left\{{ }^{1} \mathrm{H}\right\}-\mathrm{NMR}\left(126 \mathrm{MHz}, \mathrm{CDCl}_{3}\right)$

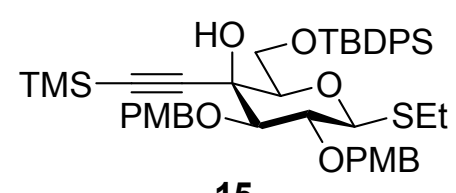

15

PPMB
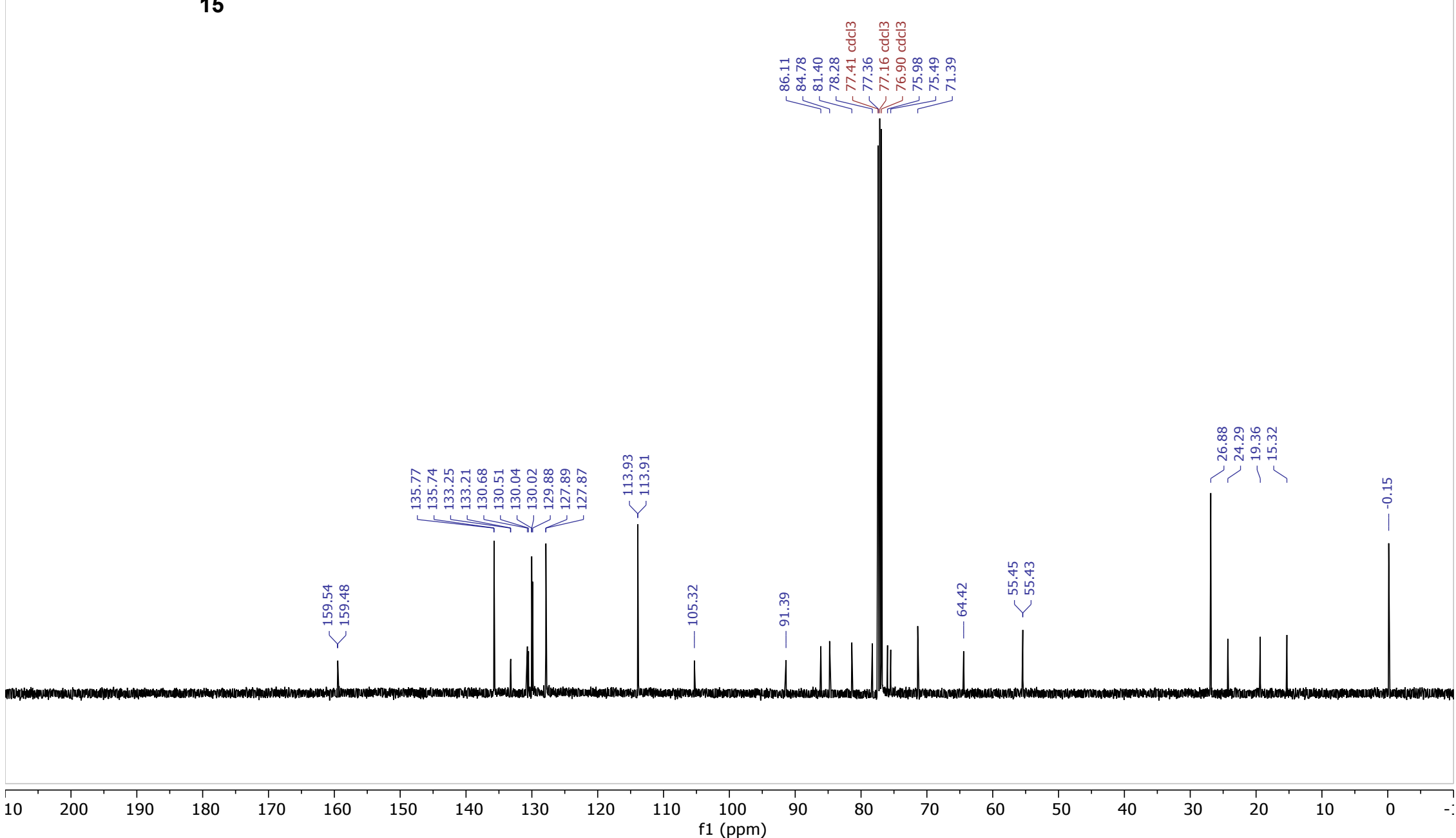


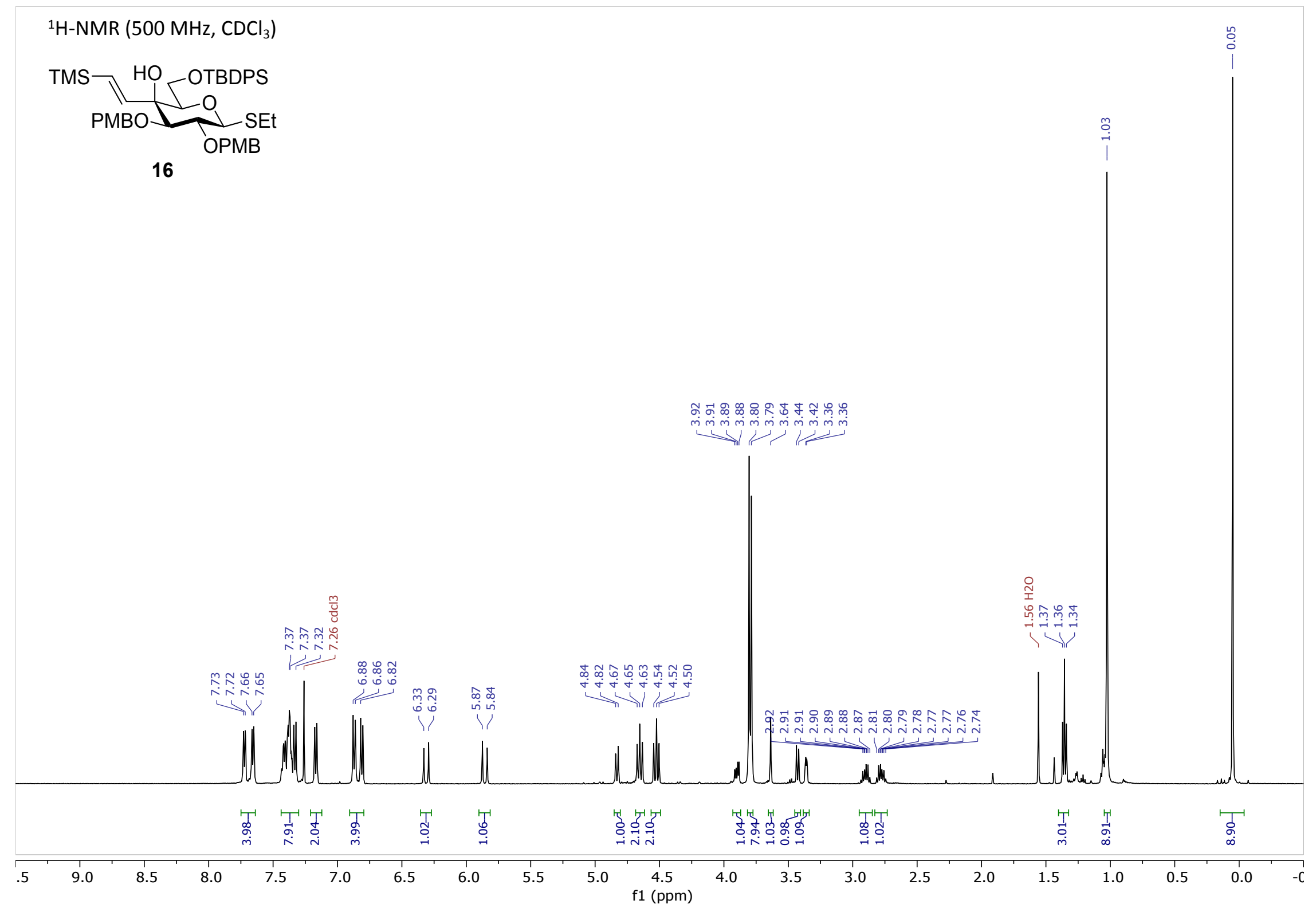




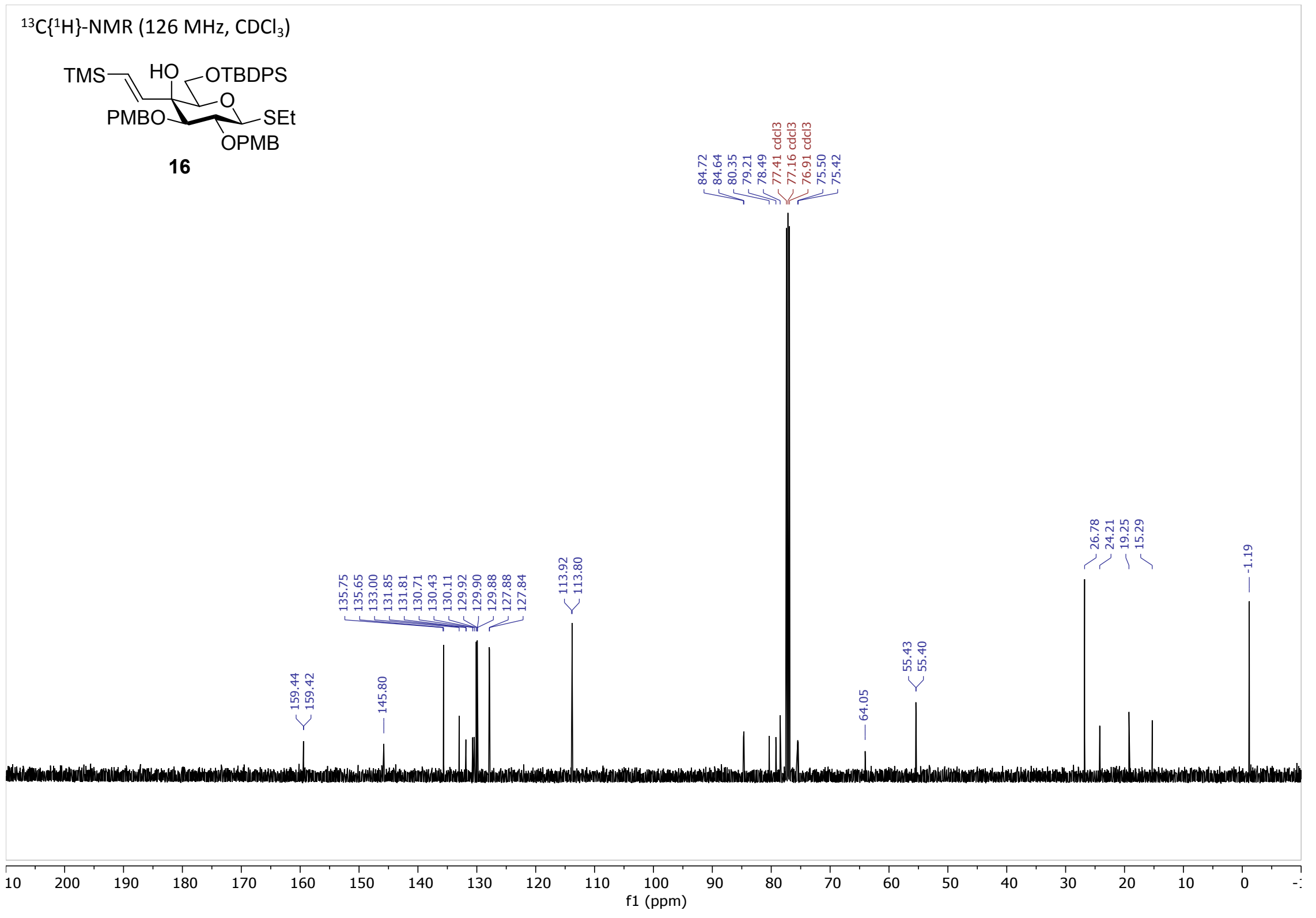


${ }^{1} \mathrm{H}-\mathrm{NMR}\left(500 \mathrm{MHz}, \mathrm{CDCl}_{3}\right.$ )

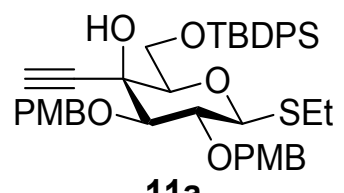

$11 \mathrm{a}$

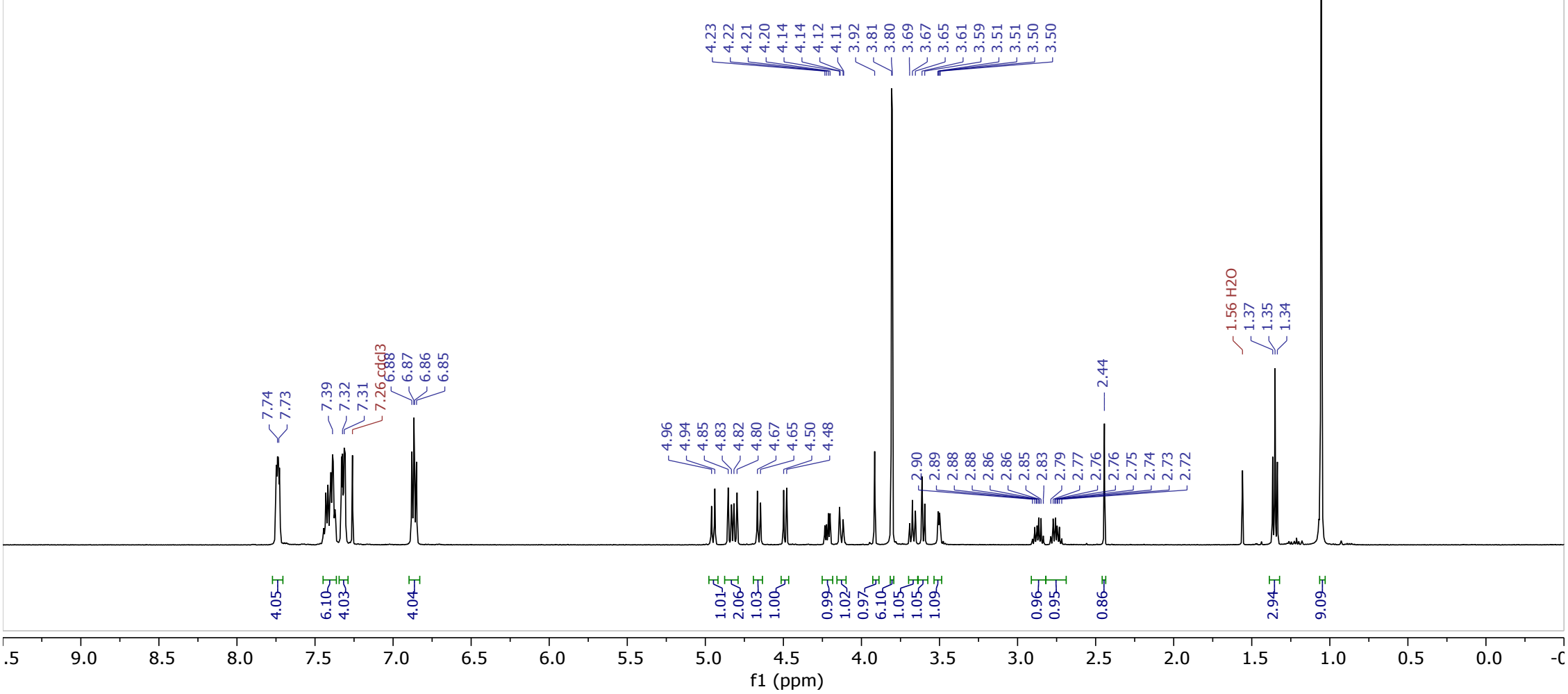


${ }^{13} \mathrm{C}\left\{\mathrm{H}^{1}\right\}-N M R\left(126 \mathrm{MHz}, \mathrm{CDCl}_{3}\right)$
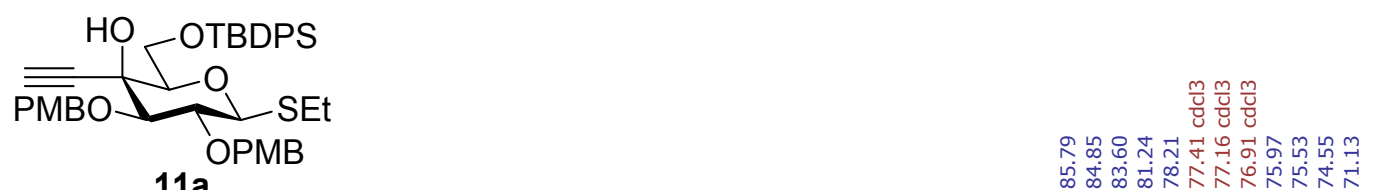

$11 \mathrm{a}$

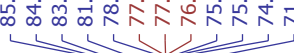

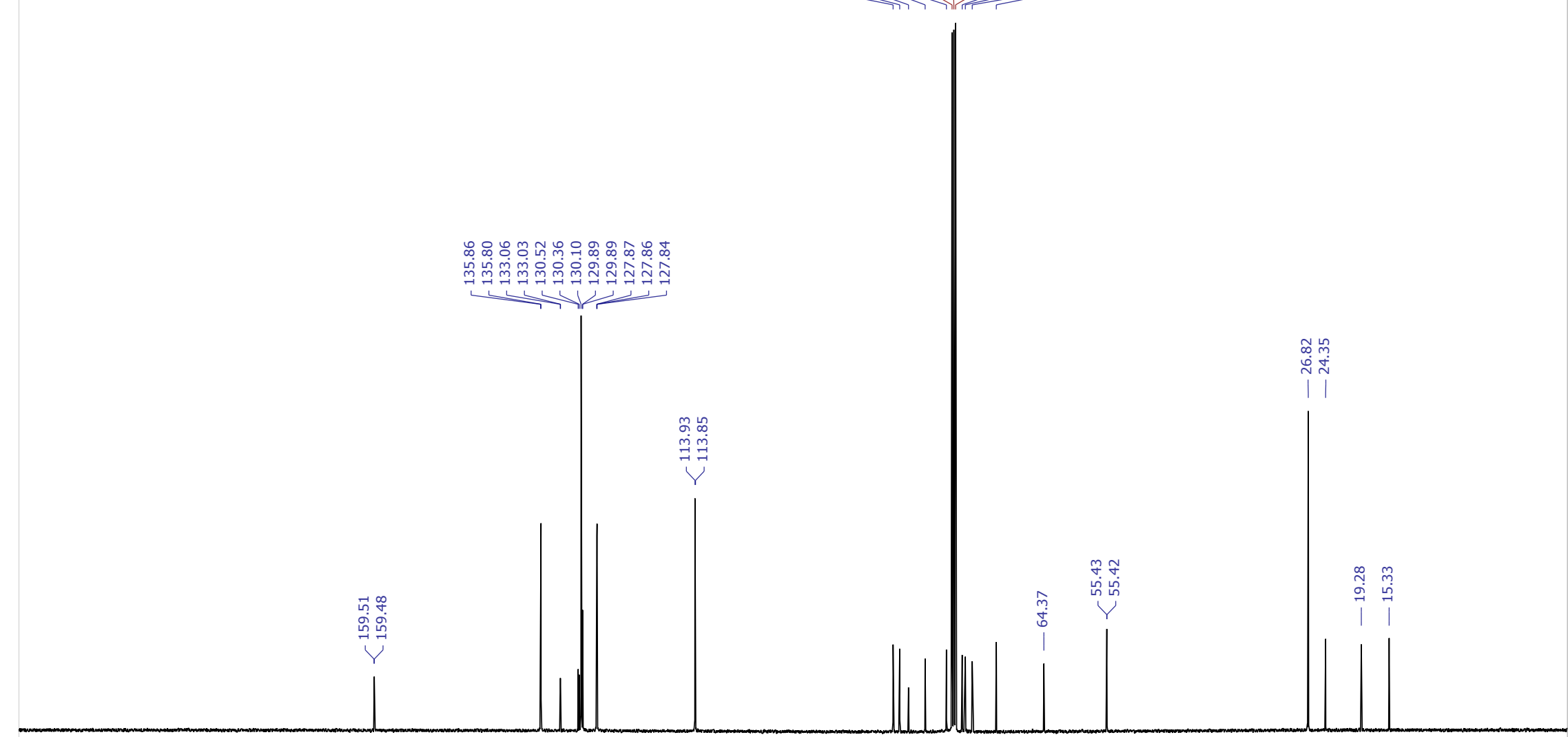$$
10
$$ 
${ }^{1} \mathrm{H}-\mathrm{NMR}\left(500 \mathrm{MHz}, \mathrm{CDCl}_{3}\right.$ )

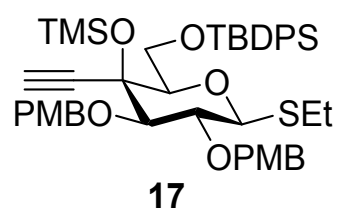

17

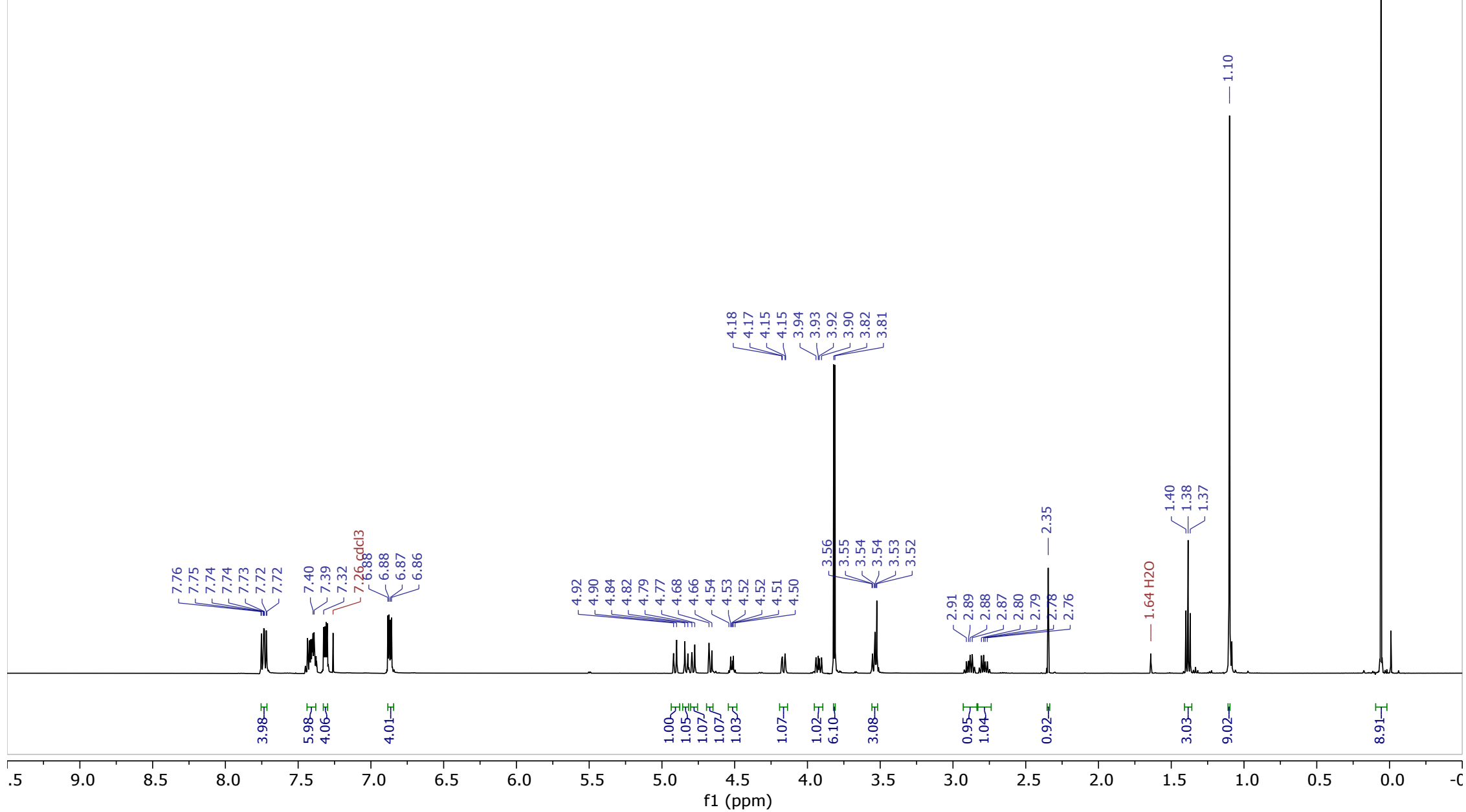


${ }^{13} \mathrm{C}\left\{{ }^{1} \mathrm{H}\right\}-\mathrm{NMR}\left(126 \mathrm{MHz}, \mathrm{CDCl}_{3}\right)$

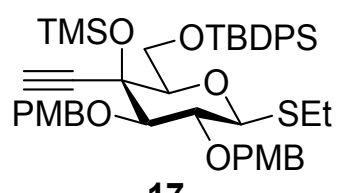

17

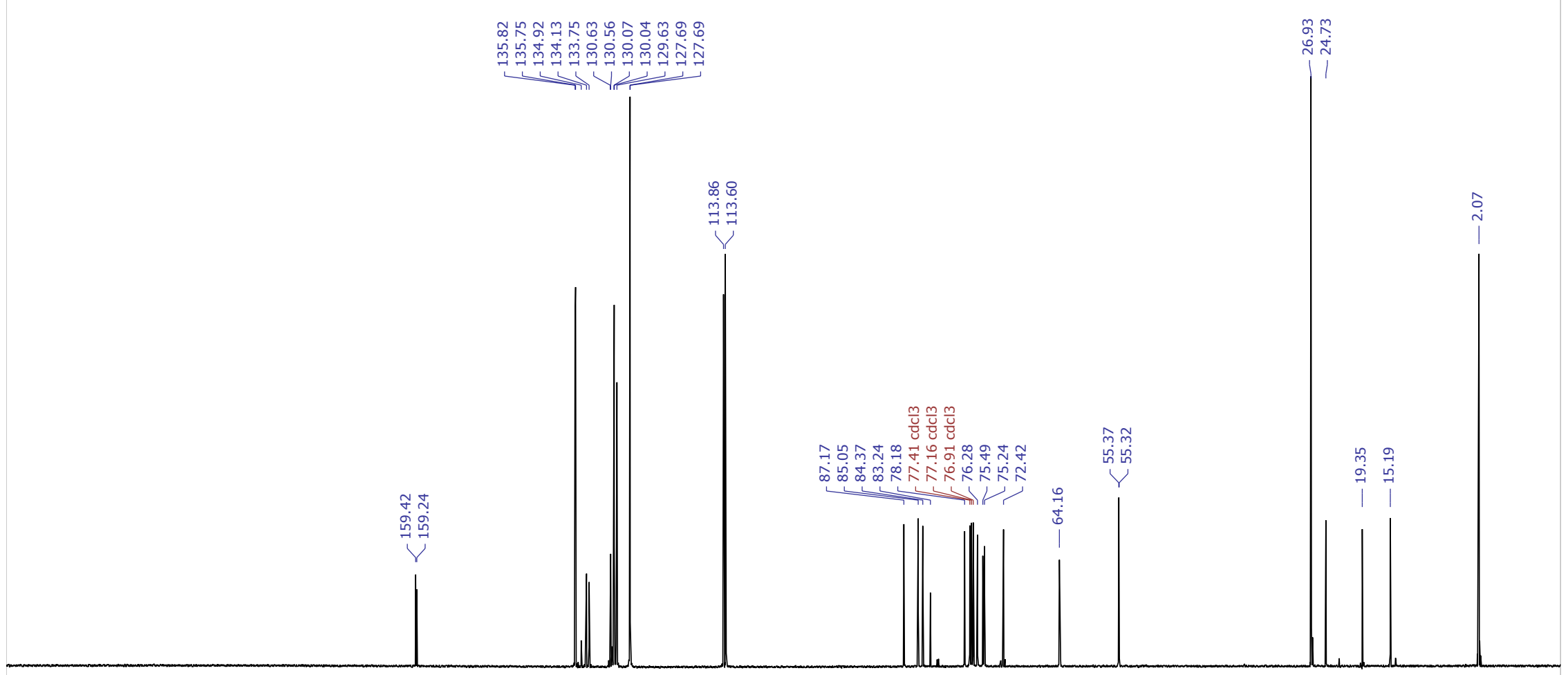

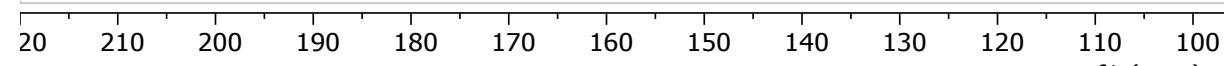
f1 (ppm)

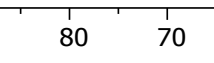

$60 \quad 50$

$40 \quad 30$

$20 \quad 10$ 0 
${ }^{1} \mathrm{H}-\mathrm{NMR}\left(500 \mathrm{MHz}, \mathrm{CDCl}_{3}\right)$

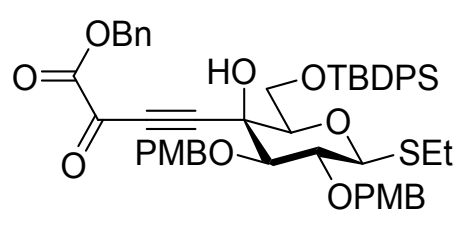

18

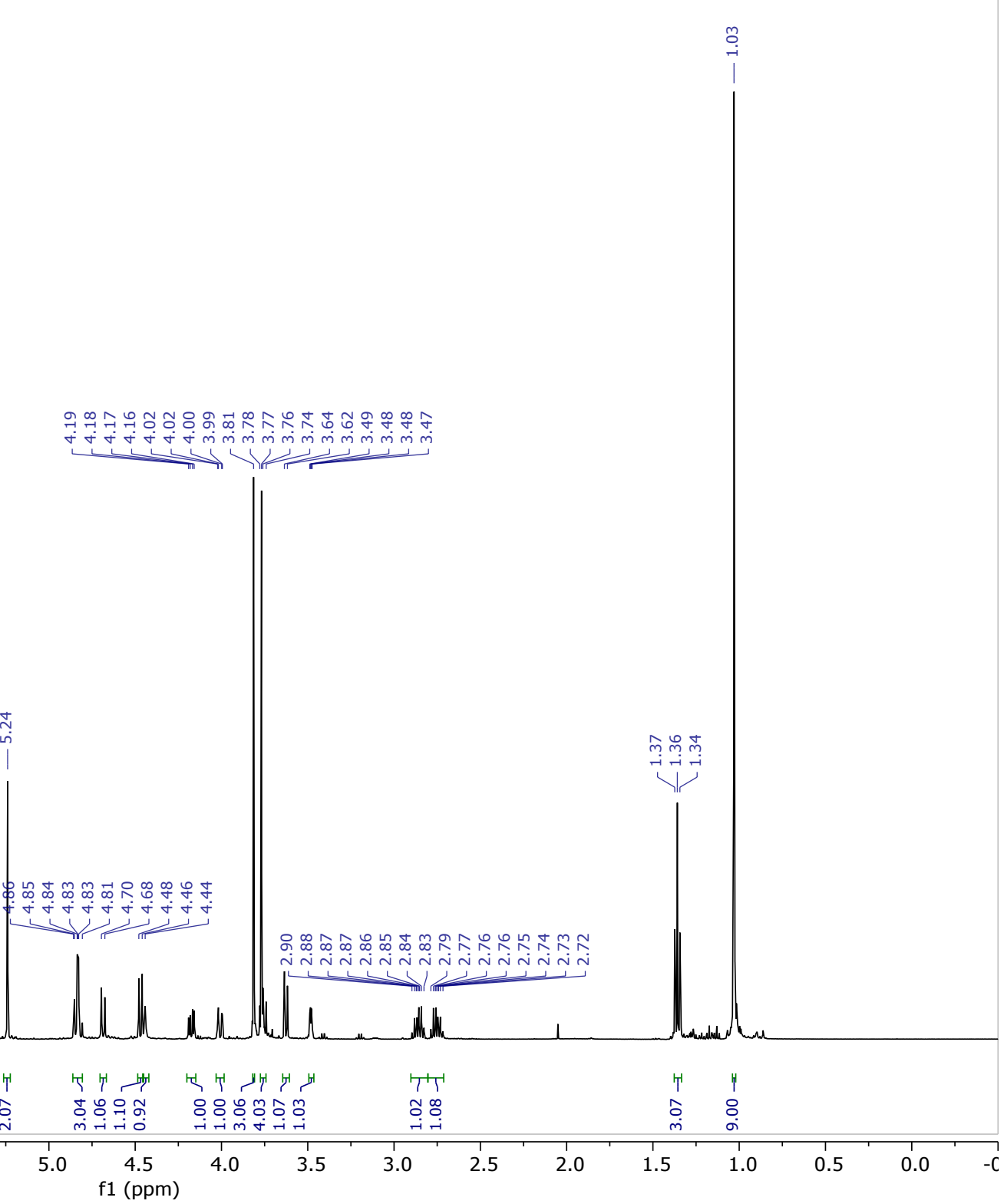



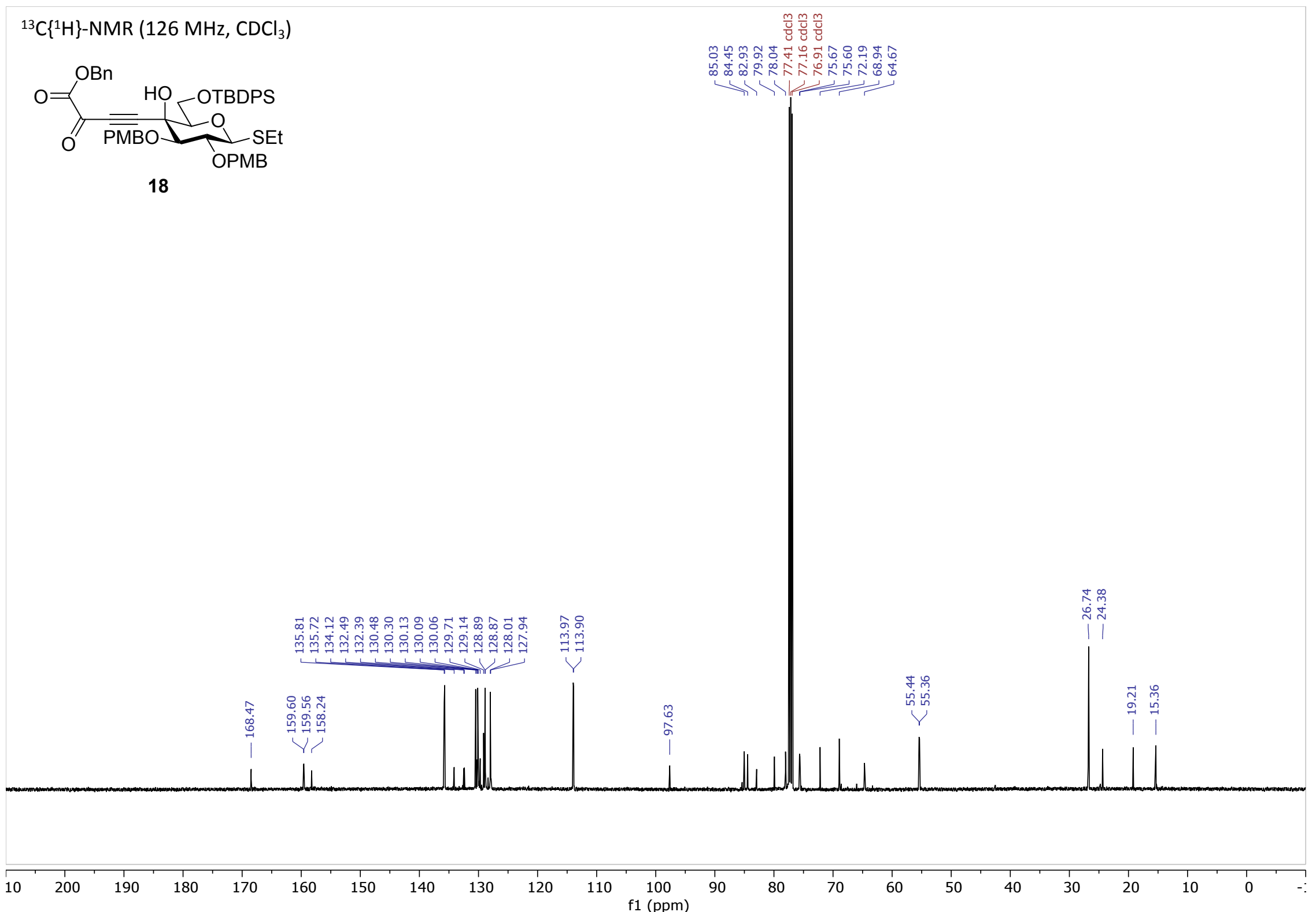
${ }^{1} \mathrm{H}-\mathrm{NMR}\left(500 \mathrm{MHz}, \mathrm{CDCl}_{3}\right)$

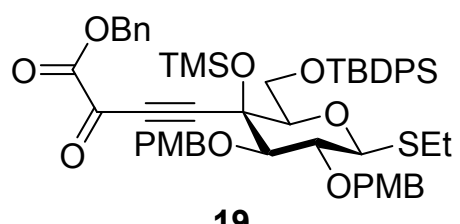

19

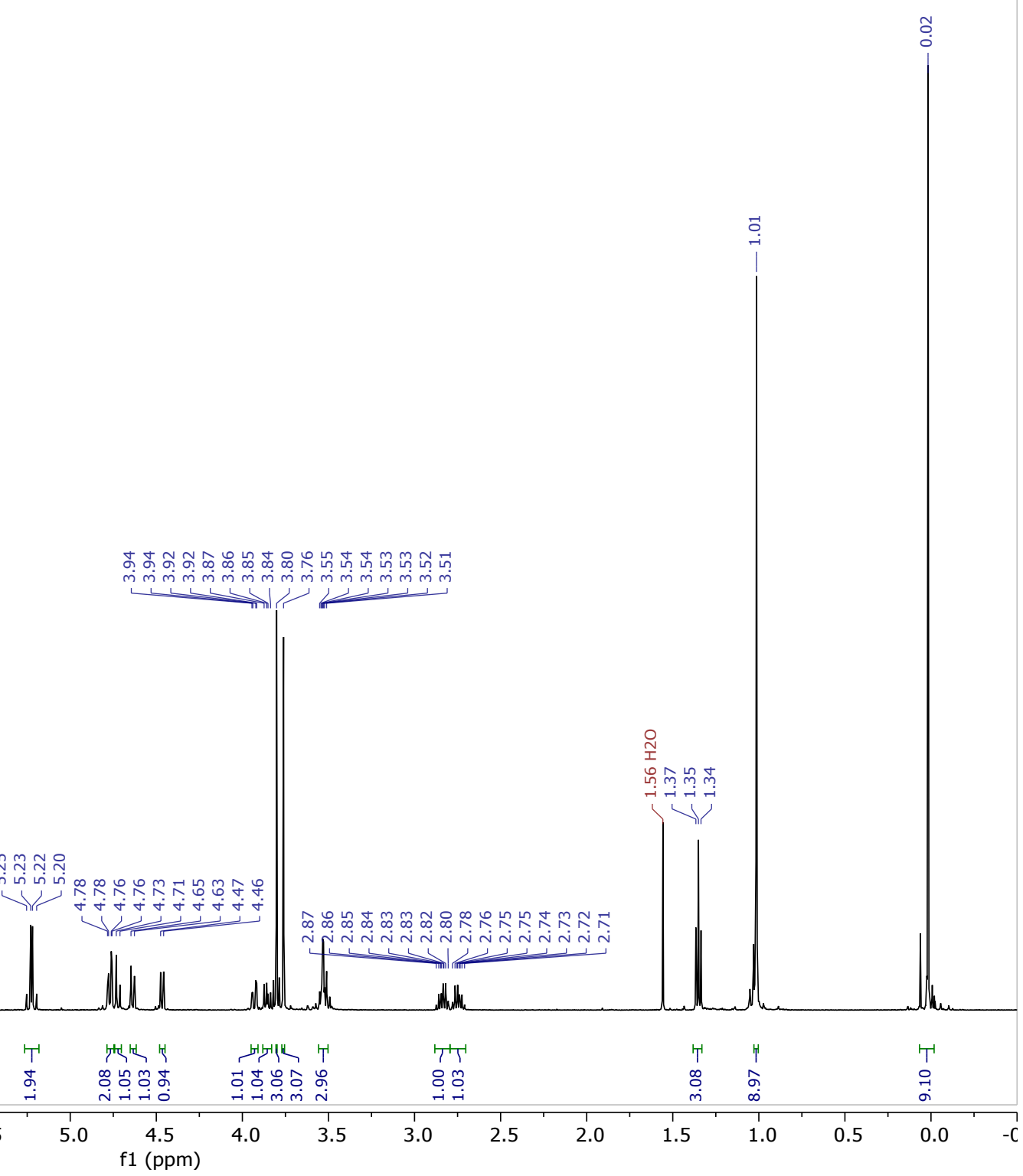


${ }^{13} \mathrm{C}\left\{{ }^{1} \mathrm{H}\right\}-\mathrm{NMR}\left(126 \mathrm{MHz}, \mathrm{CDCl}_{3}\right)$

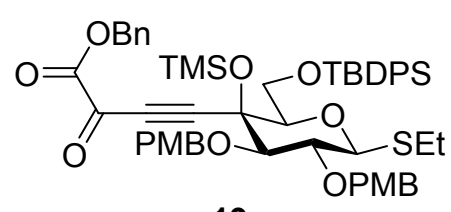

19
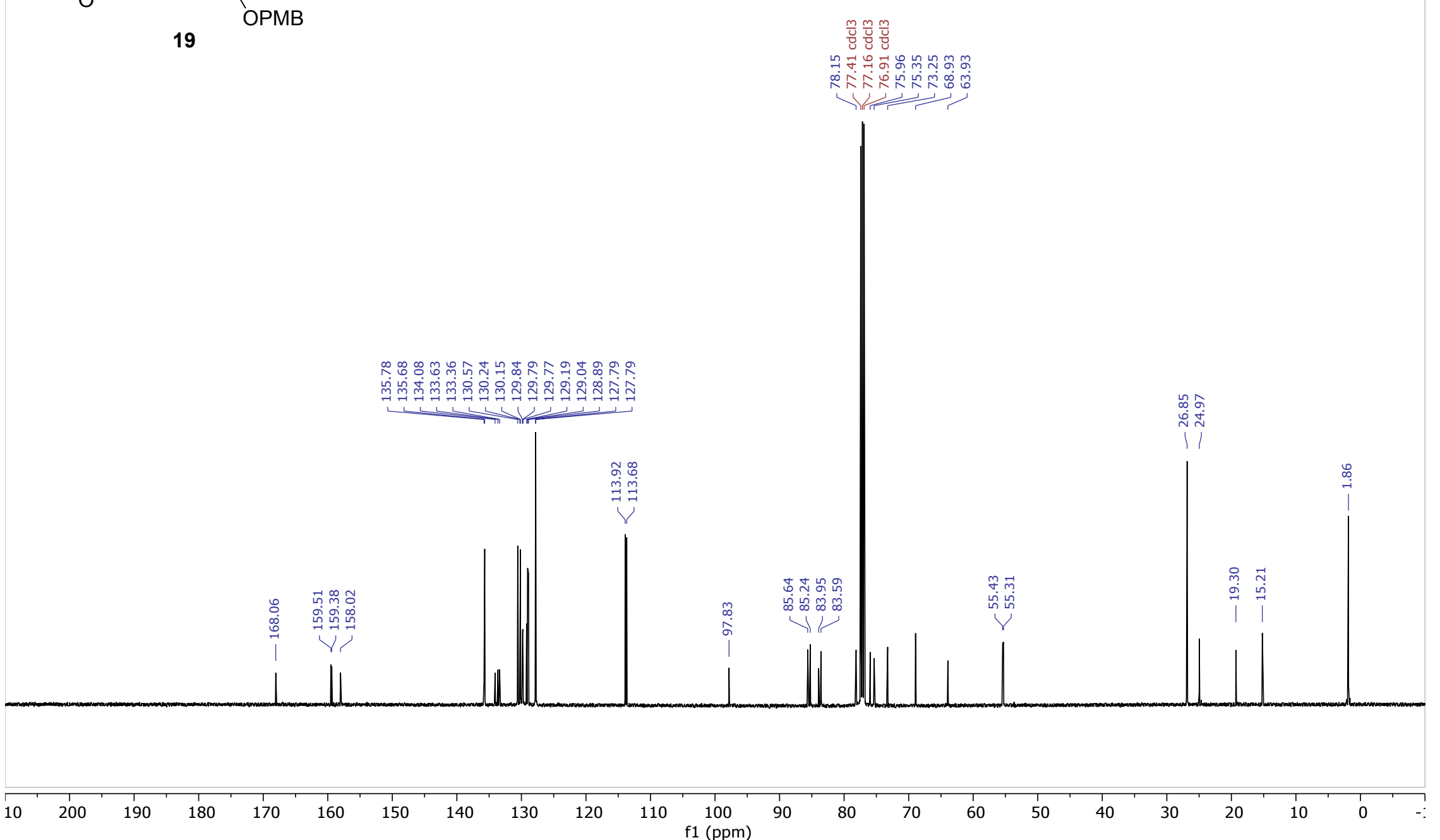
${ }^{1} \mathrm{H}-\mathrm{NMR}\left(500 \mathrm{MHz}, \mathrm{CDCl}_{3}\right.$ )

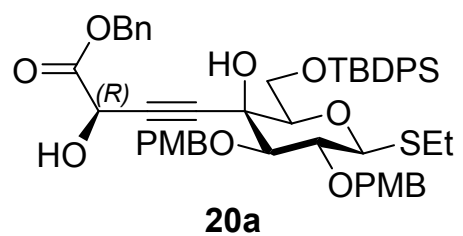

20a

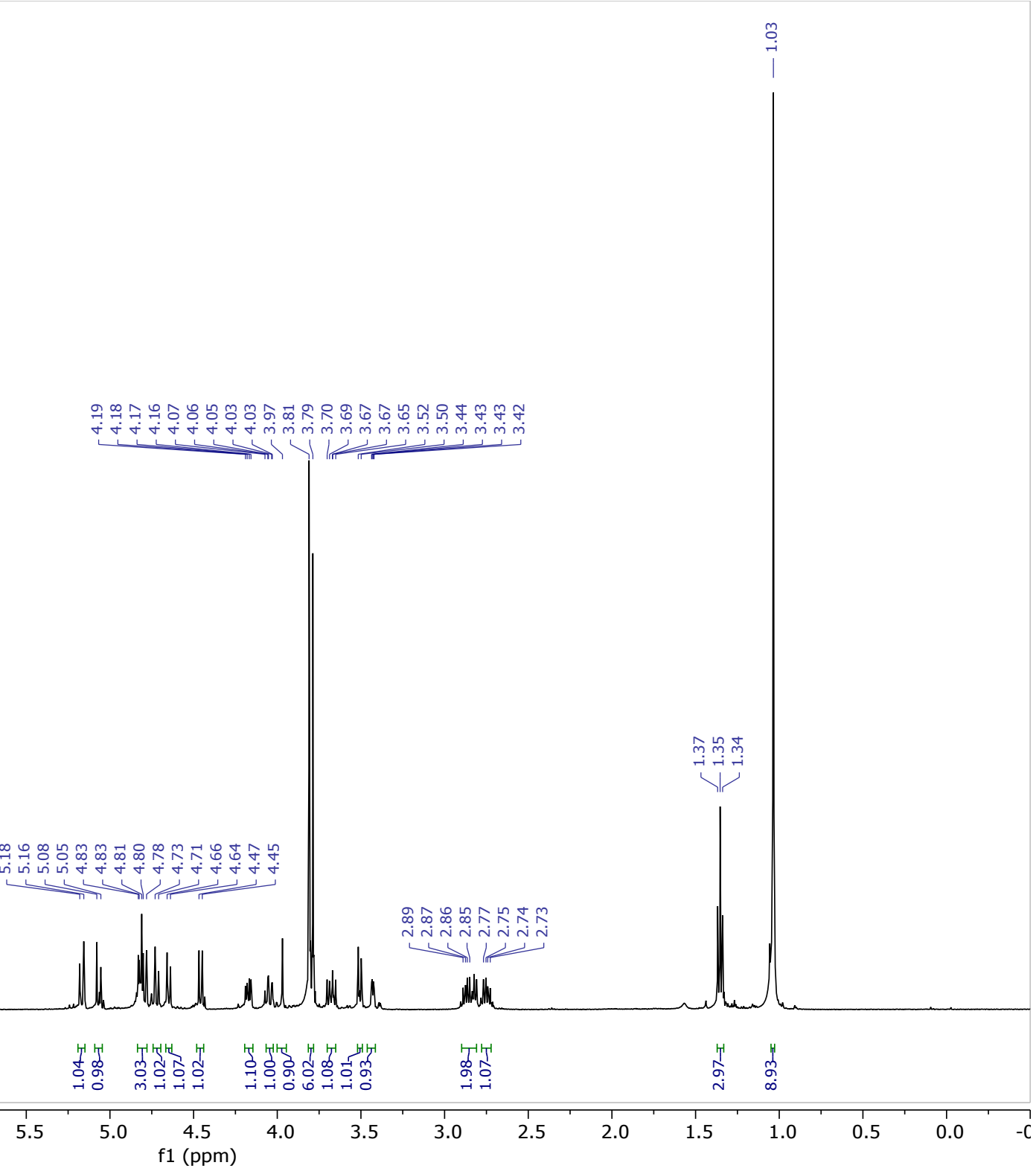


${ }^{13} \mathrm{C}\left\{{ }^{1} \mathrm{H}\right\}-\mathrm{NMR}\left(126 \mathrm{MHz}, \mathrm{CDCl}_{3}\right.$ )

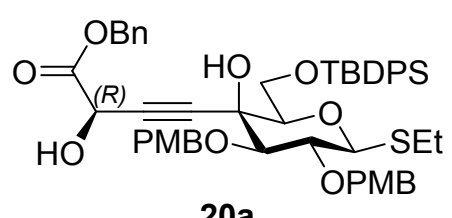

$20 a$
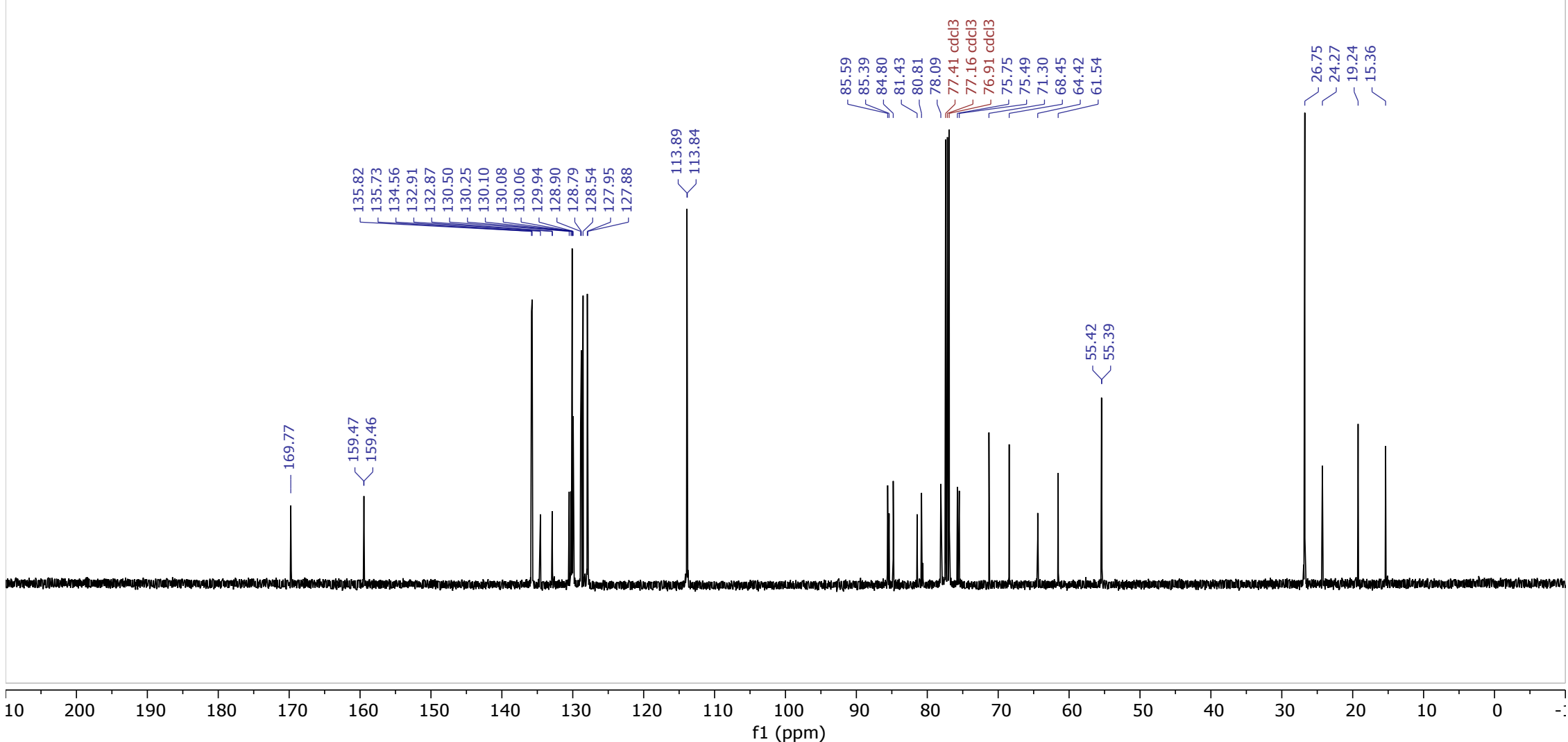
${ }^{1} \mathrm{H}-\mathrm{NMR}\left(500 \mathrm{MHz}, \mathrm{CDCl}_{3}\right)$

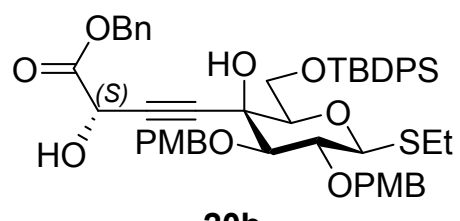

$20 \mathrm{~b}$ OPMB

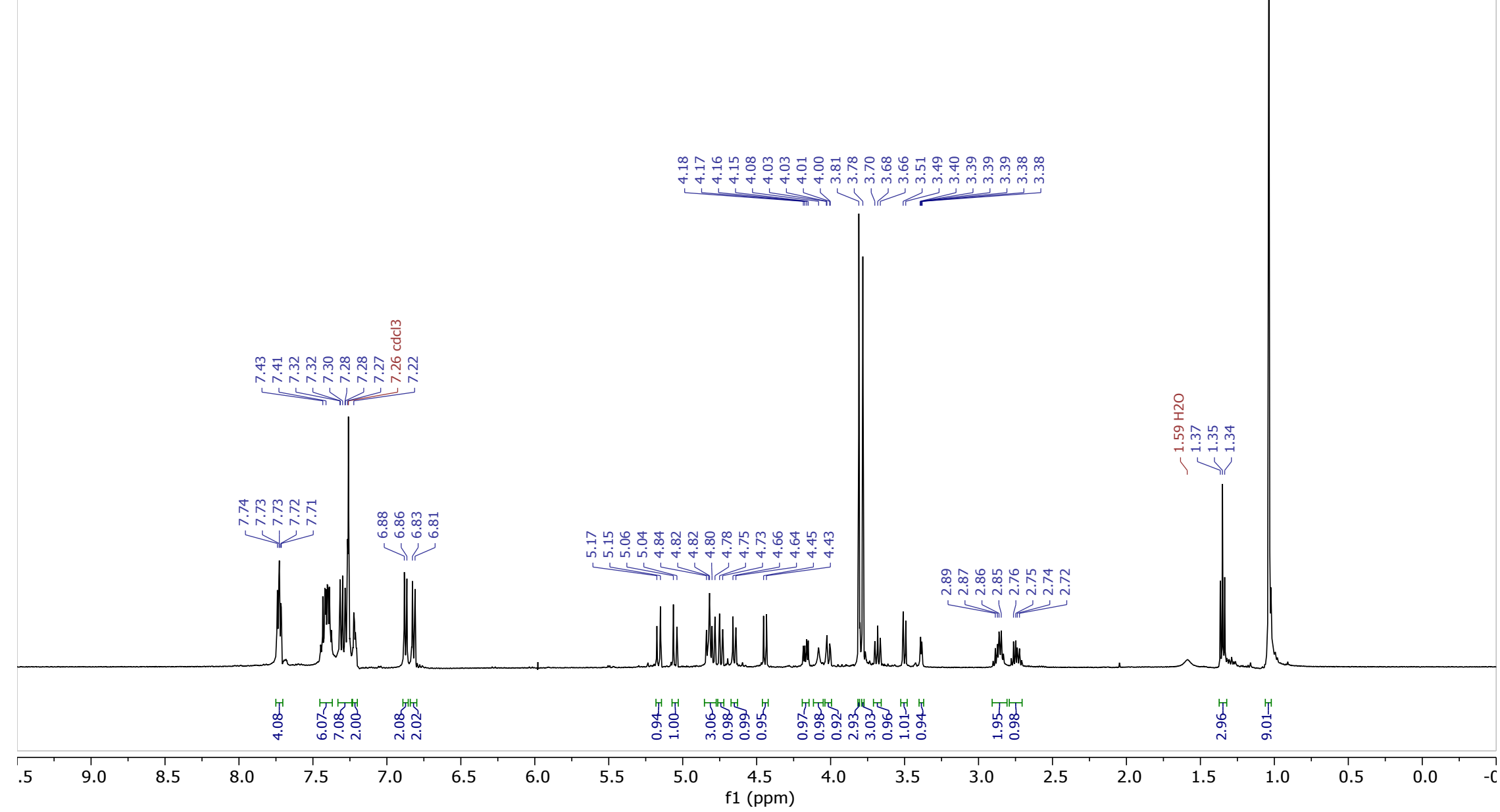



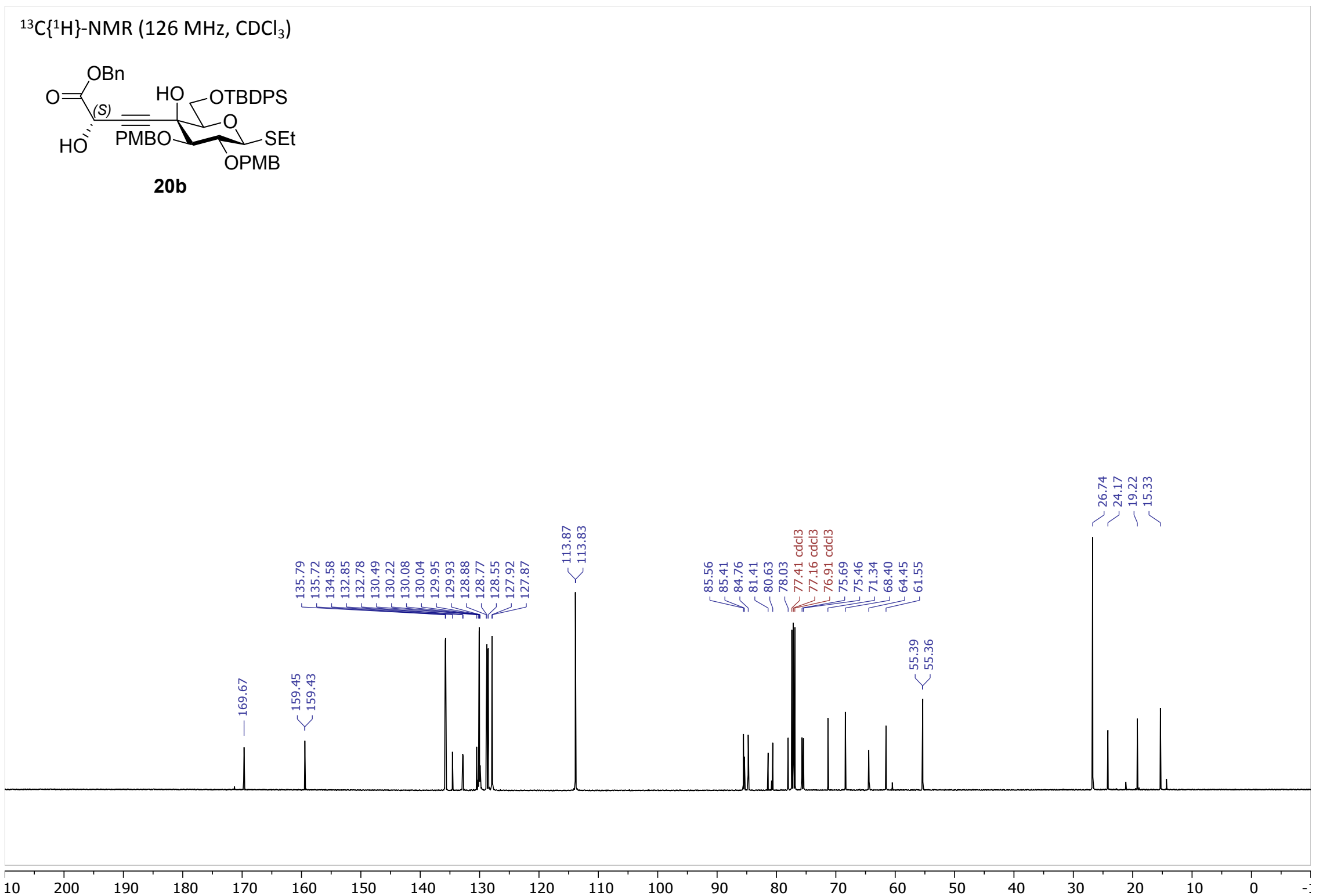
${ }^{1} \mathrm{H}-\mathrm{NMR}\left(500 \mathrm{MHz}, \mathrm{CDCl}_{3}\right)$

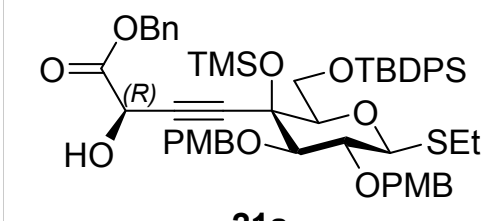

21a

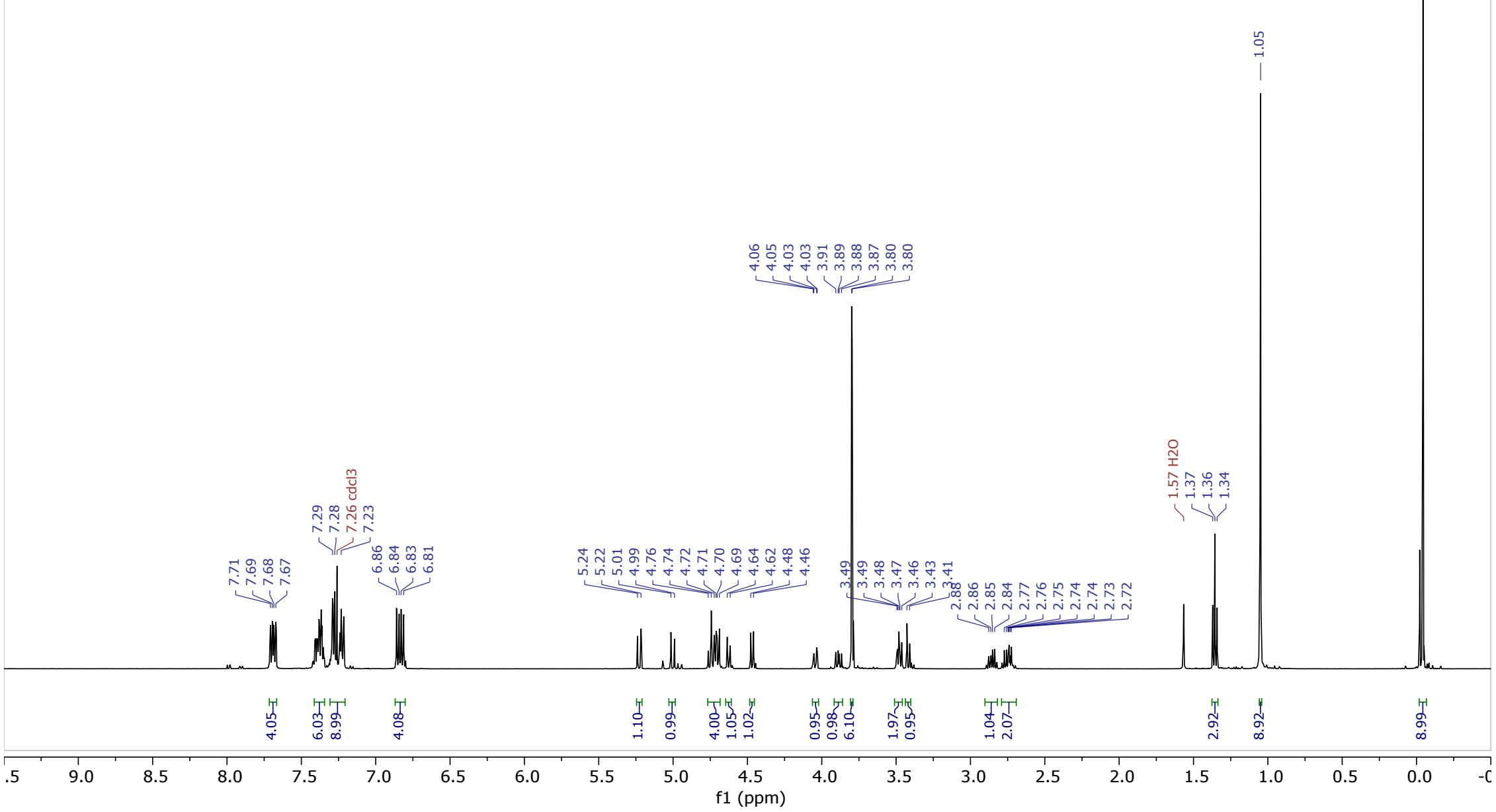


${ }^{13} \mathrm{C}\left\{{ }^{1} \mathrm{H}\right\}-\mathrm{NMR}\left(126 \mathrm{MHz}, \mathrm{CDCl}_{3}\right)$

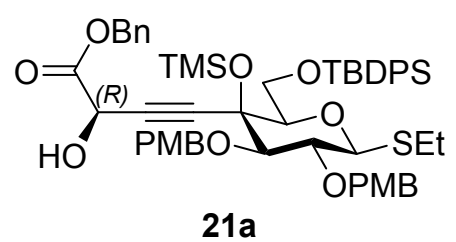

21a

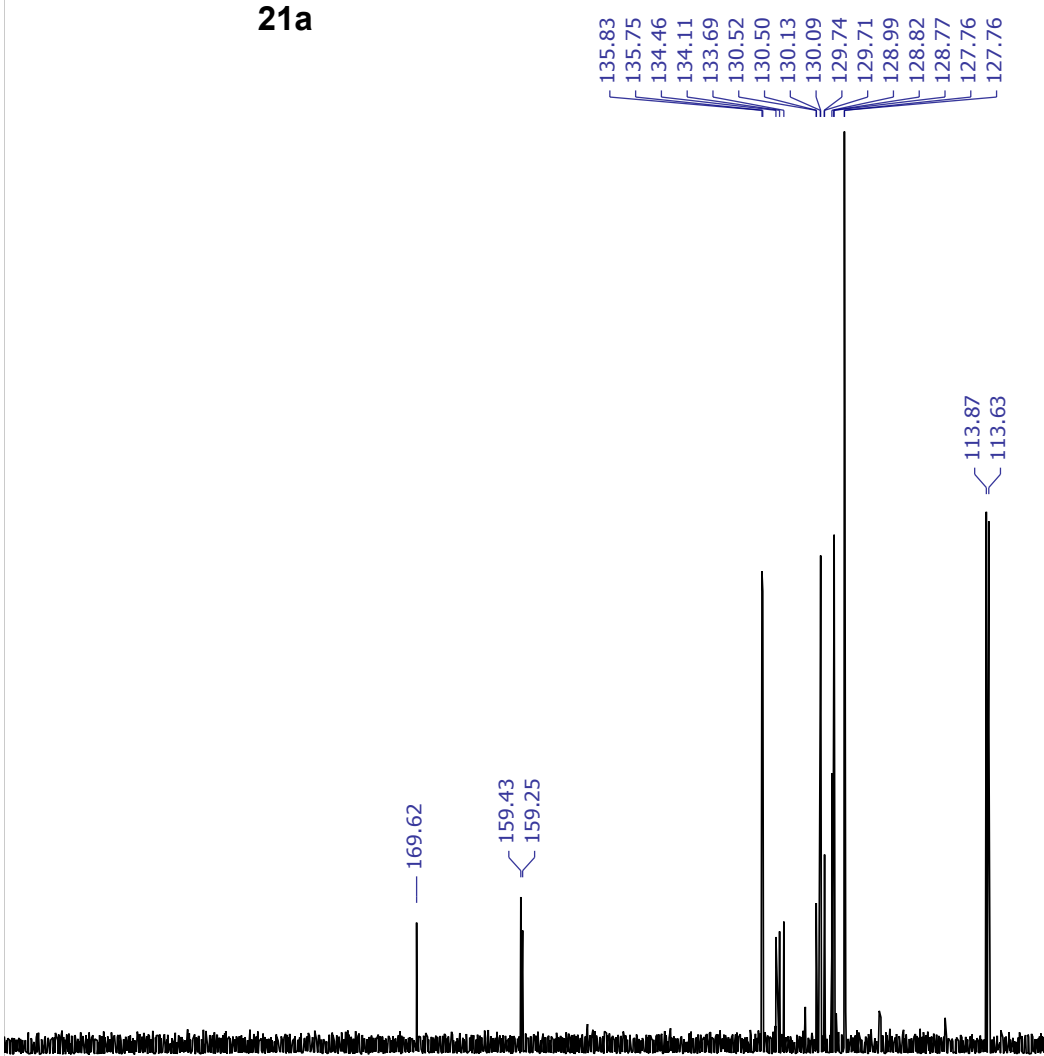

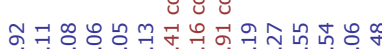

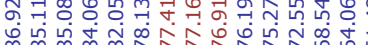

पो।

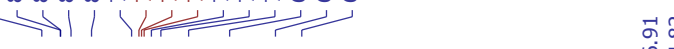

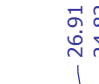


${ }^{1} \mathrm{H}-\mathrm{NMR}\left(500 \mathrm{MHz}, \mathrm{CDCl}_{3}\right.$ )

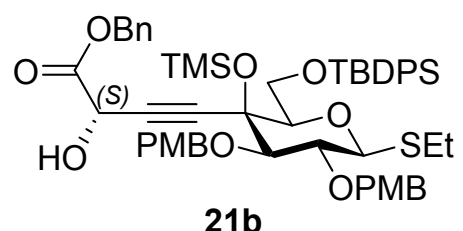

21b

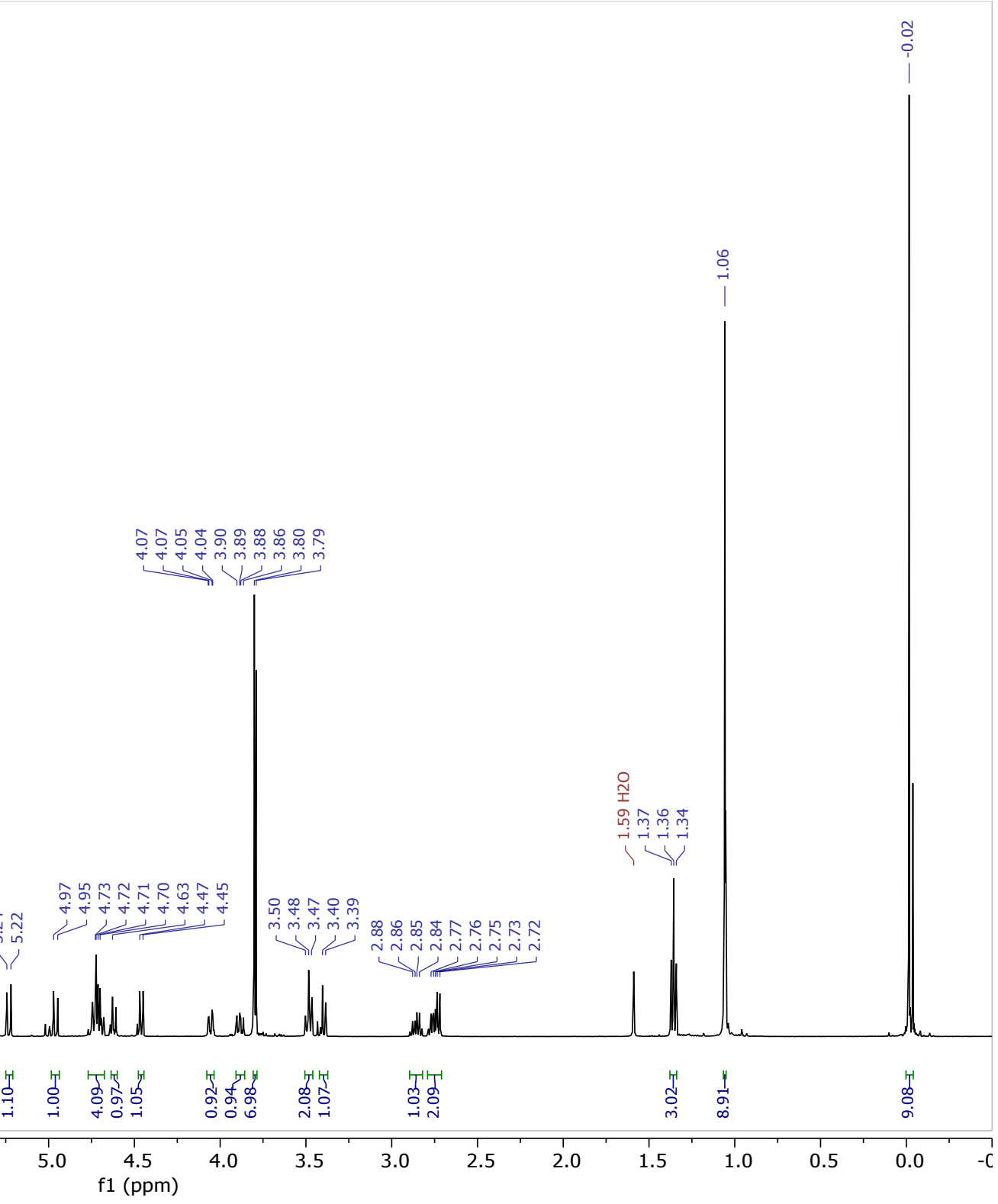


${ }^{13} \mathrm{C}\left\{{ }^{1} \mathrm{H}\right\}-N M R\left(126 \mathrm{MHz}, \mathrm{CDCl}_{3}\right)$
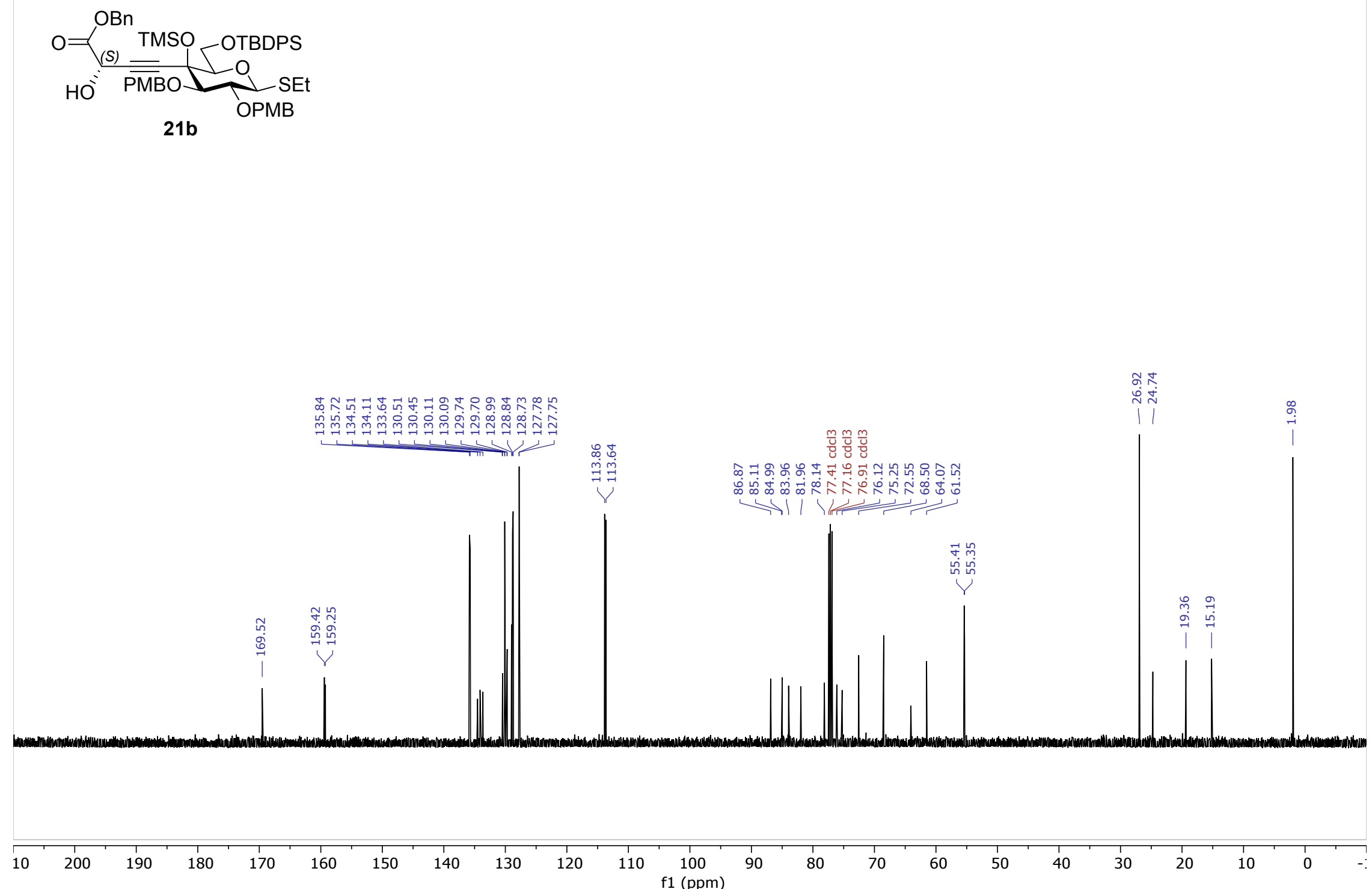
${ }^{1} \mathrm{H}-\mathrm{NMR}\left(500 \mathrm{MHz}, \mathrm{CDCl}_{3}\right)$

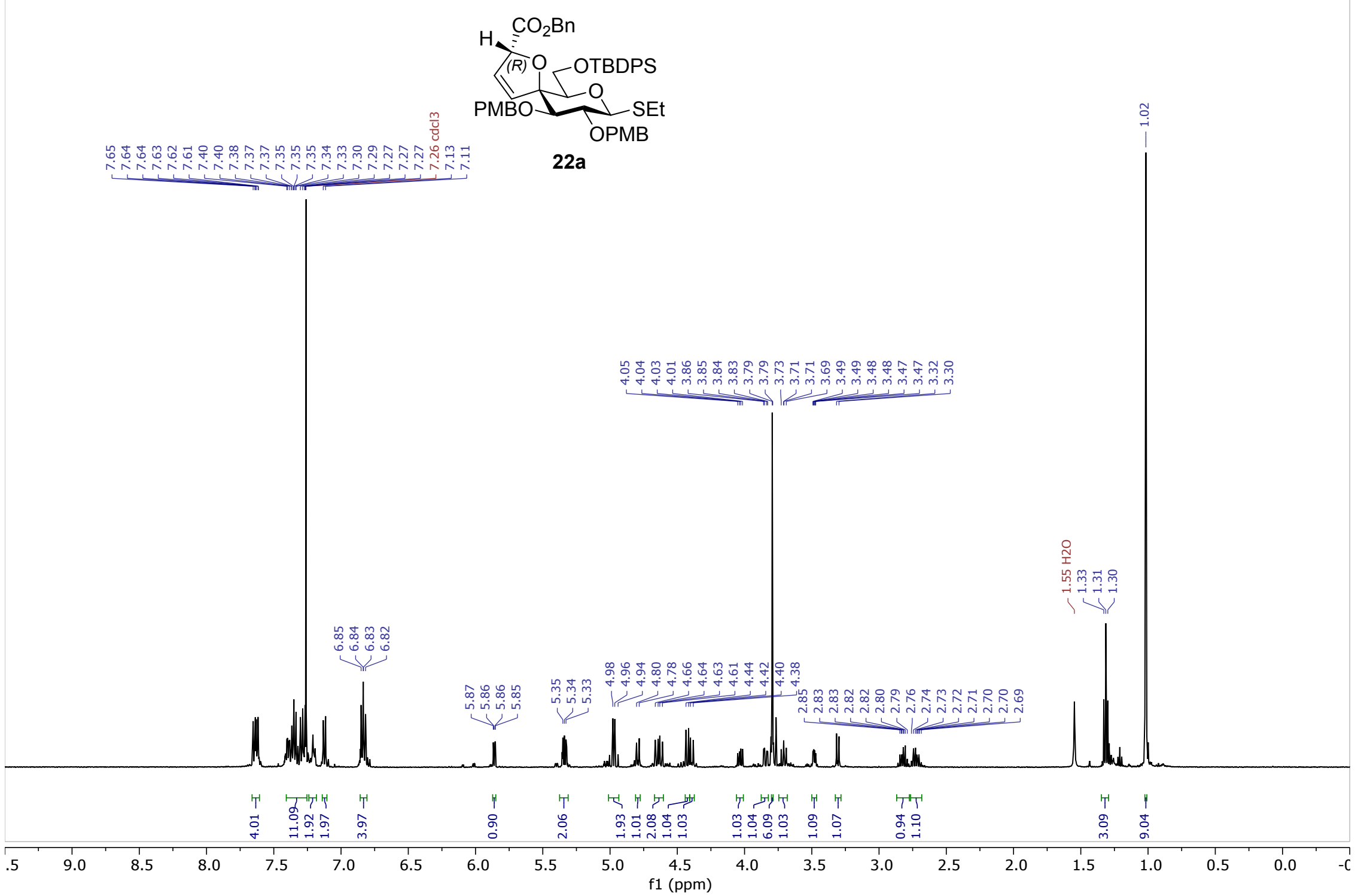


${ }^{13} \mathrm{C}\left\{{ }^{1} \mathrm{H}\right\}-\mathrm{NMR}\left(126 \mathrm{MHz}, \mathrm{CDCl}_{3}\right)$

$\mathrm{H} \cong \mathrm{CO}_{2} \mathrm{Bn}$

(R) O OTBDPS

SEt

OPMB

22a

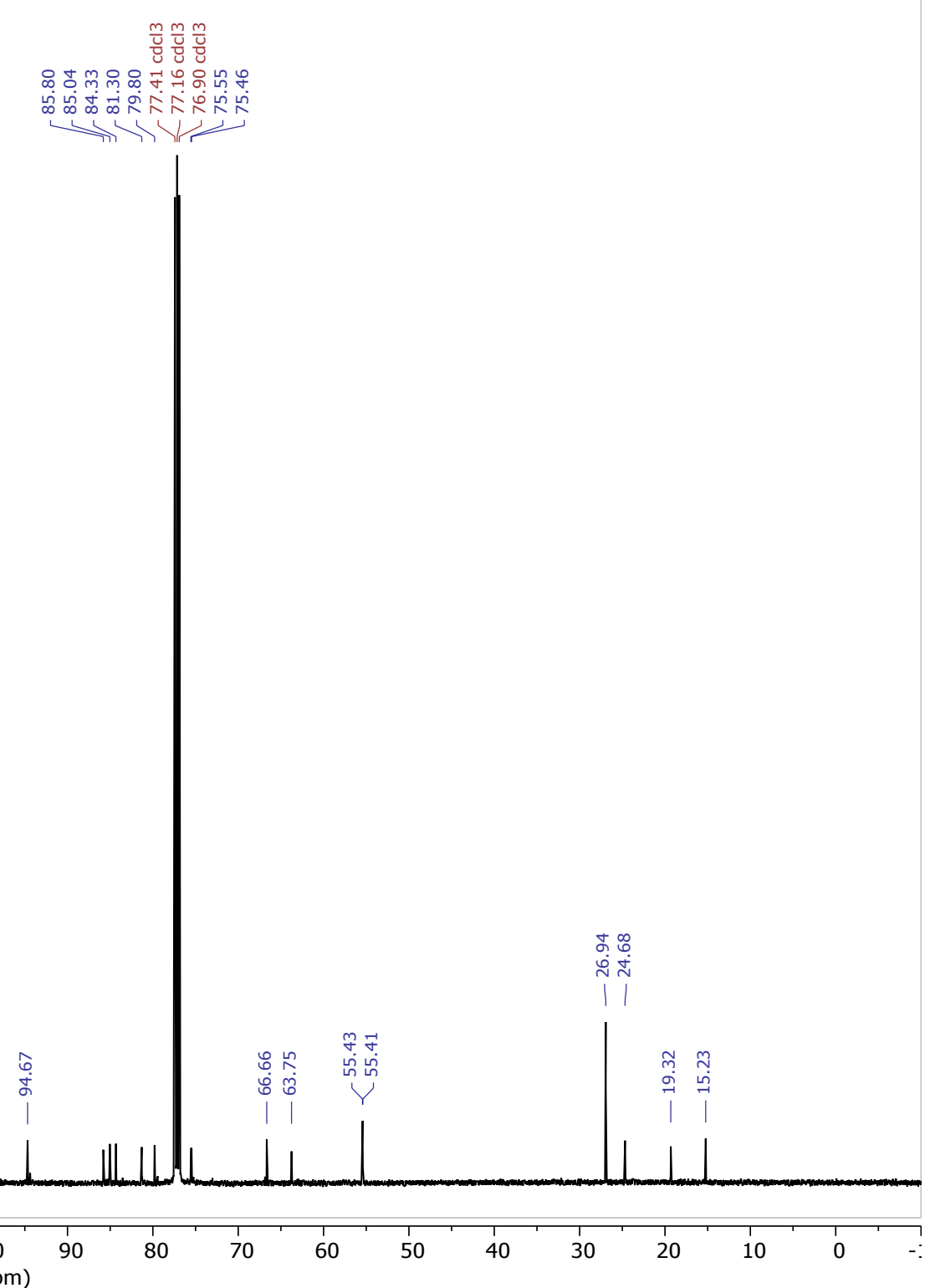


${ }^{1} \mathrm{H}-\mathrm{NMR}\left(500 \mathrm{MHz}, \mathrm{CDCl}_{3}\right)$

$\mathrm{H}, \mathrm{CO}_{2} \mathrm{Bn}$

(S) O OOTBDPS

PMBO

OPMB

22b

$\frac{\bar{m}}{0}$

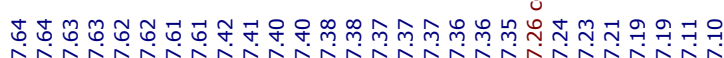

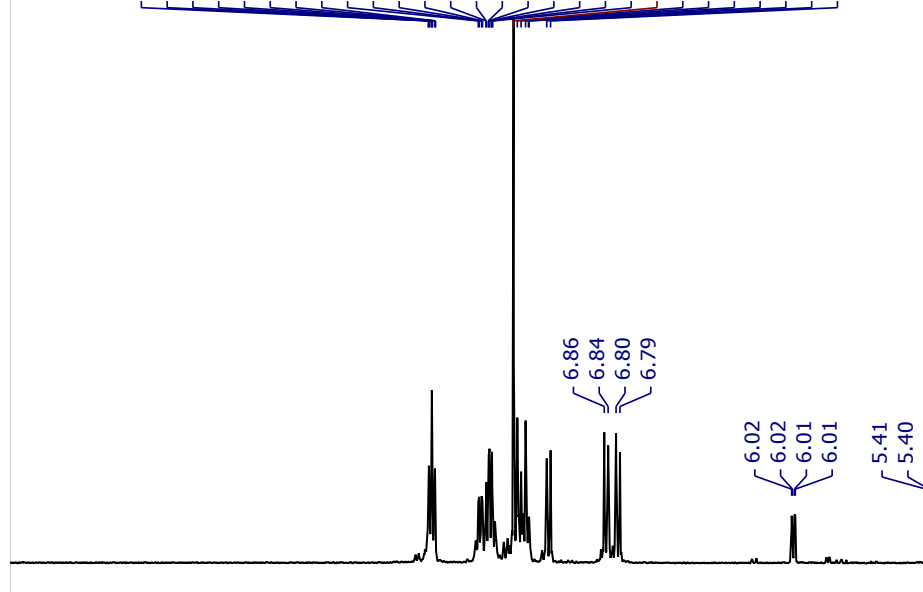

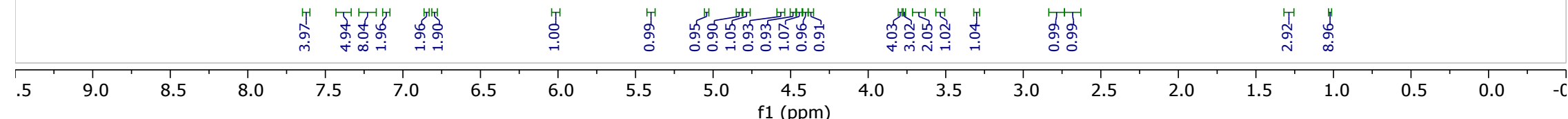


${ }^{13} \mathrm{C}\left\{{ }^{1} \mathrm{H}\right\}-\mathrm{NMR}\left(126 \mathrm{MHz}, \mathrm{CDCl}_{3}\right)$

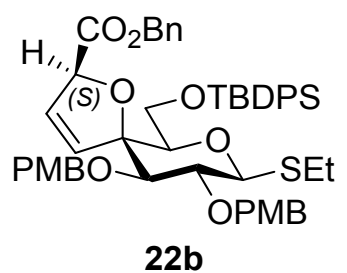

22b

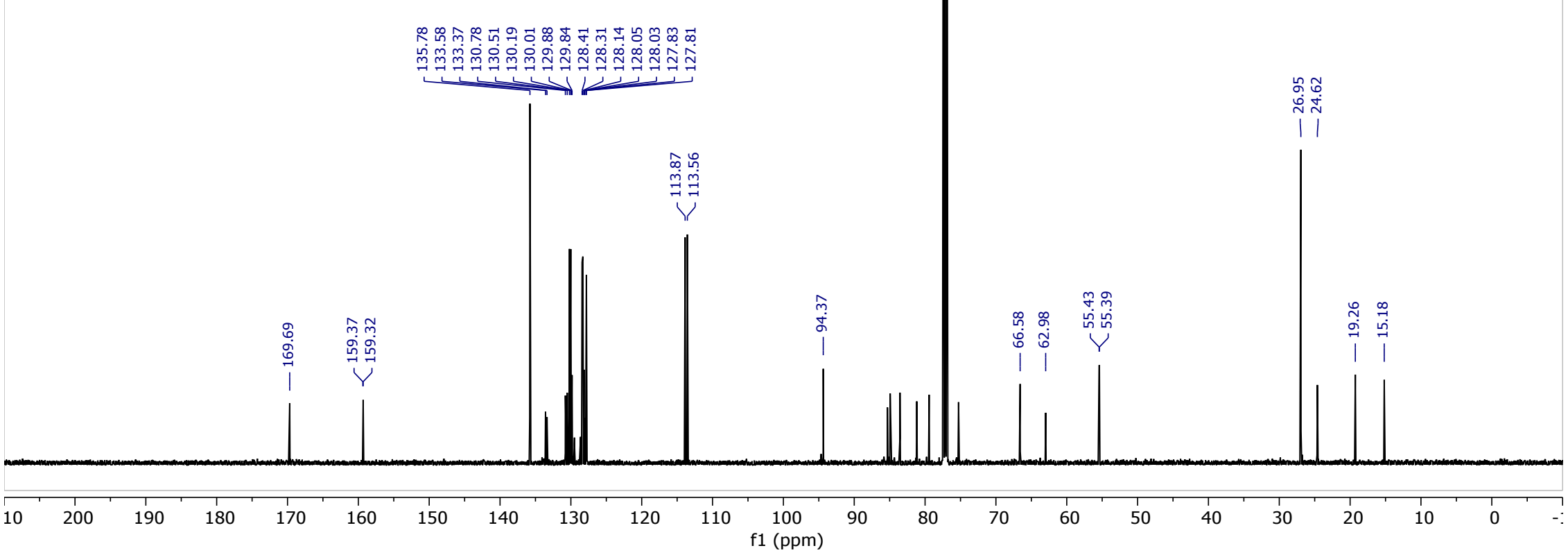




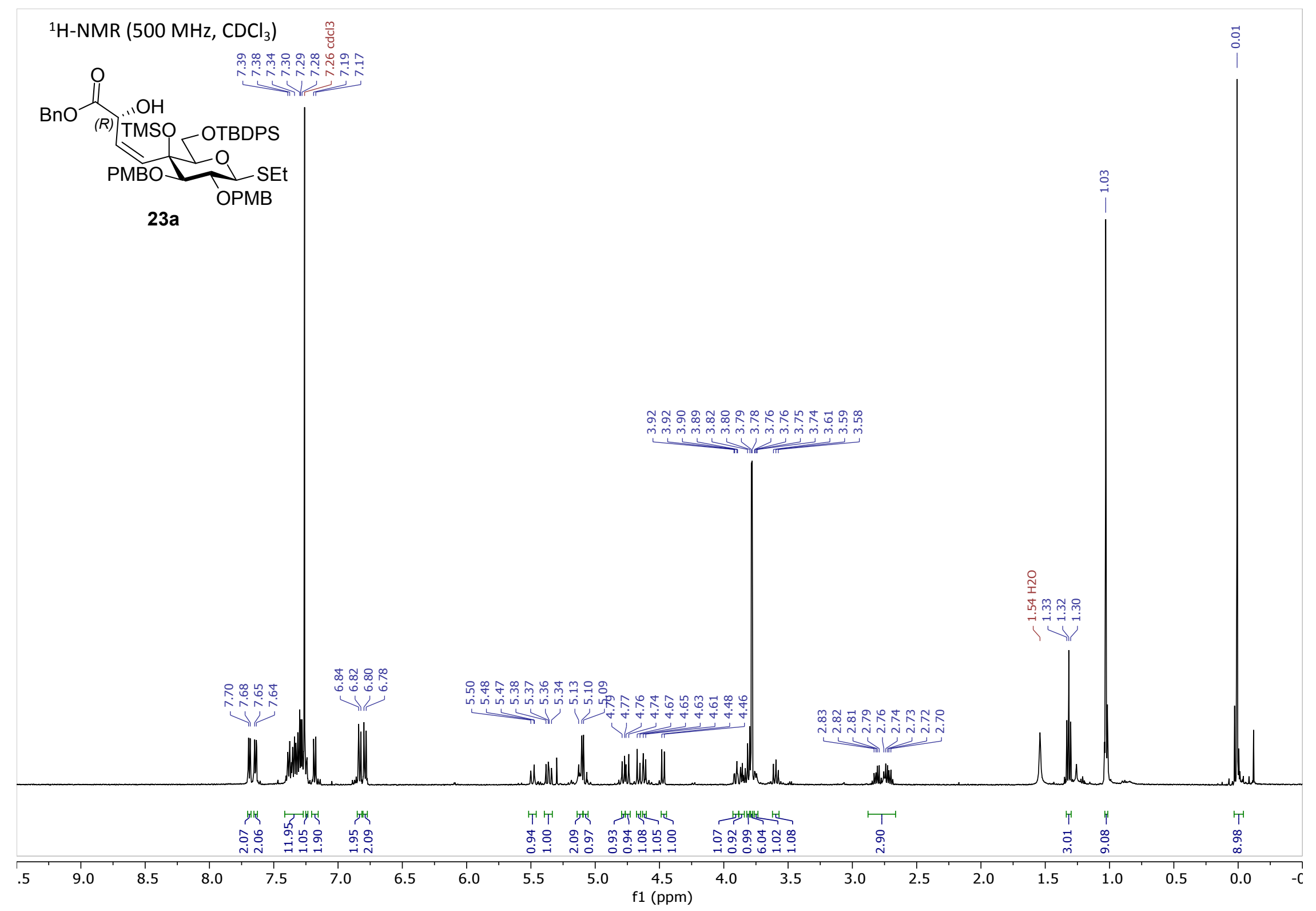




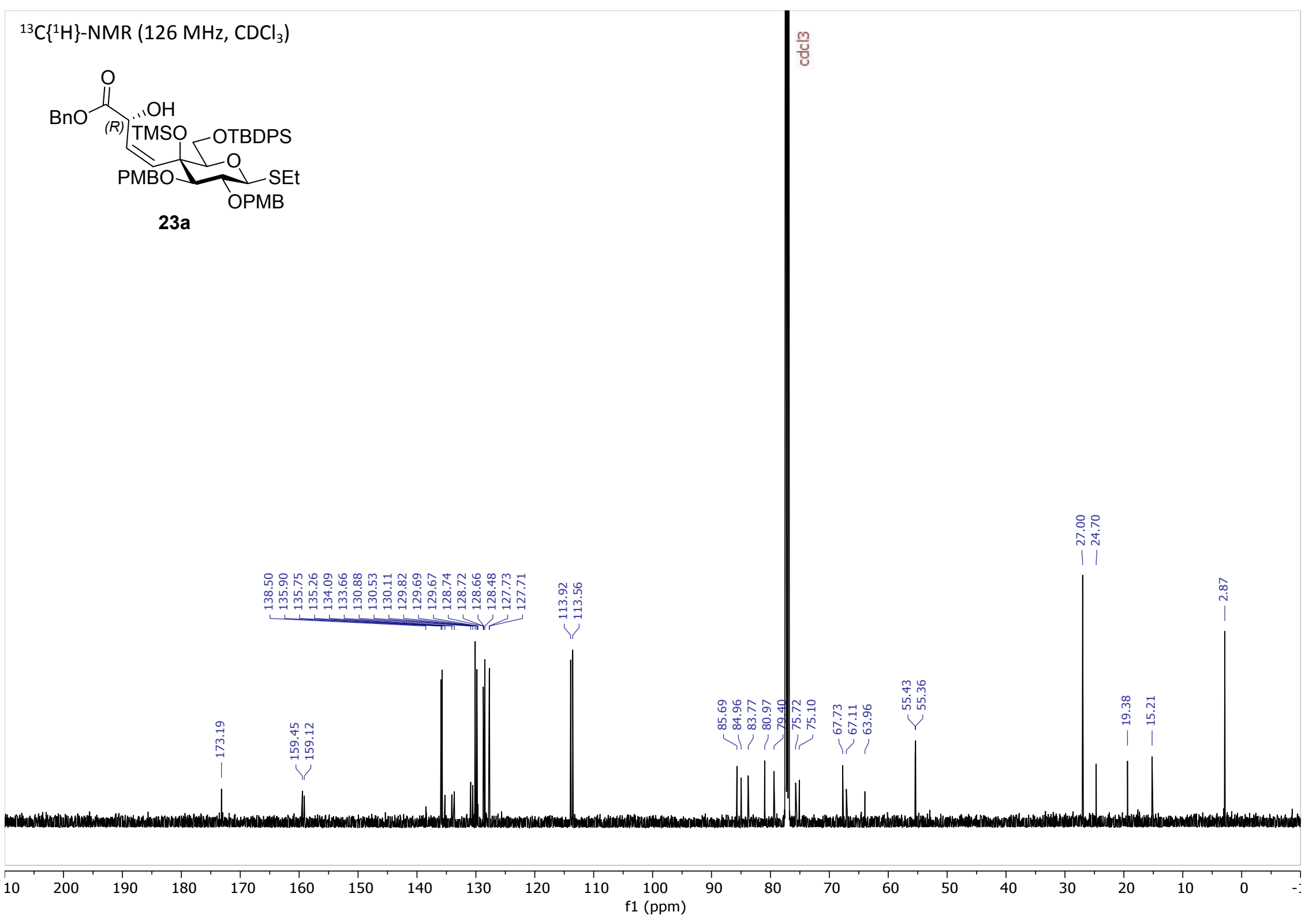


${ }^{1} \mathrm{H}-\mathrm{NMR}\left(500 \mathrm{MHz}, \mathrm{CDCl}_{3}\right)$

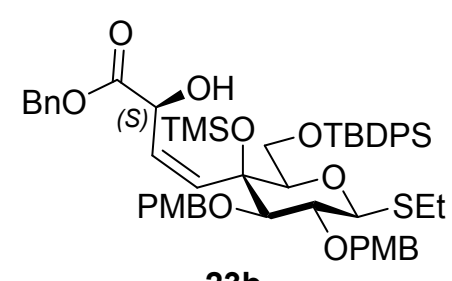

23b

$\frac{m}{8}$

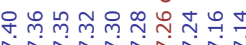

신.

mill

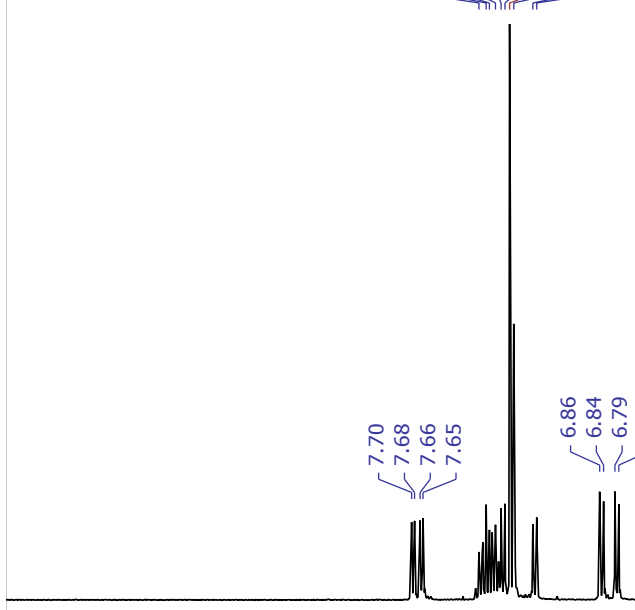

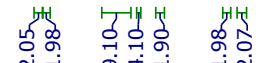

岗菂品

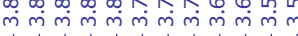

(1)

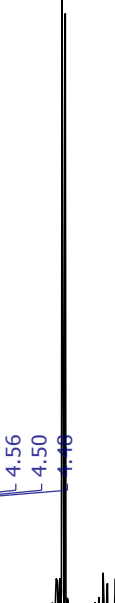

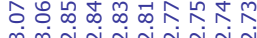
Luandiluldulal| Wh the the

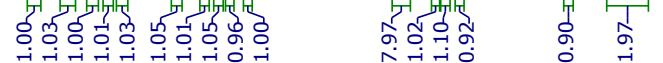
$5.5 \quad 5.0$ 
${ }^{13} \mathrm{C}\left\{{ }^{1} \mathrm{H}\right\}-\mathrm{NMR}\left(126 \mathrm{MHz}, \mathrm{CDCl}_{3}\right)$

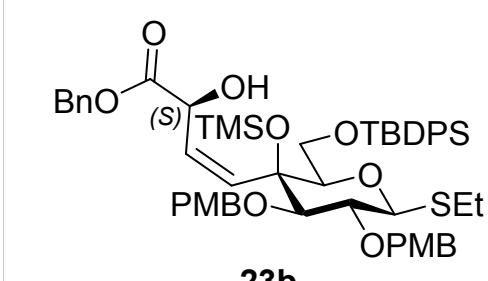

23b

PMB
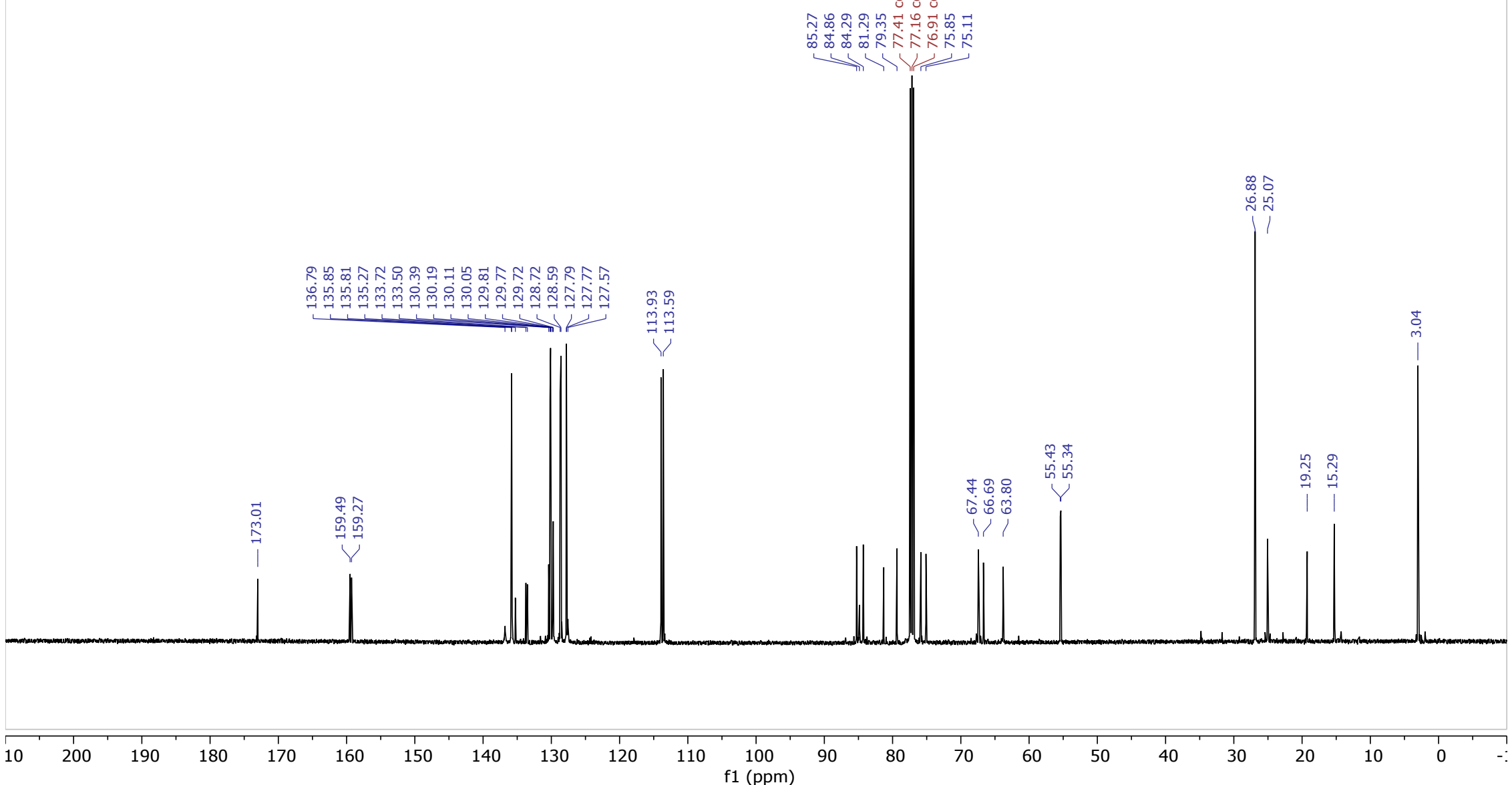
${ }^{1} \mathrm{H}-\mathrm{NMR}\left(500 \mathrm{MHz}, \mathrm{CDCl}_{3}\right.$ )

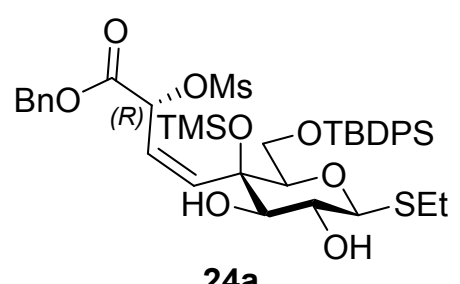

$24 a$

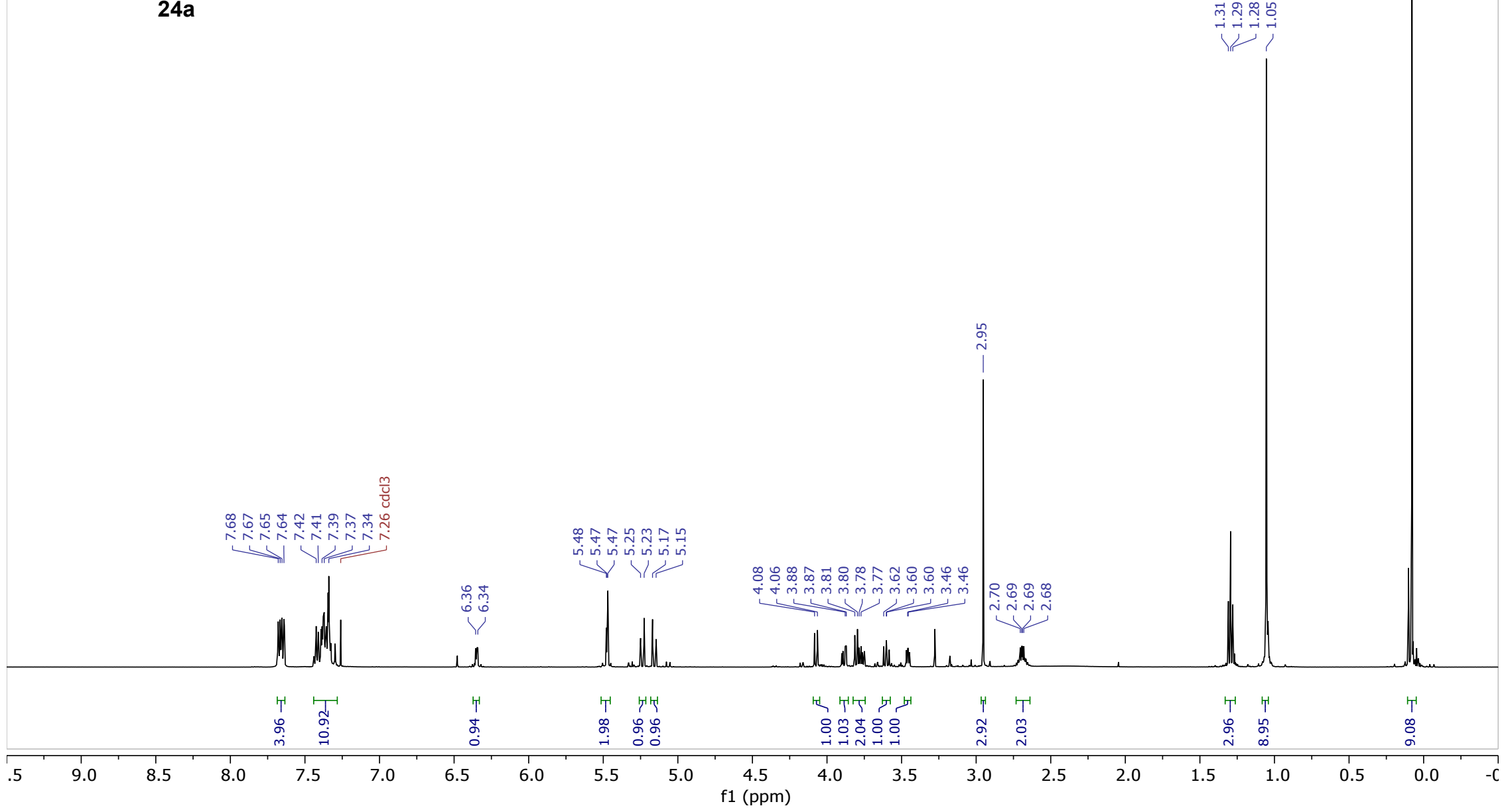


${ }^{13} \mathrm{C}\left\{{ }^{1} \mathrm{H}\right\}-N M R\left(126 \mathrm{MHz}, \mathrm{CDCl}_{3}\right)$

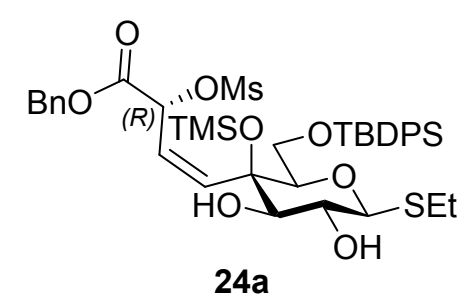

24a
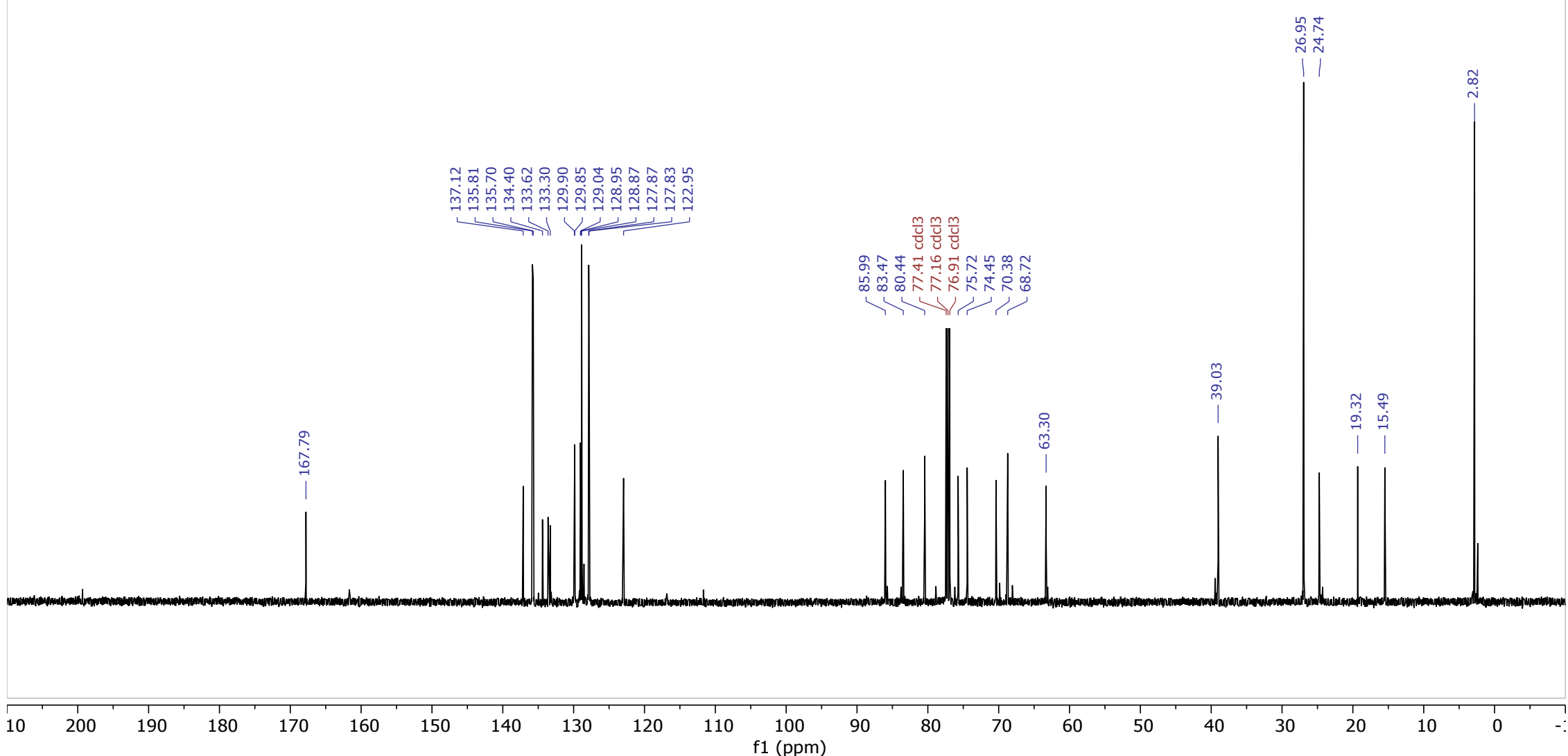
${ }^{1} \mathrm{H}-\mathrm{NMR}\left(500 \mathrm{MHz}, \mathrm{CDCl}_{3}\right)$

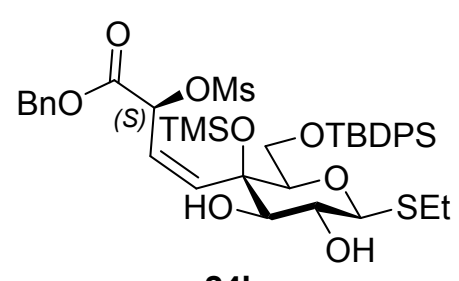

24b

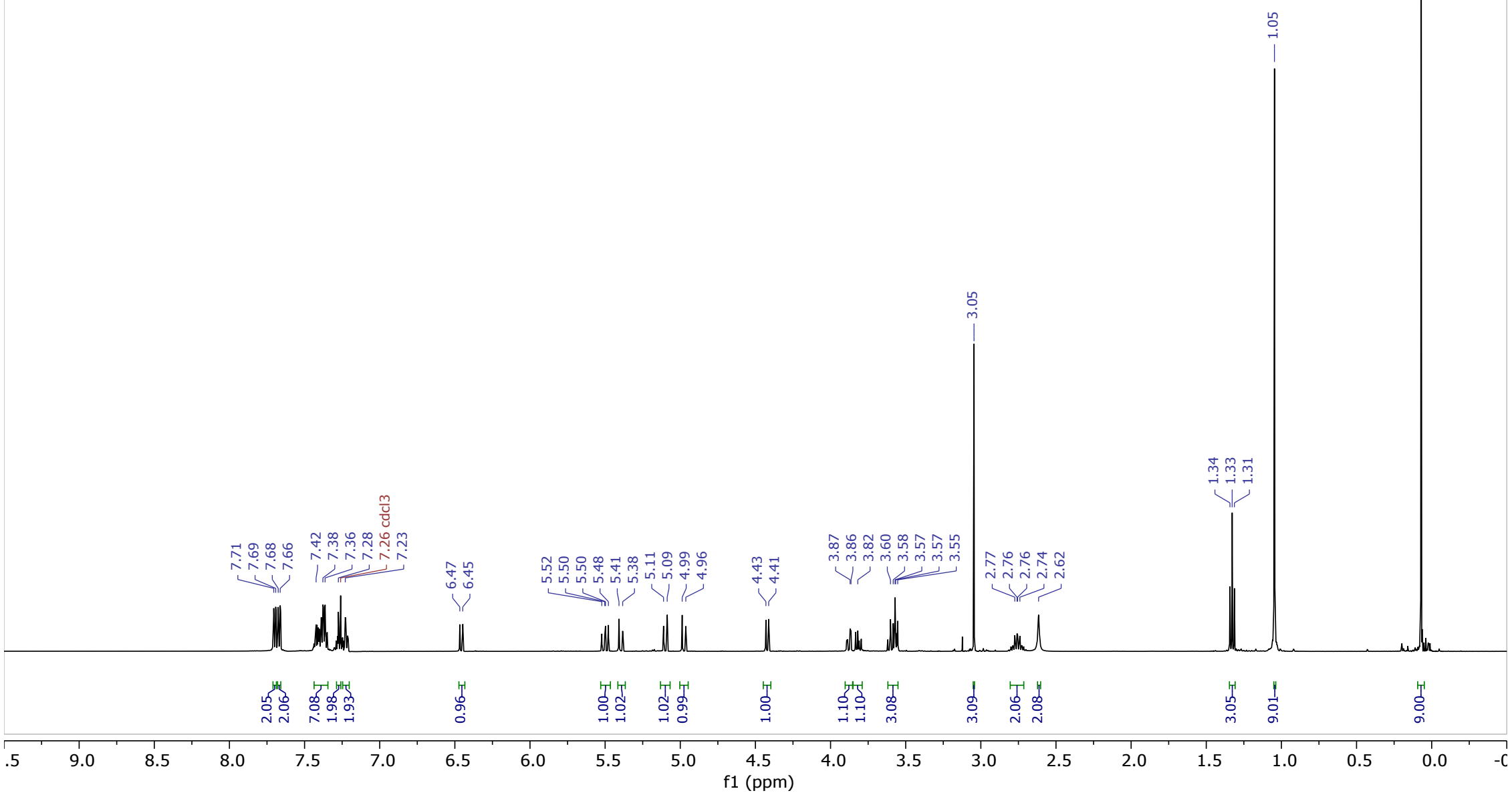


${ }^{13} \mathrm{C}\left\{{ }^{1} \mathrm{H}\right\}-N M R\left(126 \mathrm{MHz}, \mathrm{CDCl}_{3}\right)$

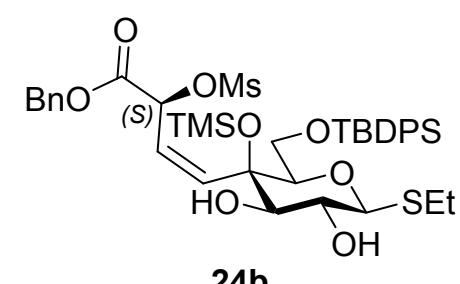

24b

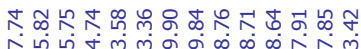

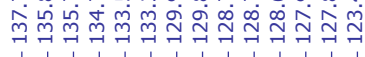

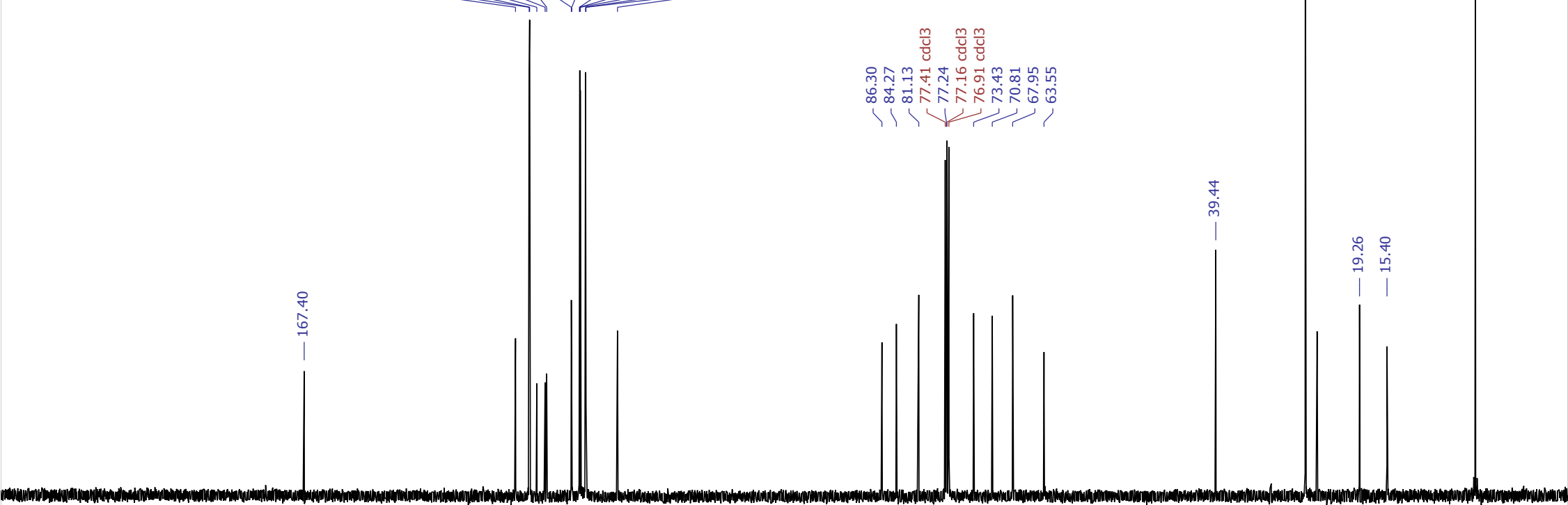

\begin{tabular}{llllllll}
\hline 10 & 200 & 190 & 180 & 170 & 160 & 150 & 140
\end{tabular}

130

120

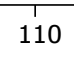

100
f1 $1(\mathrm{ppm})$

$90 \quad 80$

$80 \quad 70$

60

50

40

$30 \quad 20$

10 
${ }^{1} \mathrm{H}-\mathrm{NMR}\left(500 \mathrm{MHz}, \mathrm{CDCl}_{3}\right.$ )

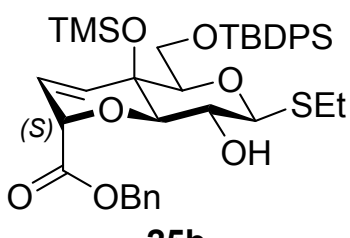

25b

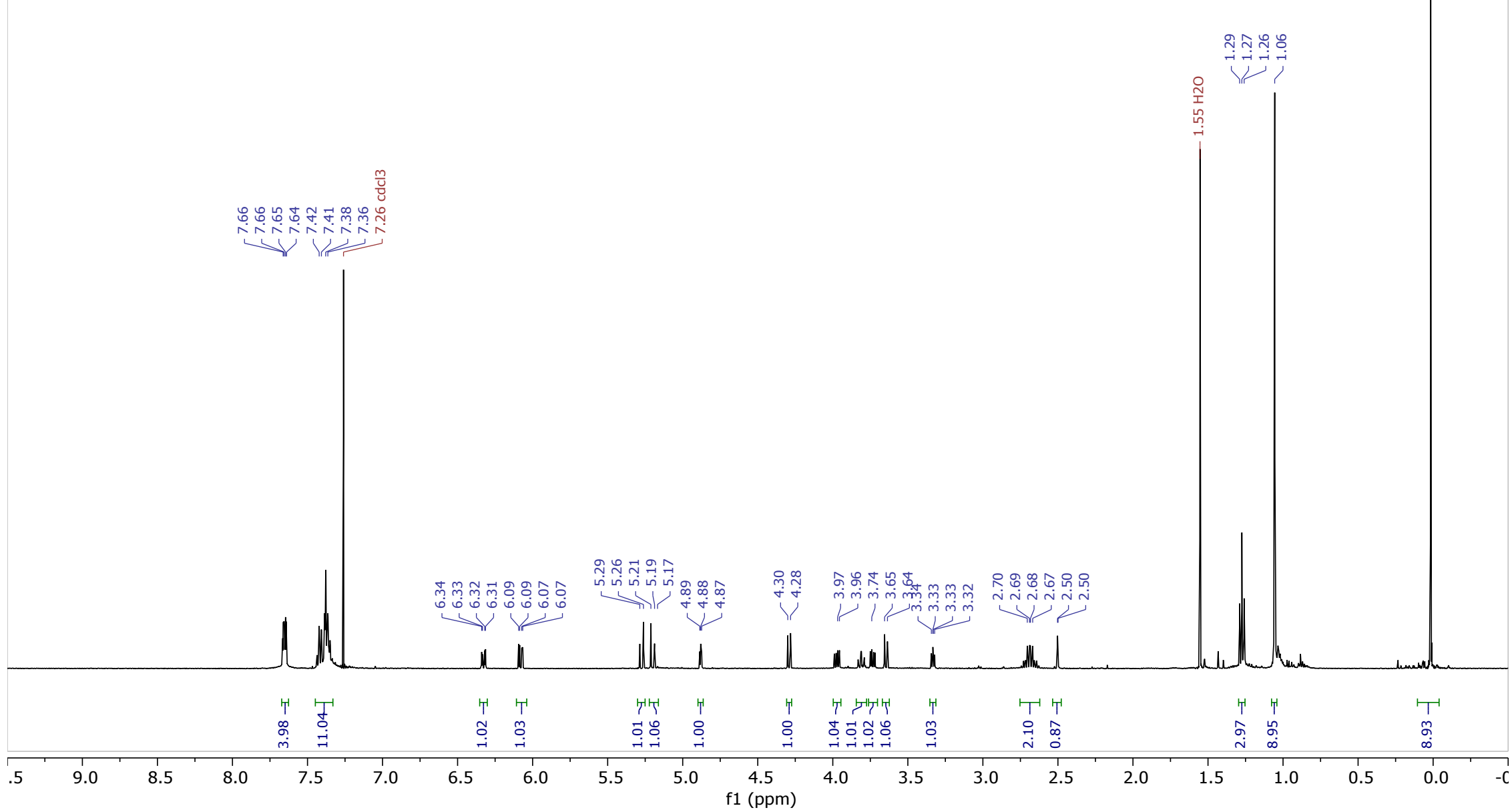


${ }^{13} \mathrm{C}\left\{{ }^{1} \mathrm{H}\right\}-\mathrm{NMR}\left(126 \mathrm{MHz}, \mathrm{CDCl}_{3}\right)$

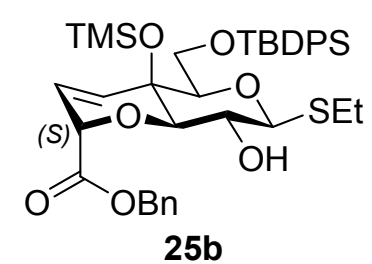

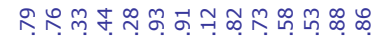

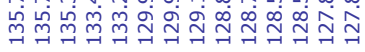
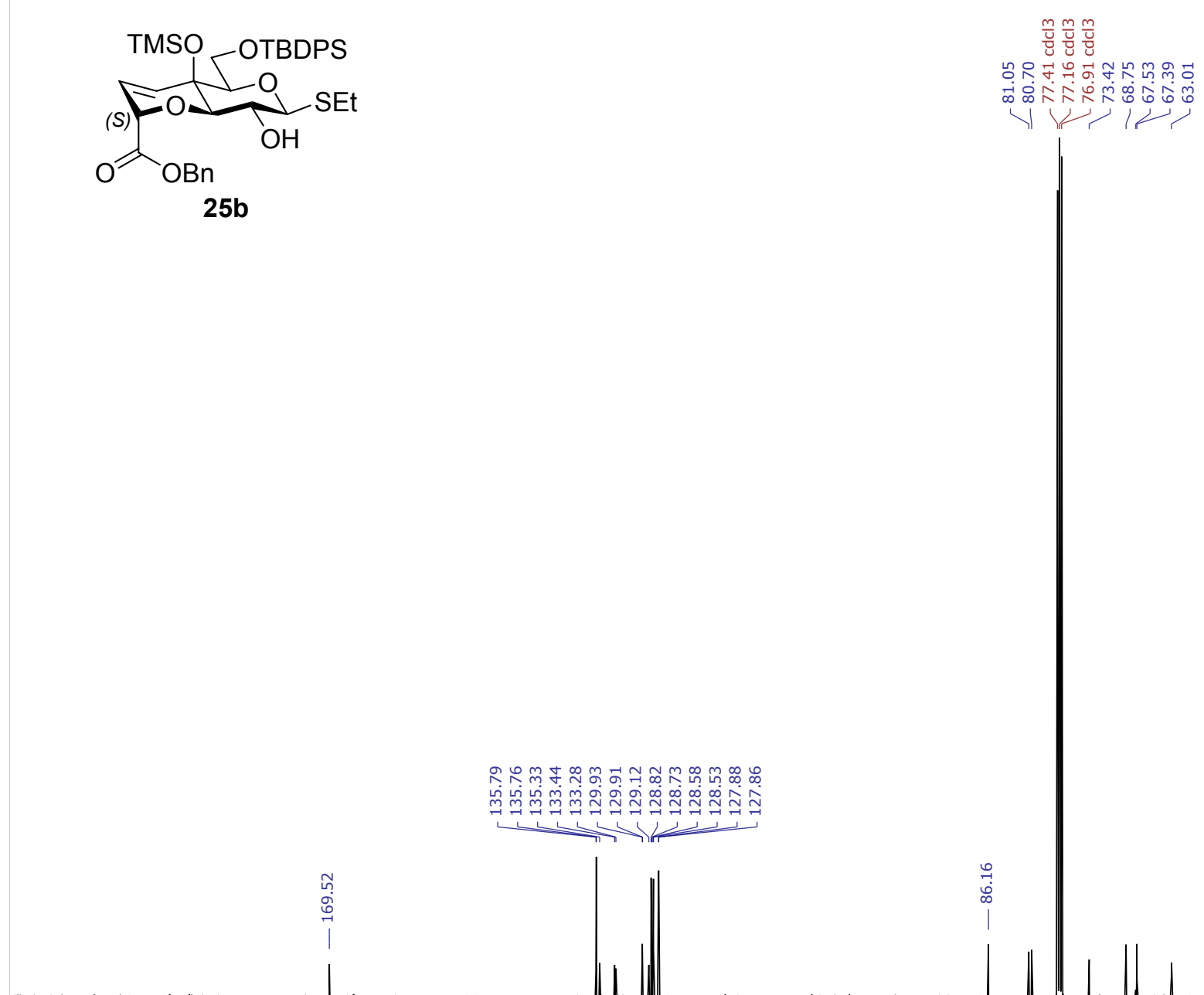

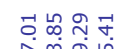

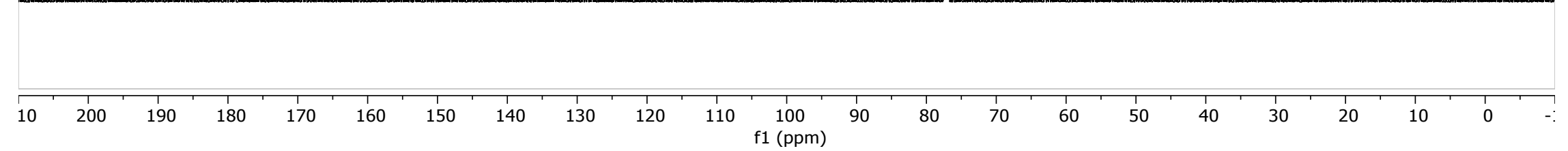




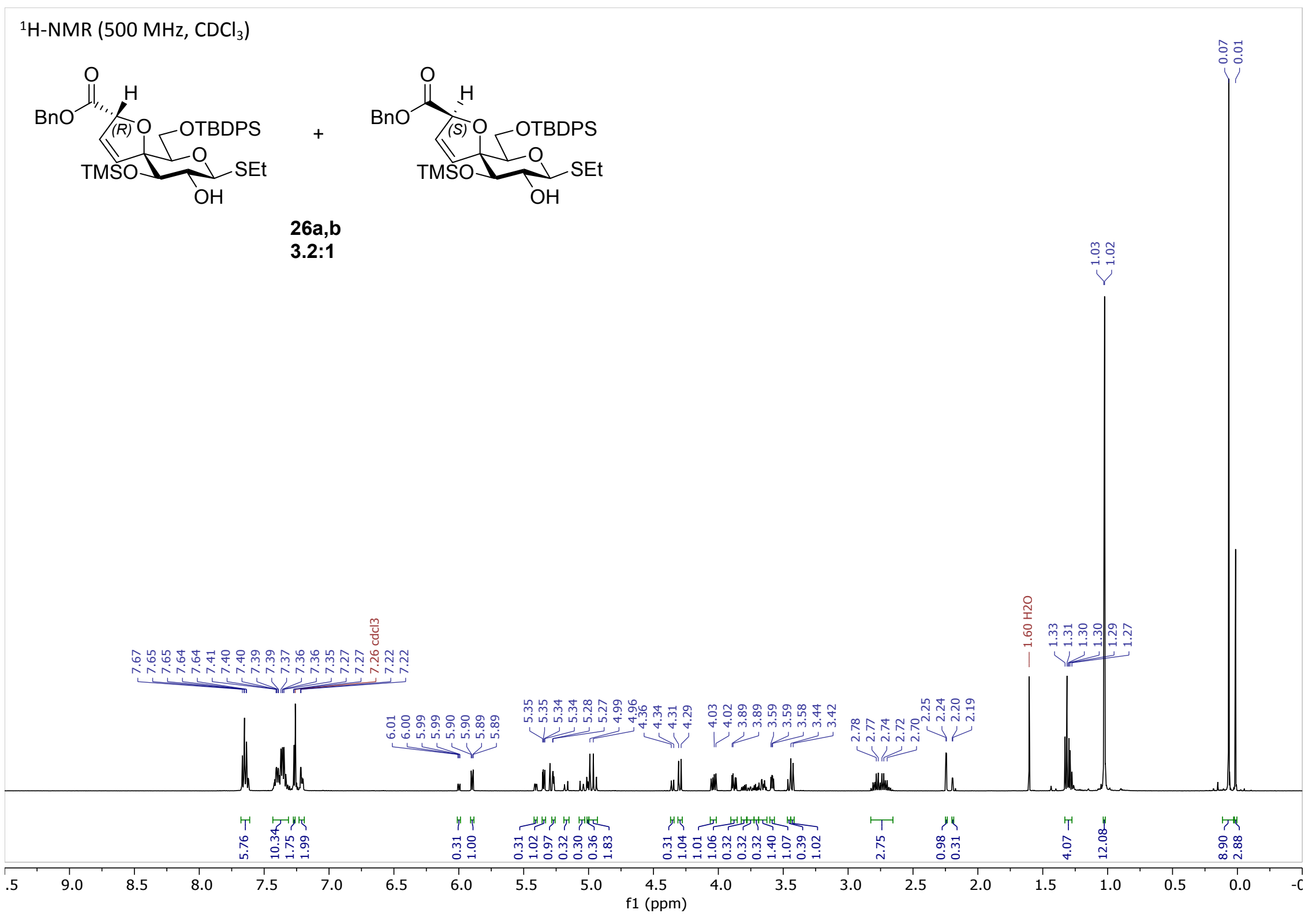


${ }^{13} \mathrm{C}\left\{{ }^{1} \mathrm{H}\right\}-\mathrm{NMR}\left(126 \mathrm{MHz}, \mathrm{CDCl}_{3}\right)$
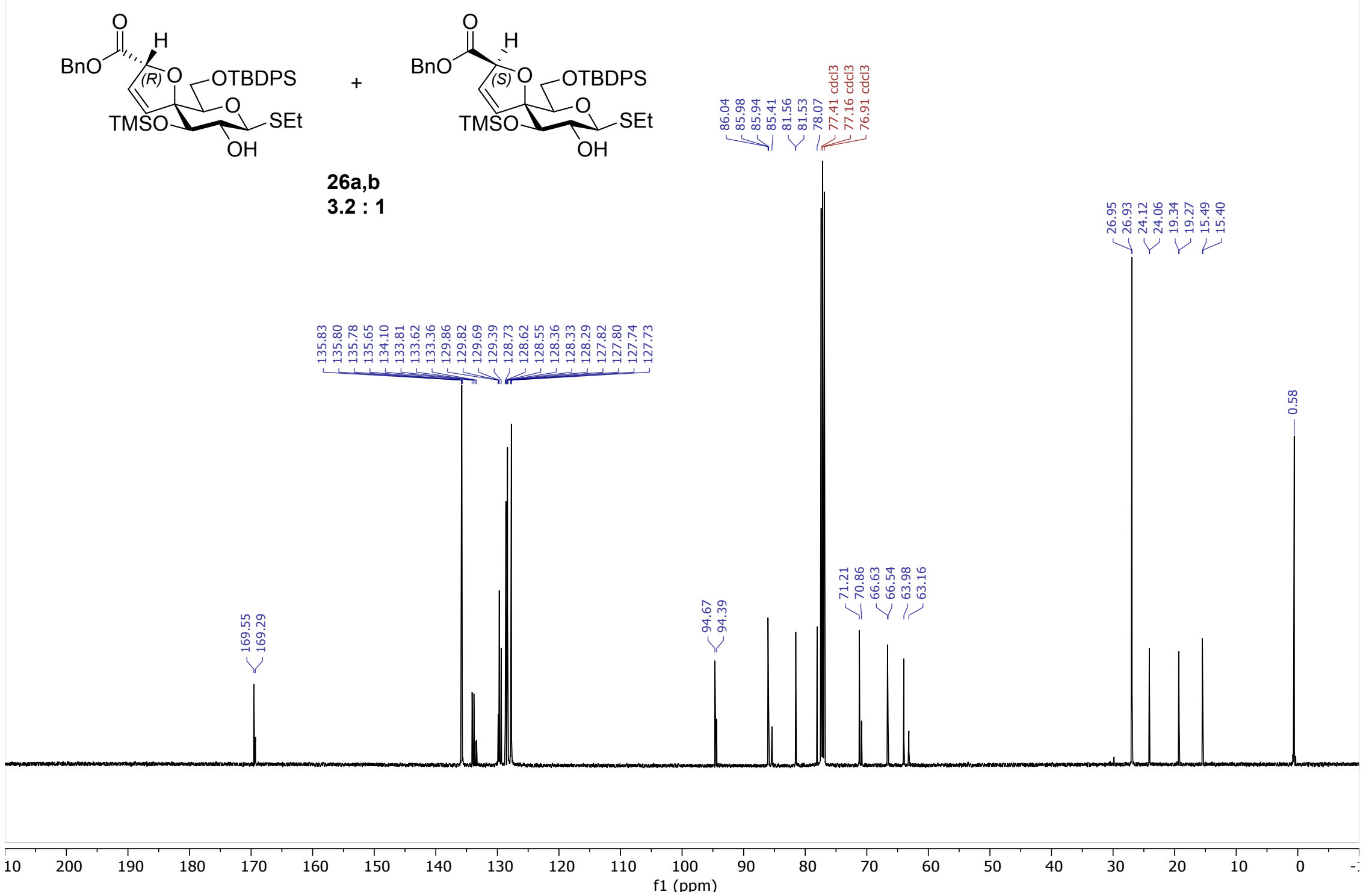
${ }^{1} \mathrm{H}-\mathrm{NMR}\left(500 \mathrm{MHz}, \mathrm{CDCl}_{3}\right)$

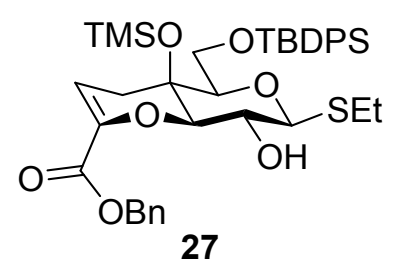

27

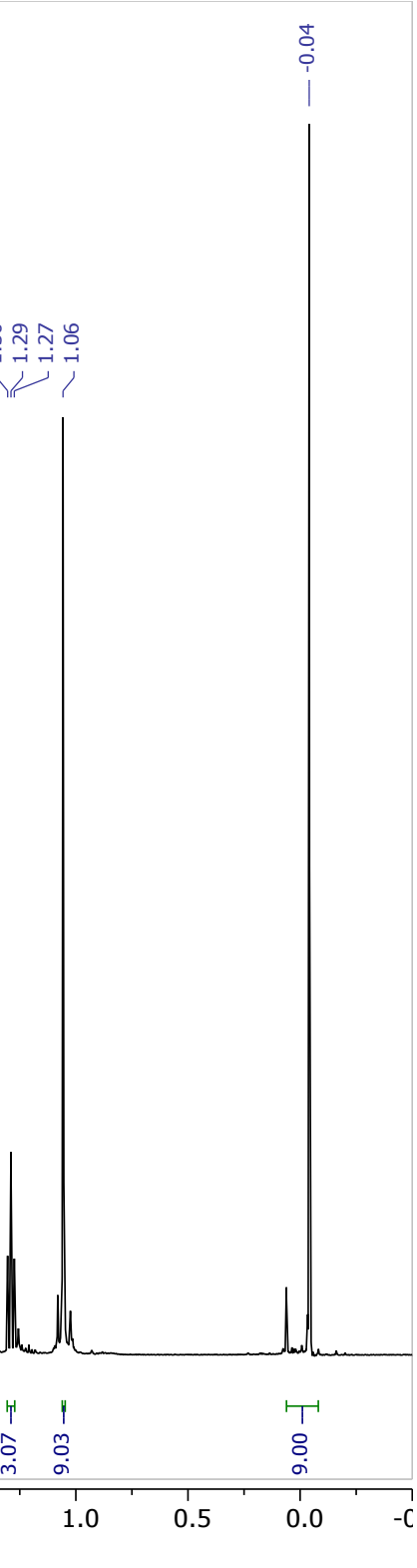



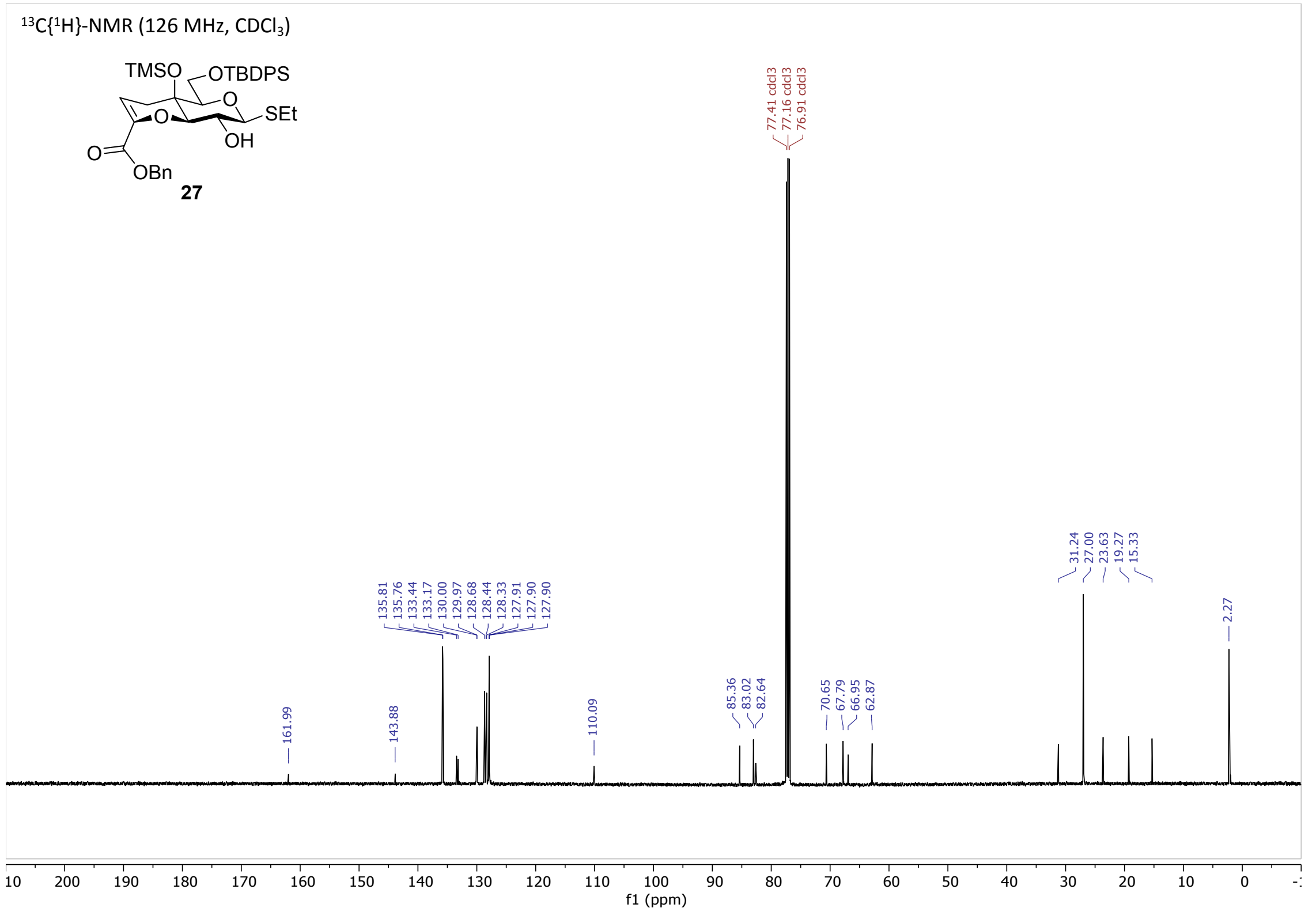
${ }^{1} \mathrm{H}-\mathrm{NMR}\left(500 \mathrm{MHz}, \mathrm{CDCl}_{3}\right.$ )

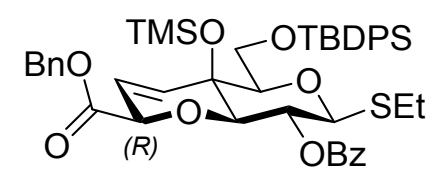

30a

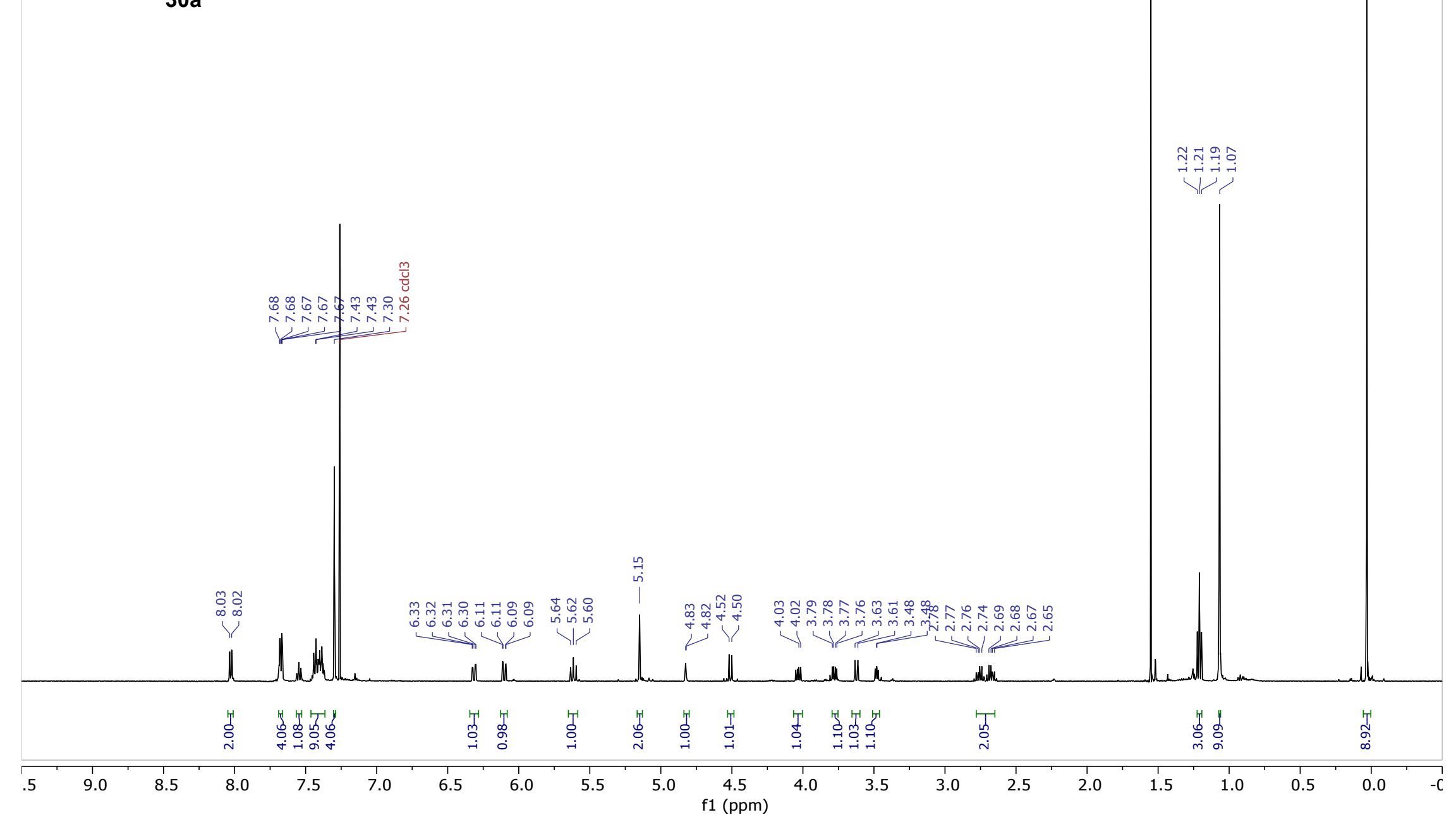


${ }^{13} \mathrm{C}\left\{{ }^{1} \mathrm{H}\right\}-\mathrm{NMR}\left(126 \mathrm{MHz}, \mathrm{CDCl}_{3}\right)$

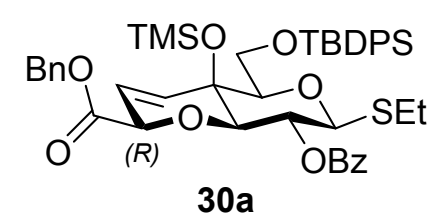

$30 a$

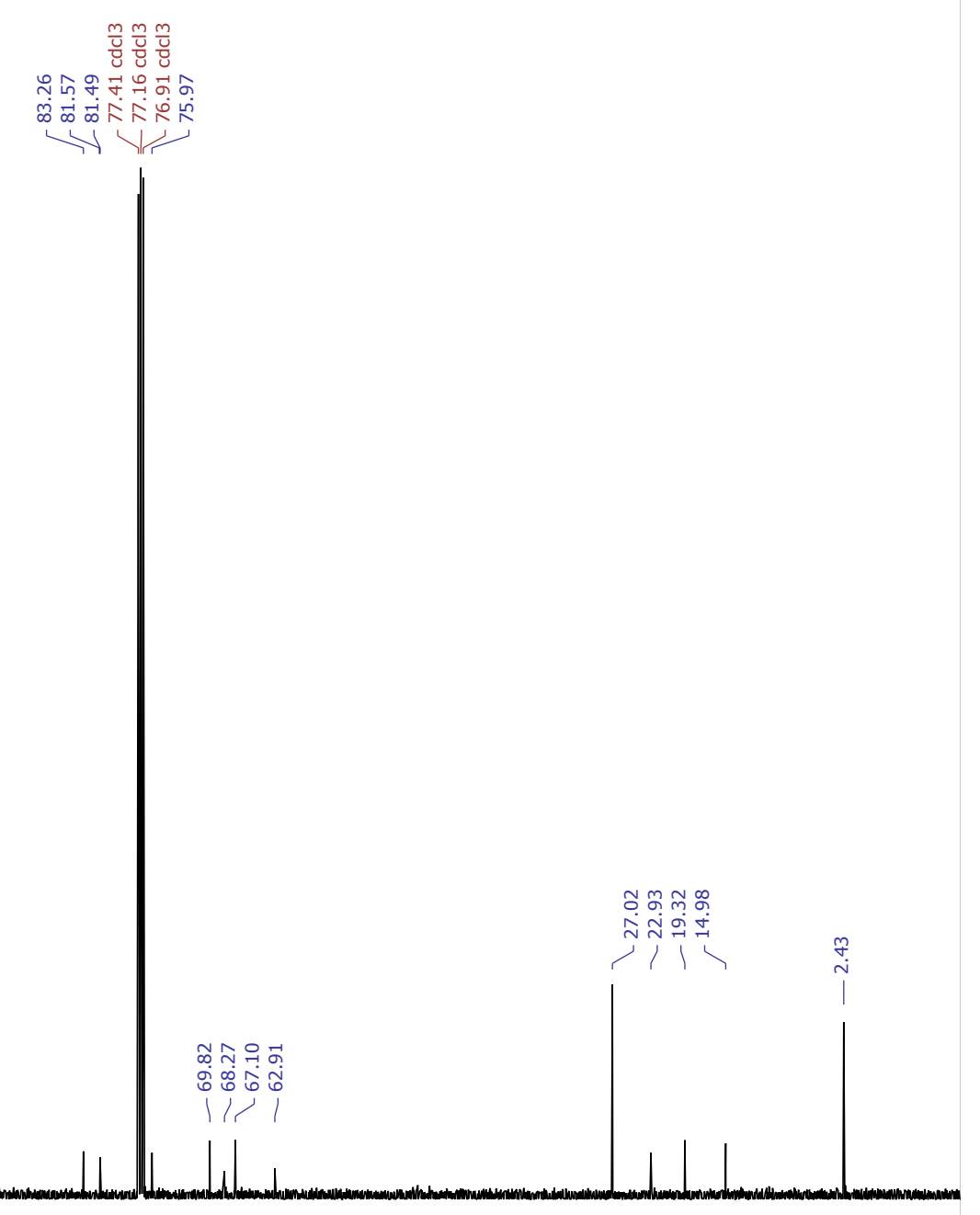

\begin{tabular}{lllll}
\hline 10 & 200 & 190 & 180 & 170
\end{tabular} 
${ }^{1} \mathrm{H}-\mathrm{NMR}\left(500 \mathrm{MHz}, \mathrm{CDCl}_{3}\right.$ )

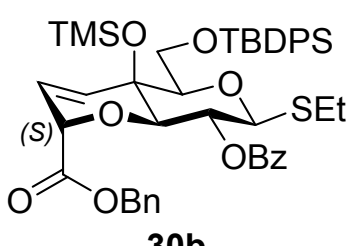

$30 \mathrm{~b}$

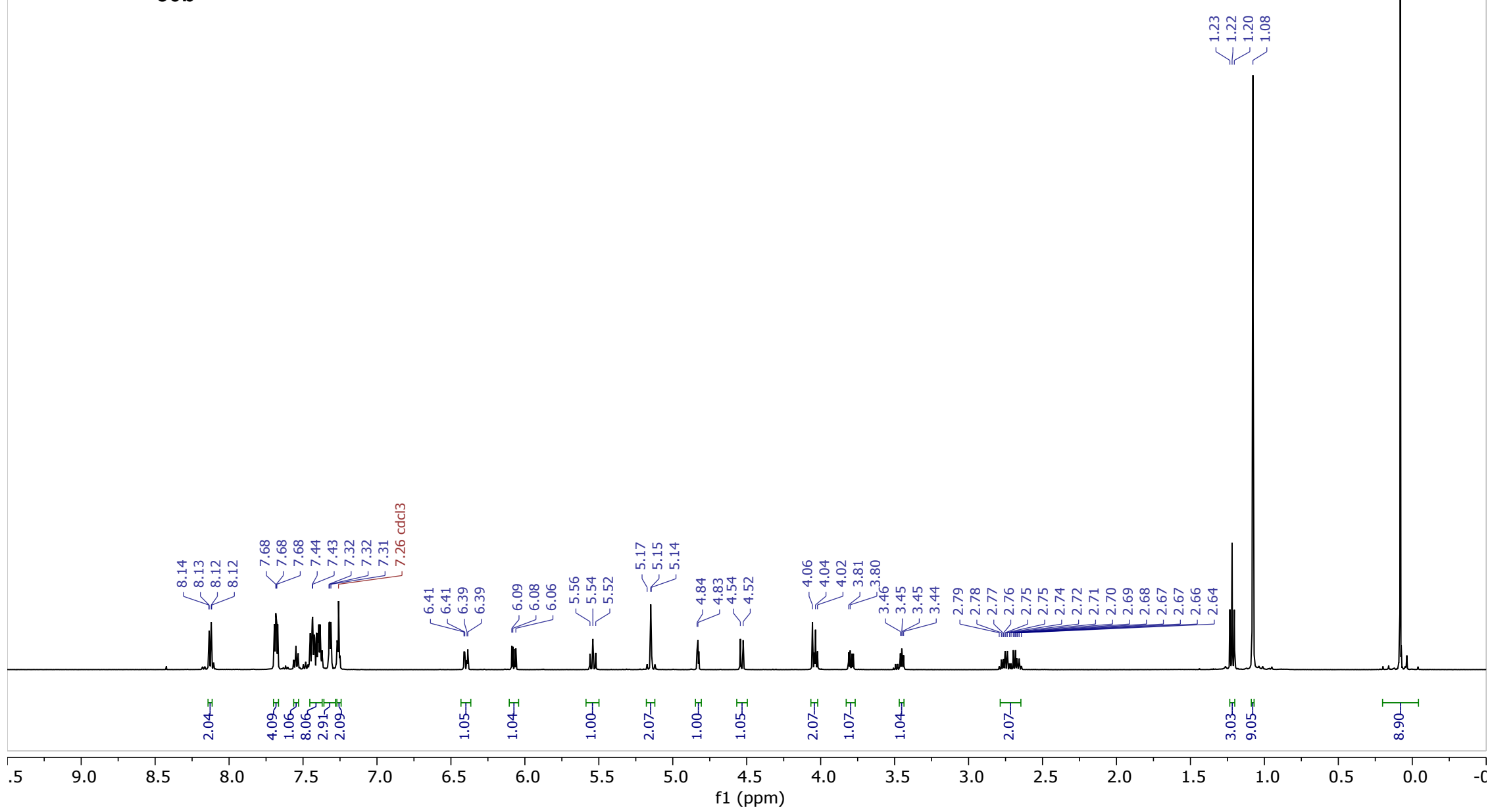


${ }^{13} \mathrm{C}\left\{{ }^{1} \mathrm{H}\right\}-\mathrm{NMR}\left(126 \mathrm{MHz}, \mathrm{CDCl}_{3}\right)$

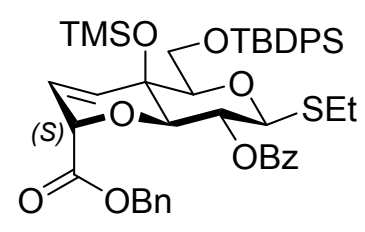

$30 \mathrm{~b}$

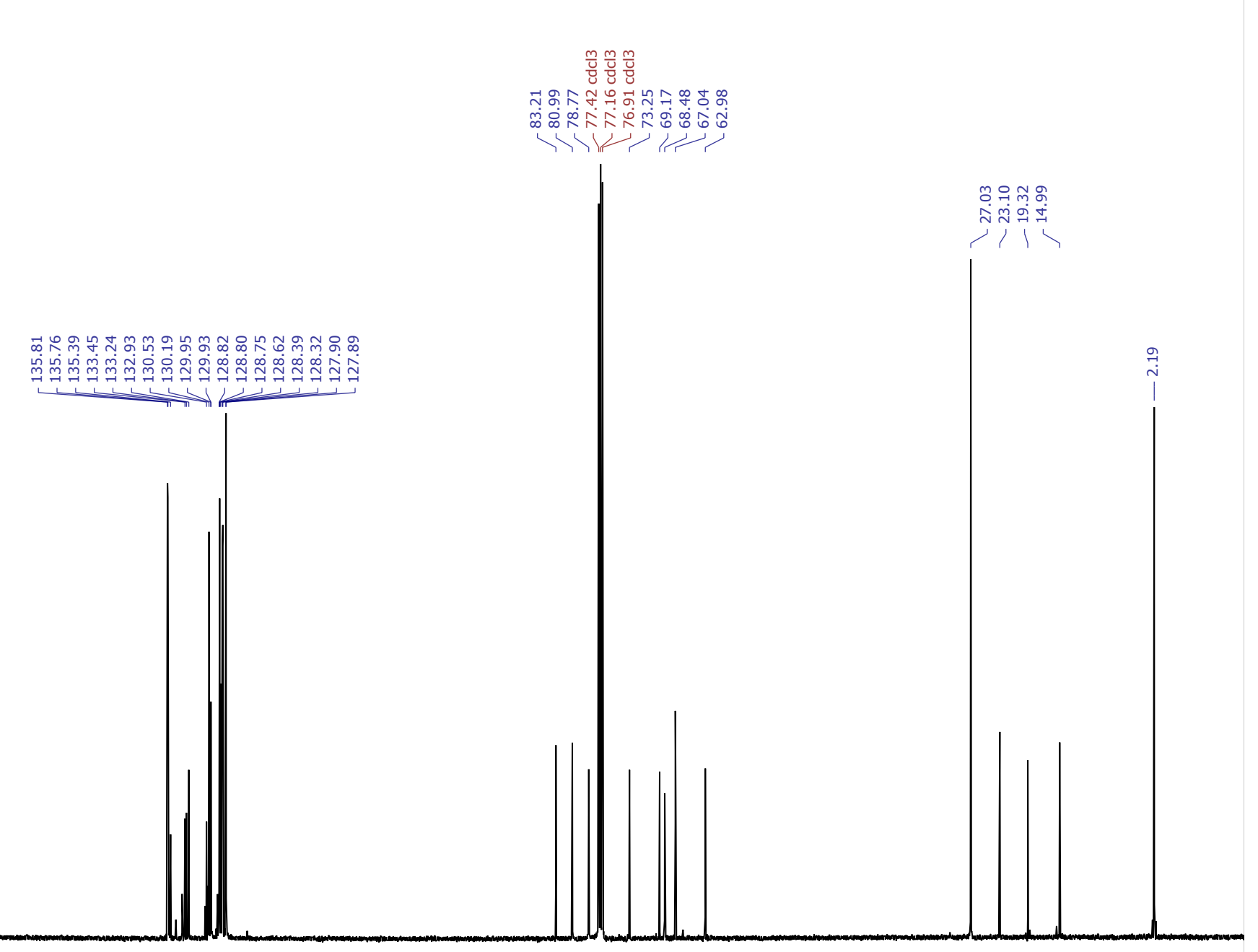

$10 \quad 200$ 190 180 160 $150 \quad 140$ 130 
${ }^{1} \mathrm{H}-\mathrm{NMR}\left(500 \mathrm{MHz}, \mathrm{CDCl}_{3}\right)$

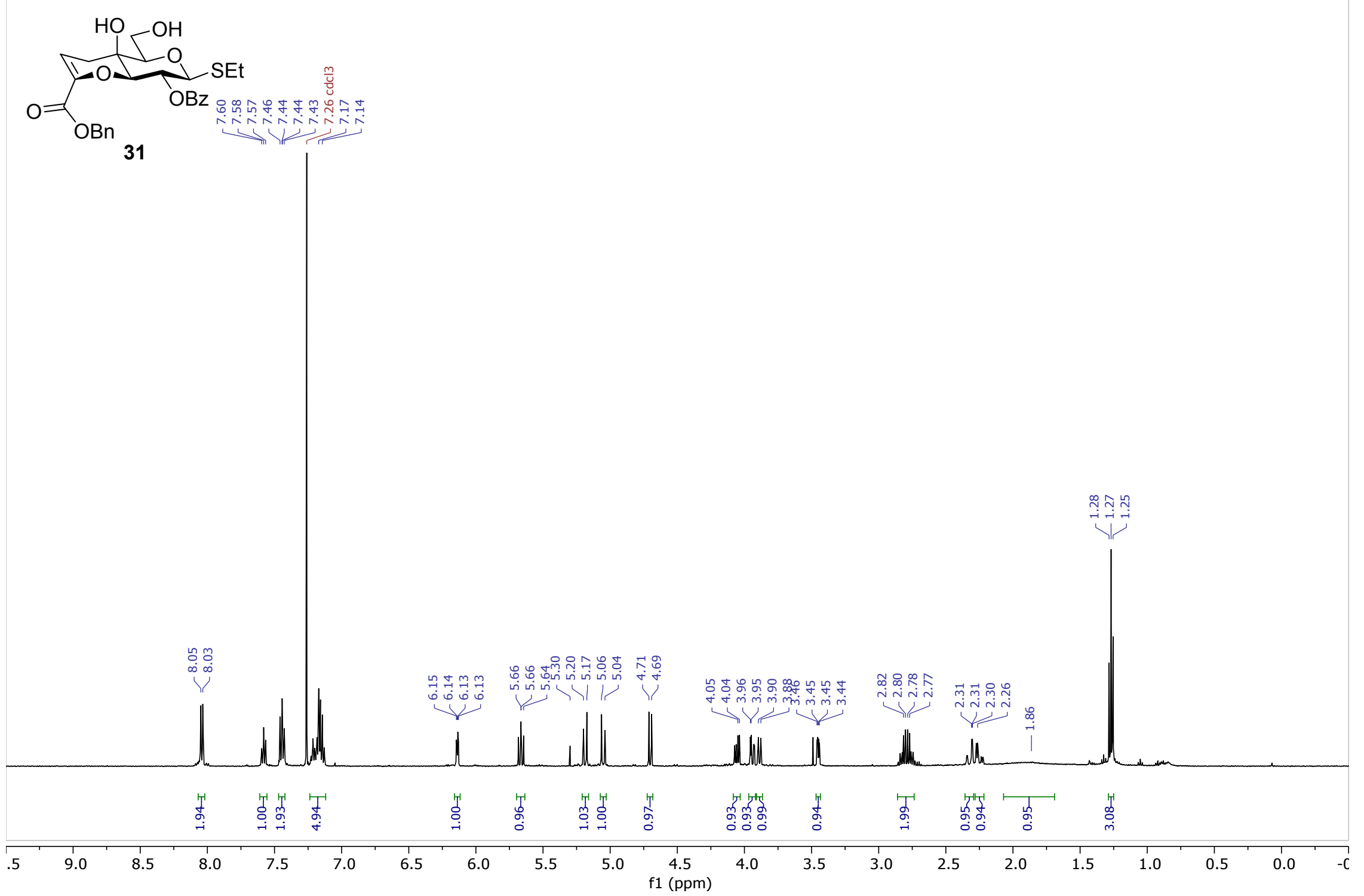


${ }^{13} \mathrm{C}\left\{{ }^{1} \mathrm{H}\right\}-\mathrm{NMR}\left(126 \mathrm{MHz}, \mathrm{CDCl}_{3}\right)$

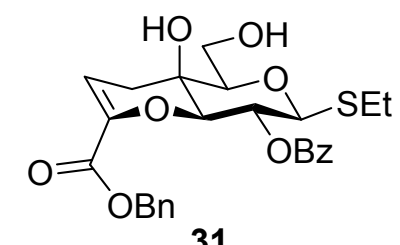

31

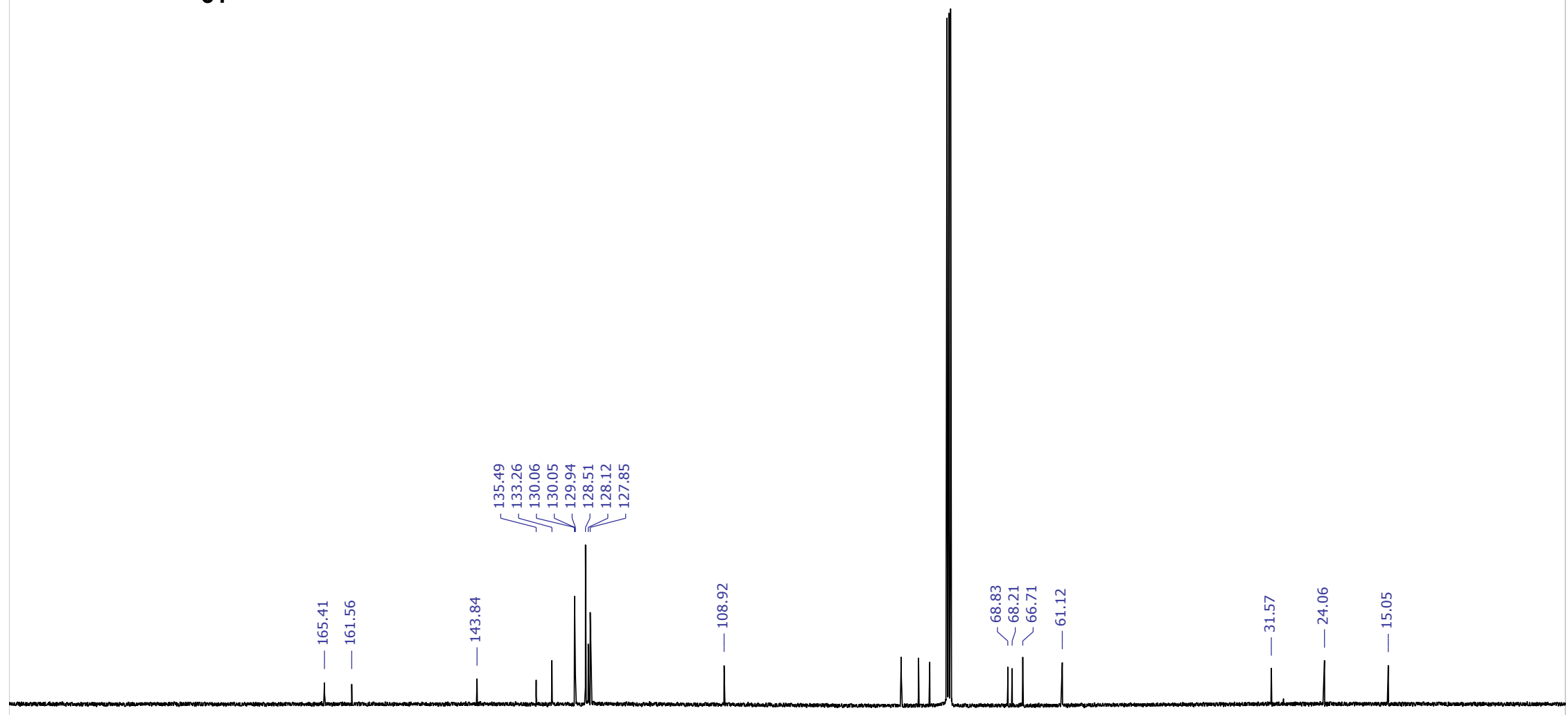

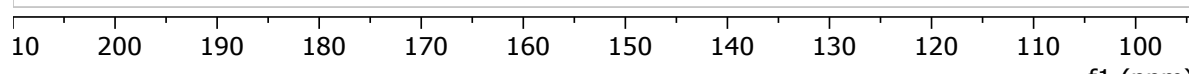
f1 (ppm)

$90 \quad 80$ $\begin{array}{ll}10 & 1 \\ 70 & \end{array}$ $60 \quad 50$ 40 30 


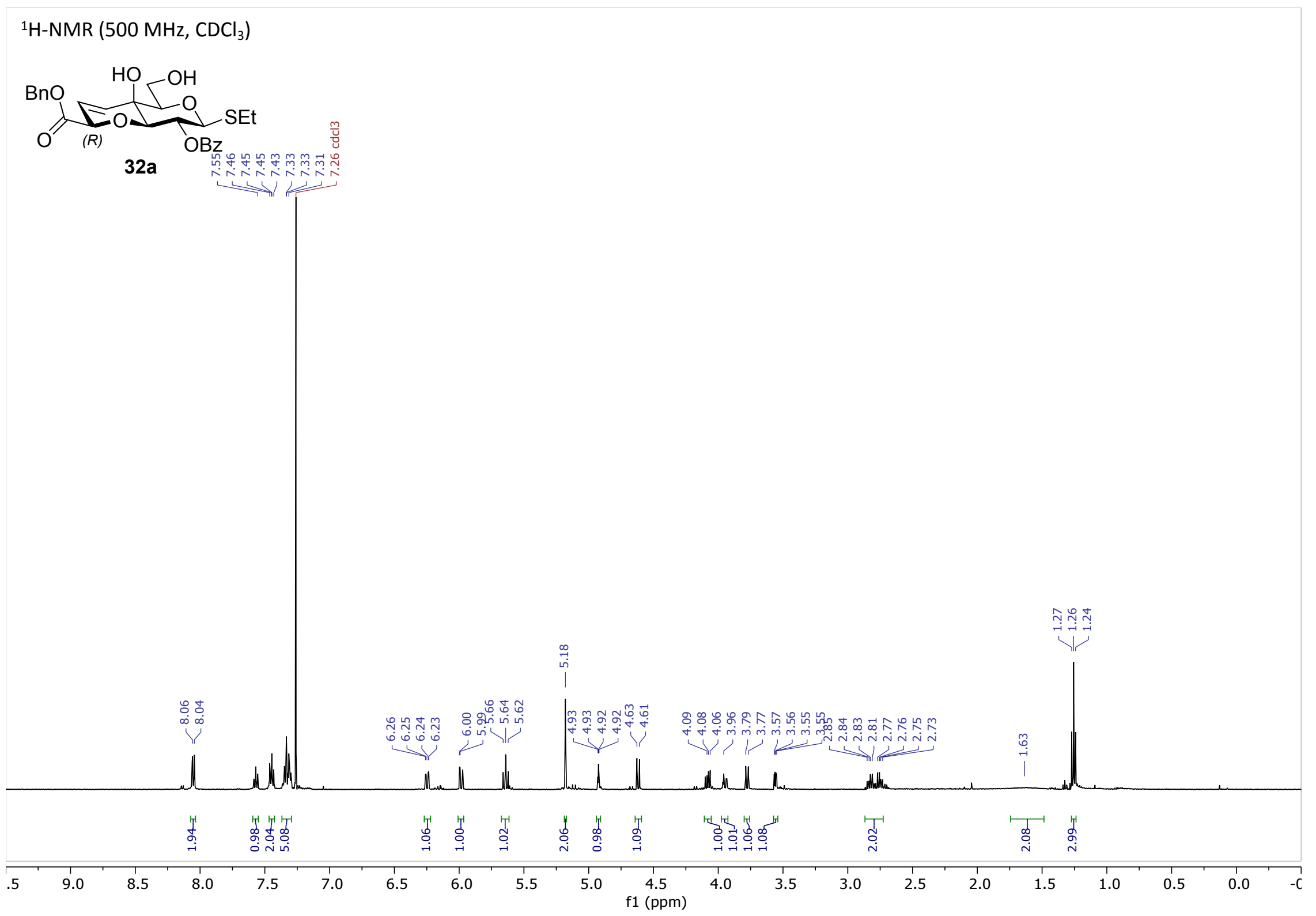


${ }^{13} \mathrm{C}\left\{{ }^{1} \mathrm{H}\right\}-\mathrm{NMR}\left(126 \mathrm{MHz}, \mathrm{CDCl}_{3}\right)$

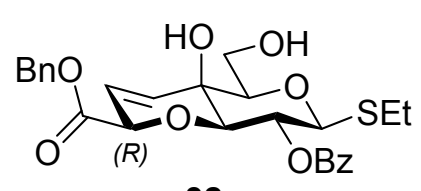

$32 a$

$\mathrm{OBz}^{\mathrm{O}} \mathrm{S}$

a

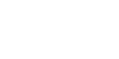
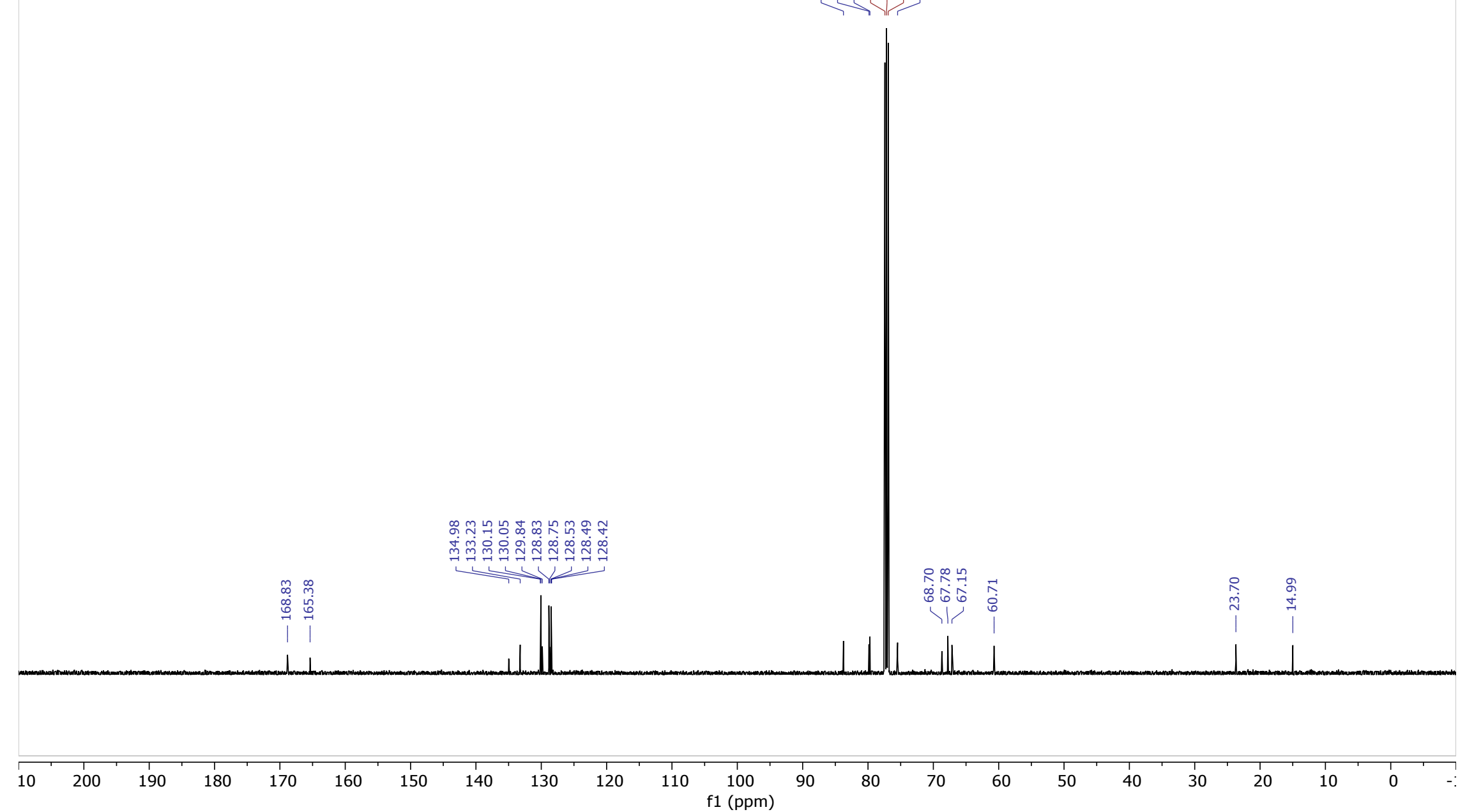
${ }^{1} \mathrm{H}-\mathrm{NMR}\left(500 \mathrm{MHz}, \mathrm{CDCl}_{3}\right)$

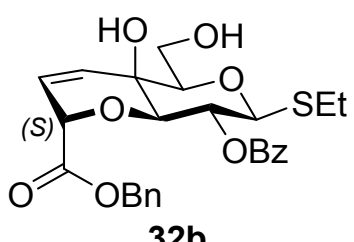

$32 b$

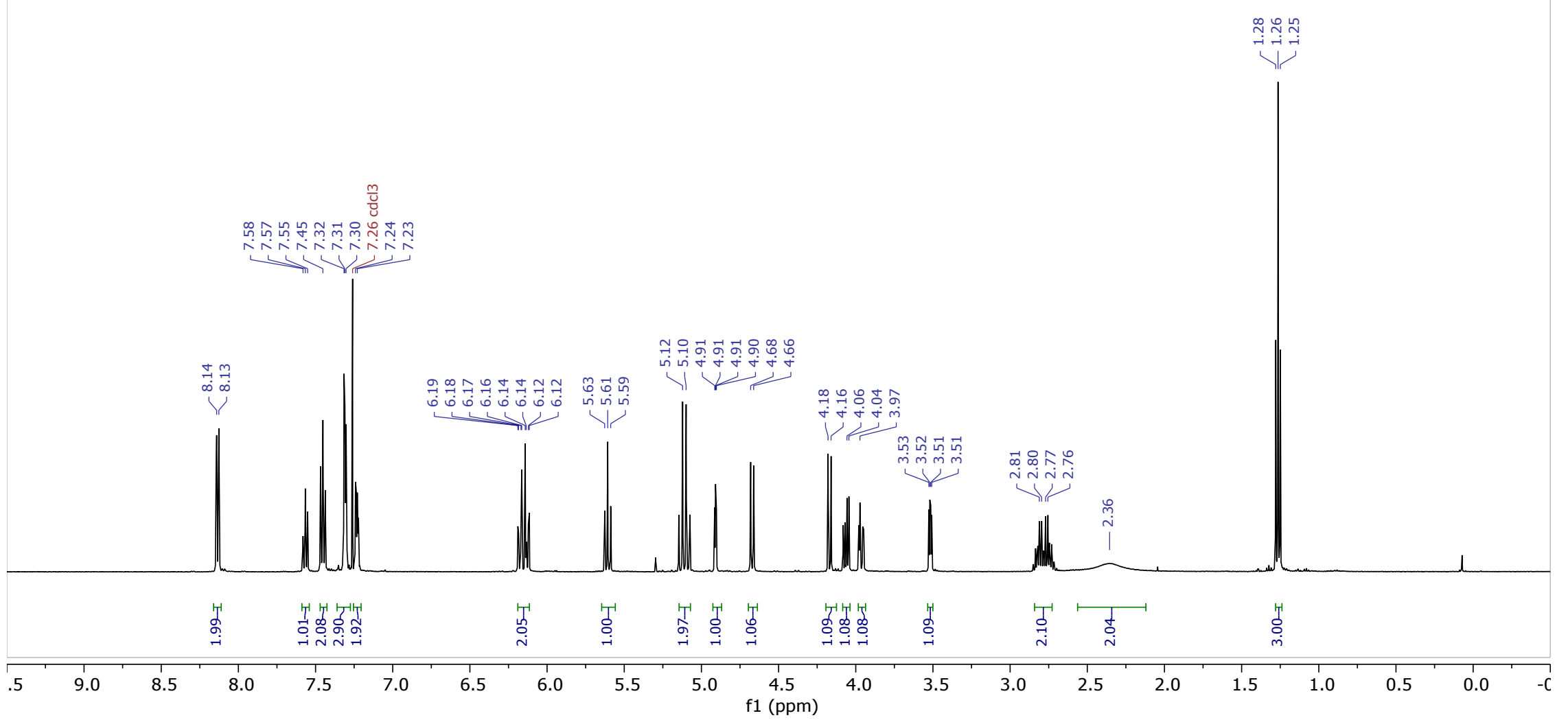


${ }^{13} \mathrm{C}\left\{{ }^{1} \mathrm{H}\right\}-\mathrm{NMR}\left(126 \mathrm{MHz}, \mathrm{CDCl}_{3}\right.$ )

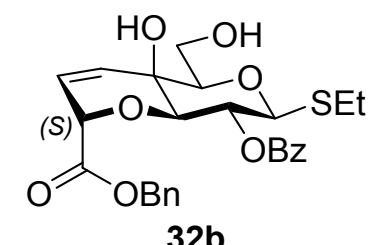

$32 b$
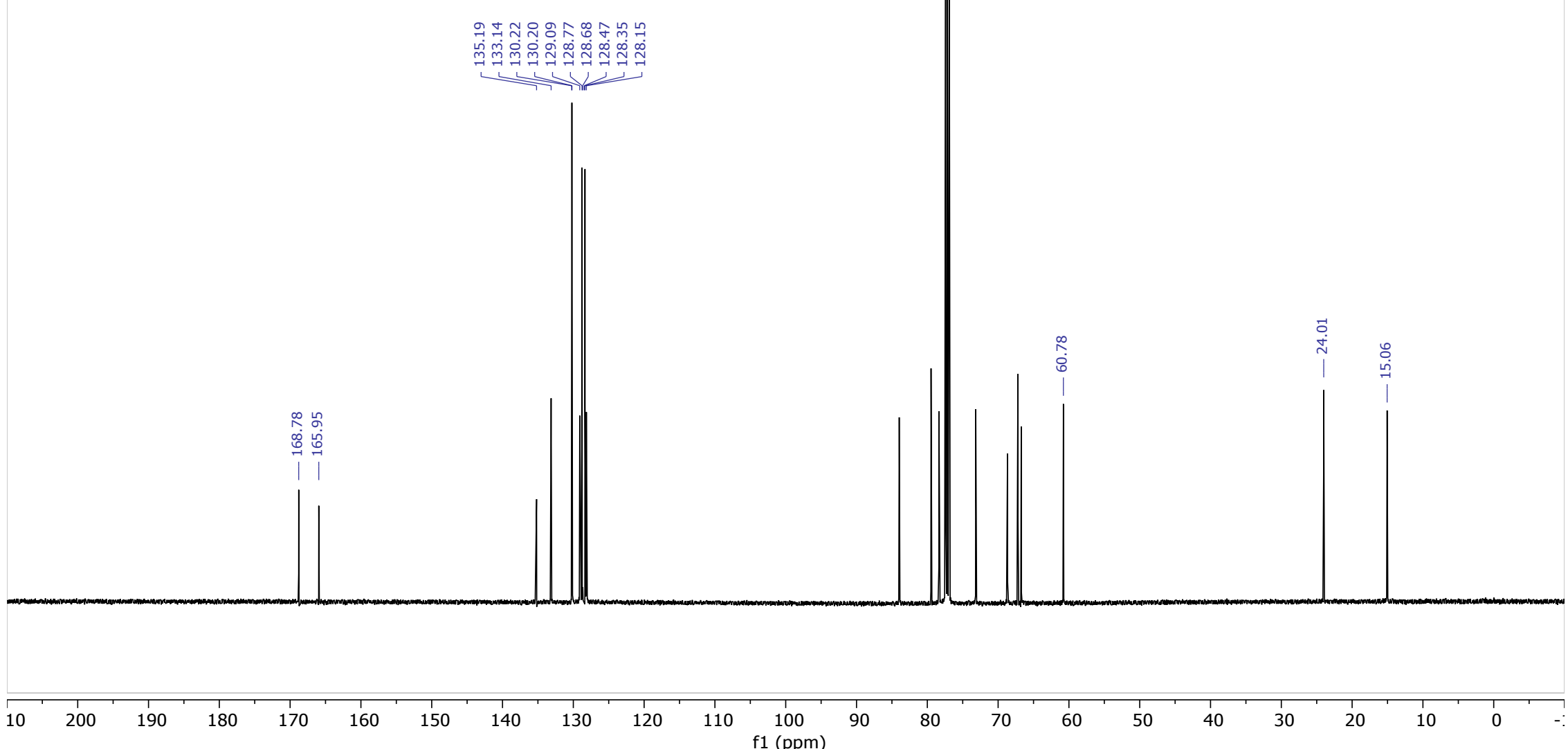


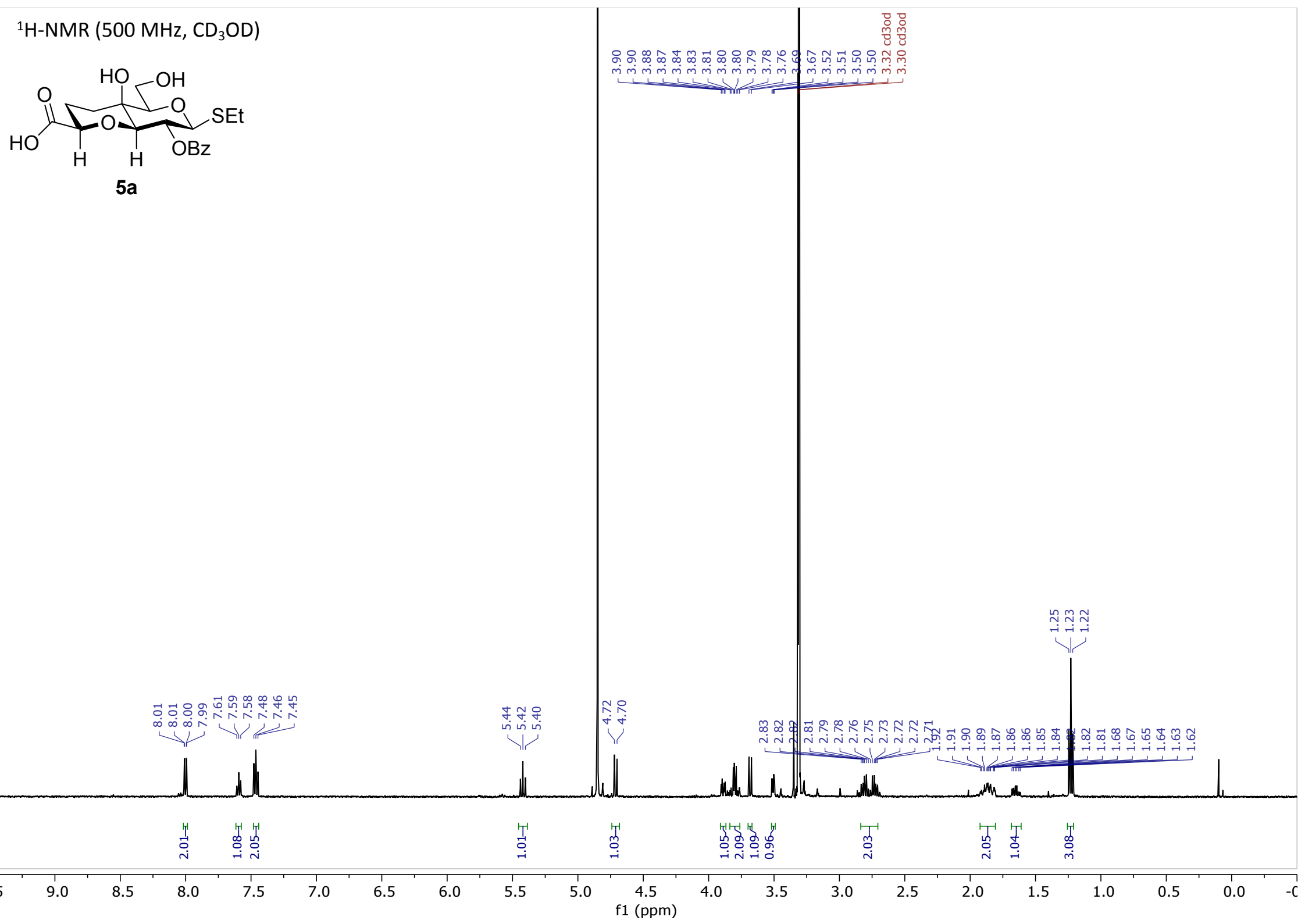




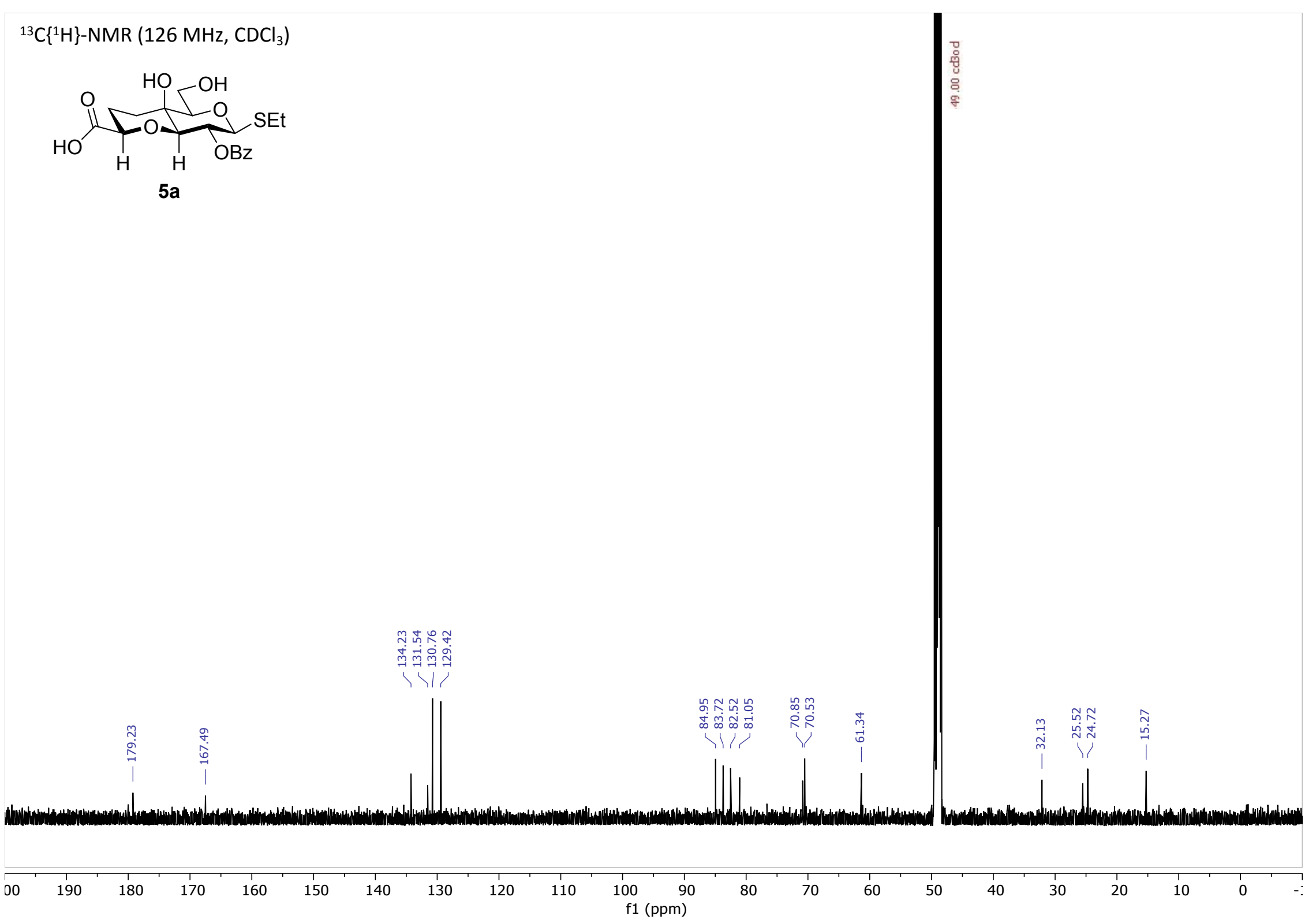


${ }^{1} \mathrm{H}-\mathrm{NMR}\left(500 \mathrm{MHz}, \mathrm{CD}_{3} \mathrm{OD}\right)$

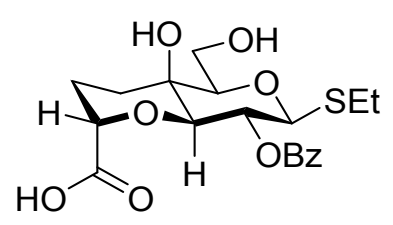

$5 b$

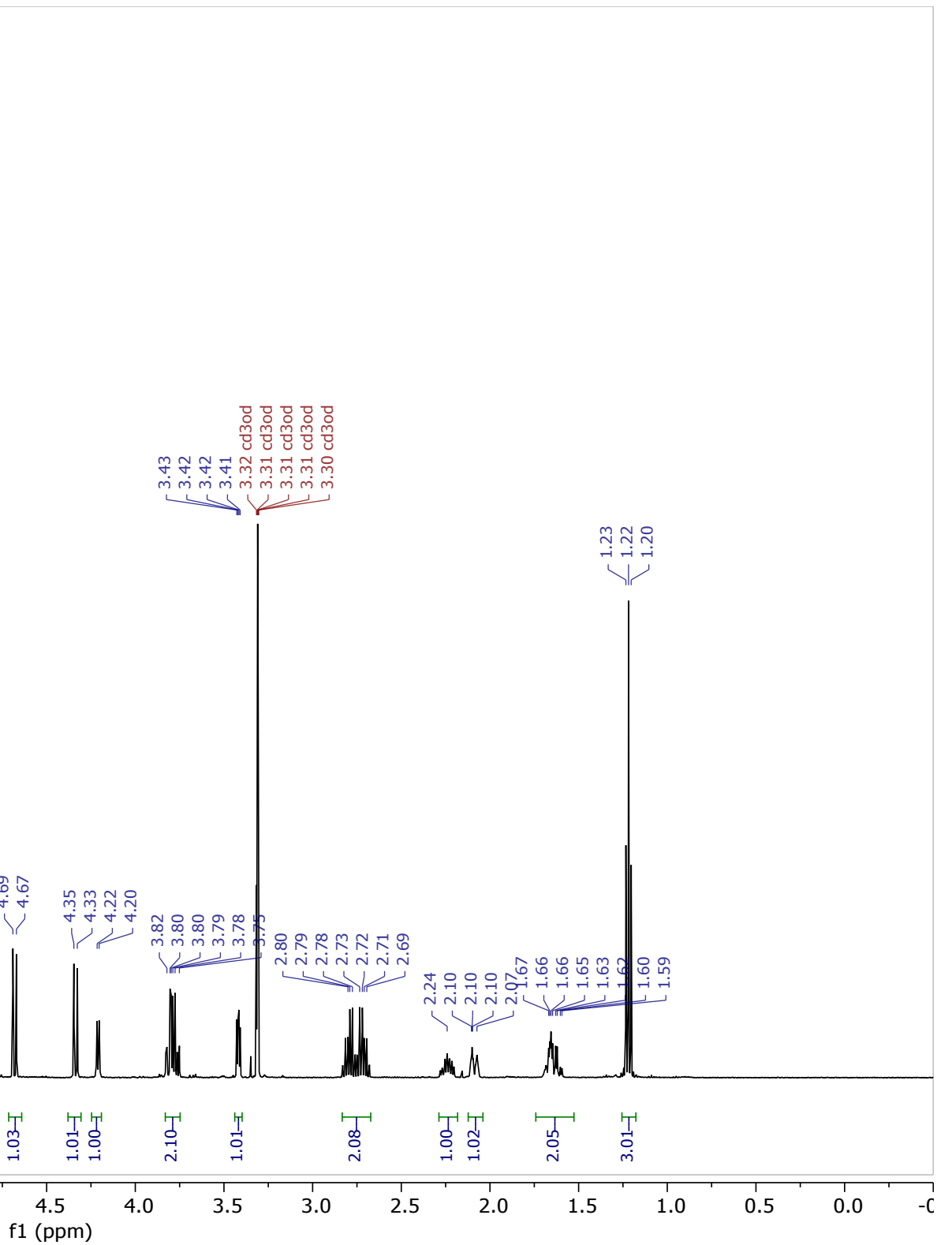


${ }^{13} \mathrm{C}\left\{{ }^{1} \mathrm{H}\right\}-N M R\left(126 \mathrm{MHz}, \mathrm{CD}_{3} \mathrm{OD}\right)$

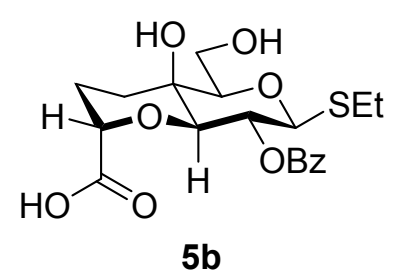

$5 b$

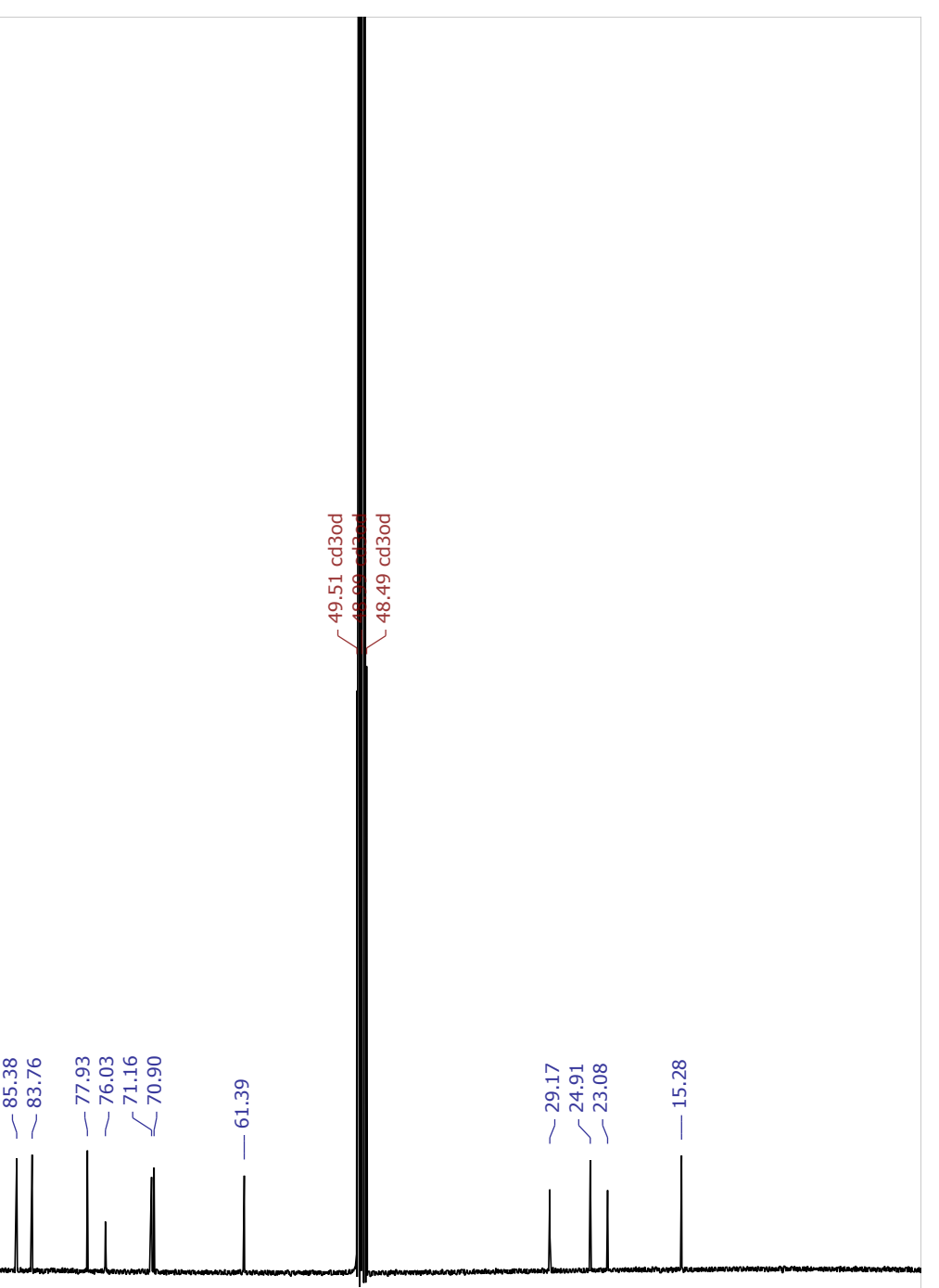

10

200

190

180

160

150

140

130

120

$110 \quad 100$

$90 \quad 80$

70

$60 \quad 50$

40

$30 \quad 20$

$10 \quad 0$ 
${ }^{1} \mathrm{H}-\mathrm{NMR}\left(500 \mathrm{MHz}, \mathrm{CDCl}_{3}\right)$

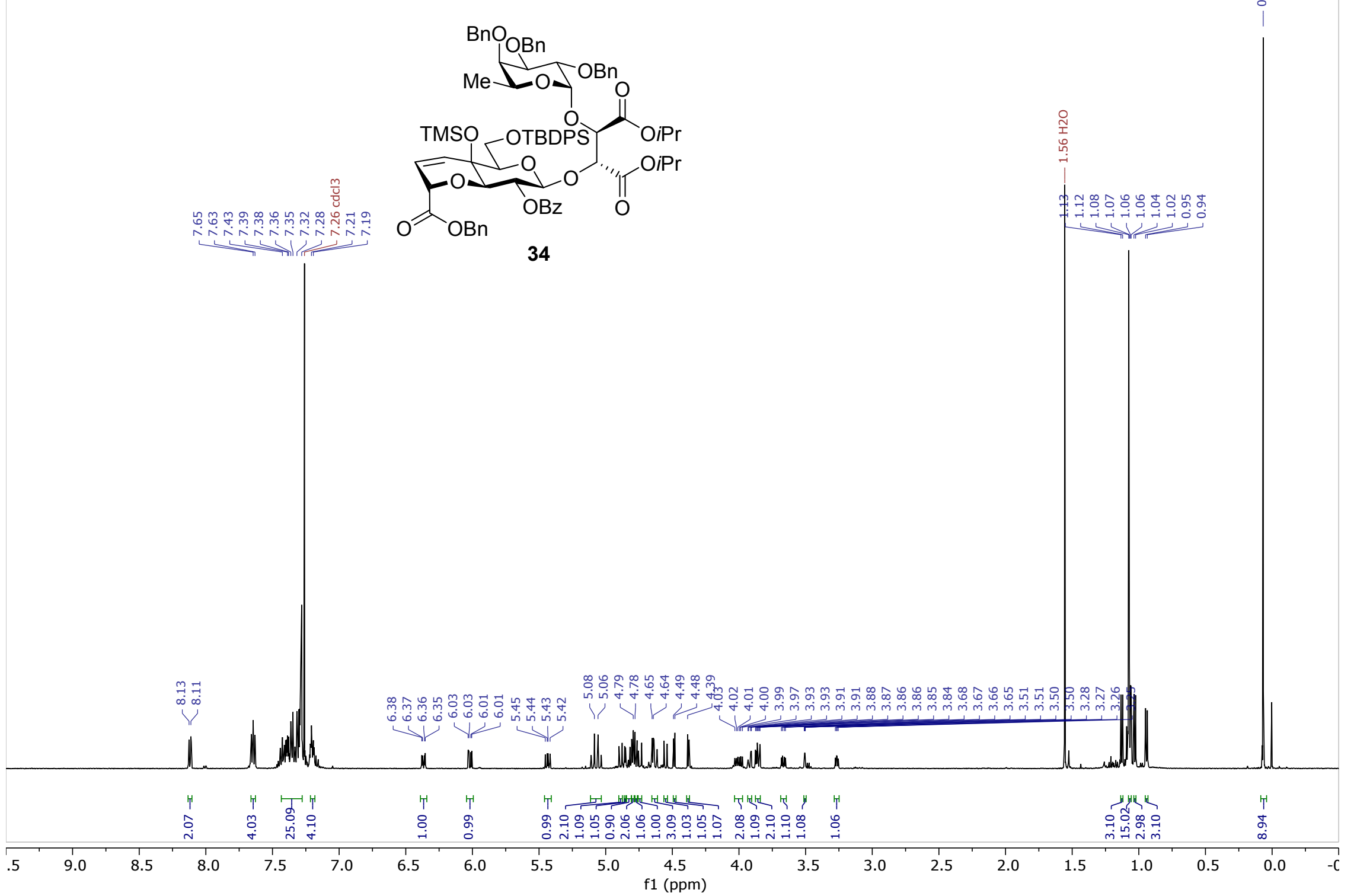


${ }^{13} \mathrm{C}\left\{{ }^{1} \mathrm{H}\right\}-\mathrm{NMR}\left(126 \mathrm{MHz}, \mathrm{CDCl}_{3}\right)$

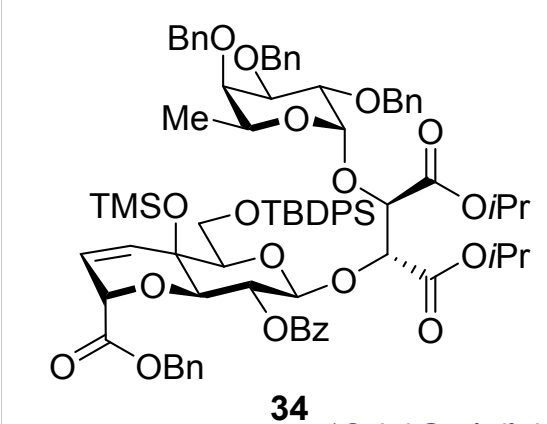

34

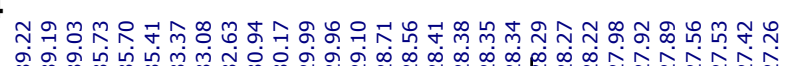

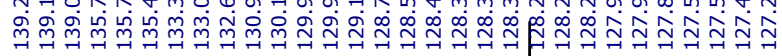

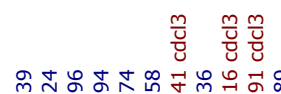

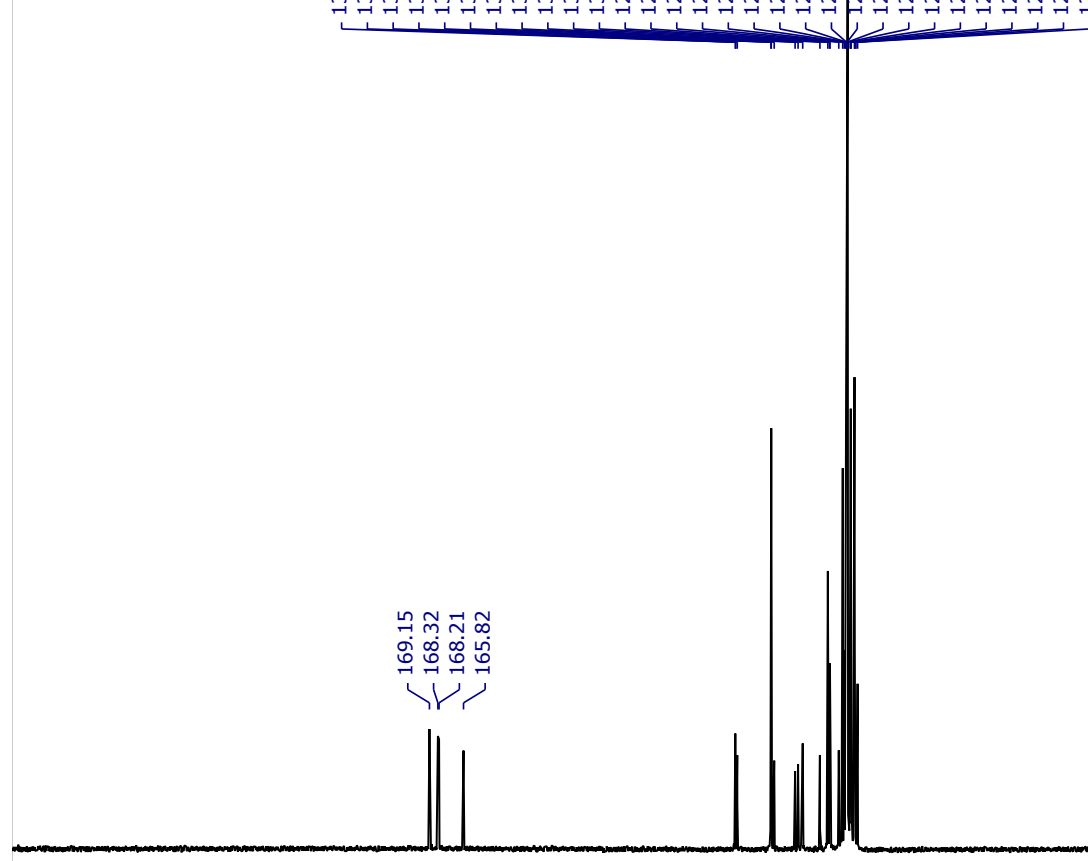

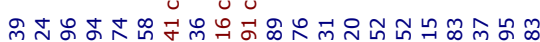

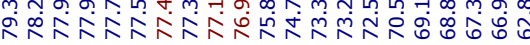

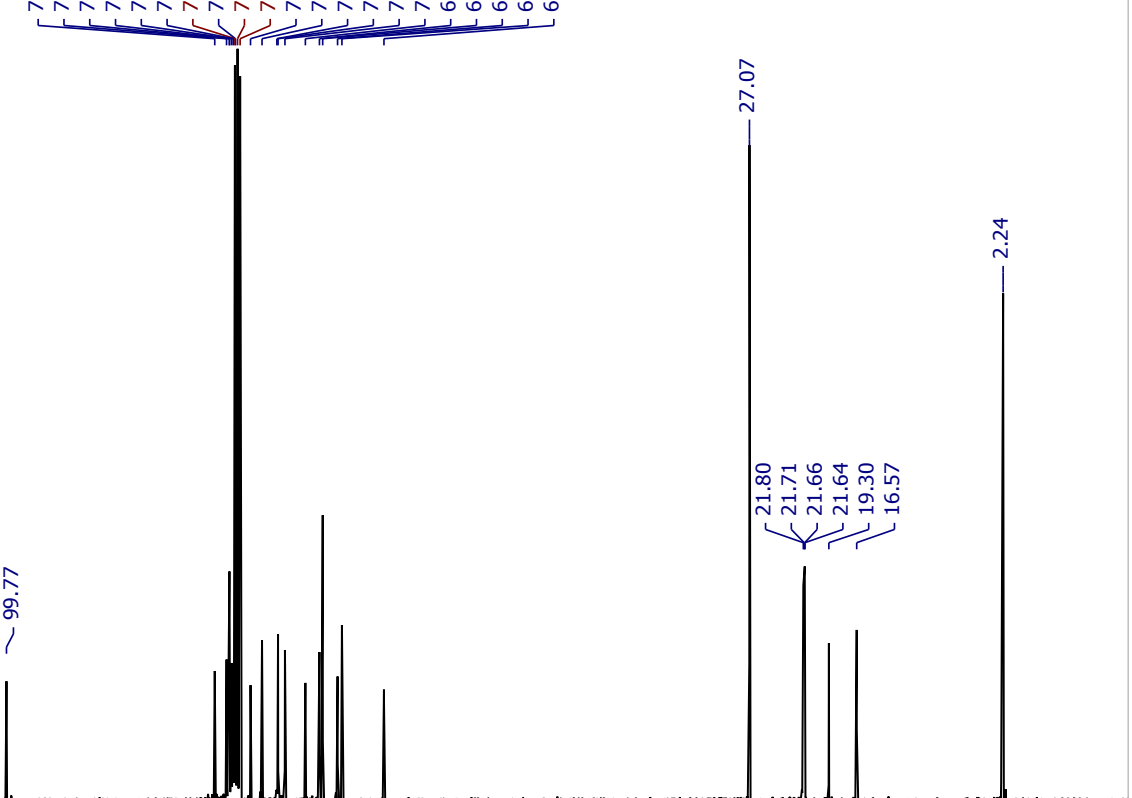

200

190

180

170

160

150

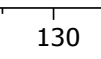

120

$110 \quad 100$

$90 \quad 80$

70

60

50

40

30

20

10 0 
${ }^{1} \mathrm{H}-\mathrm{NMR}\left(500 \mathrm{MHz}, \mathrm{CDCl}_{3}\right)$

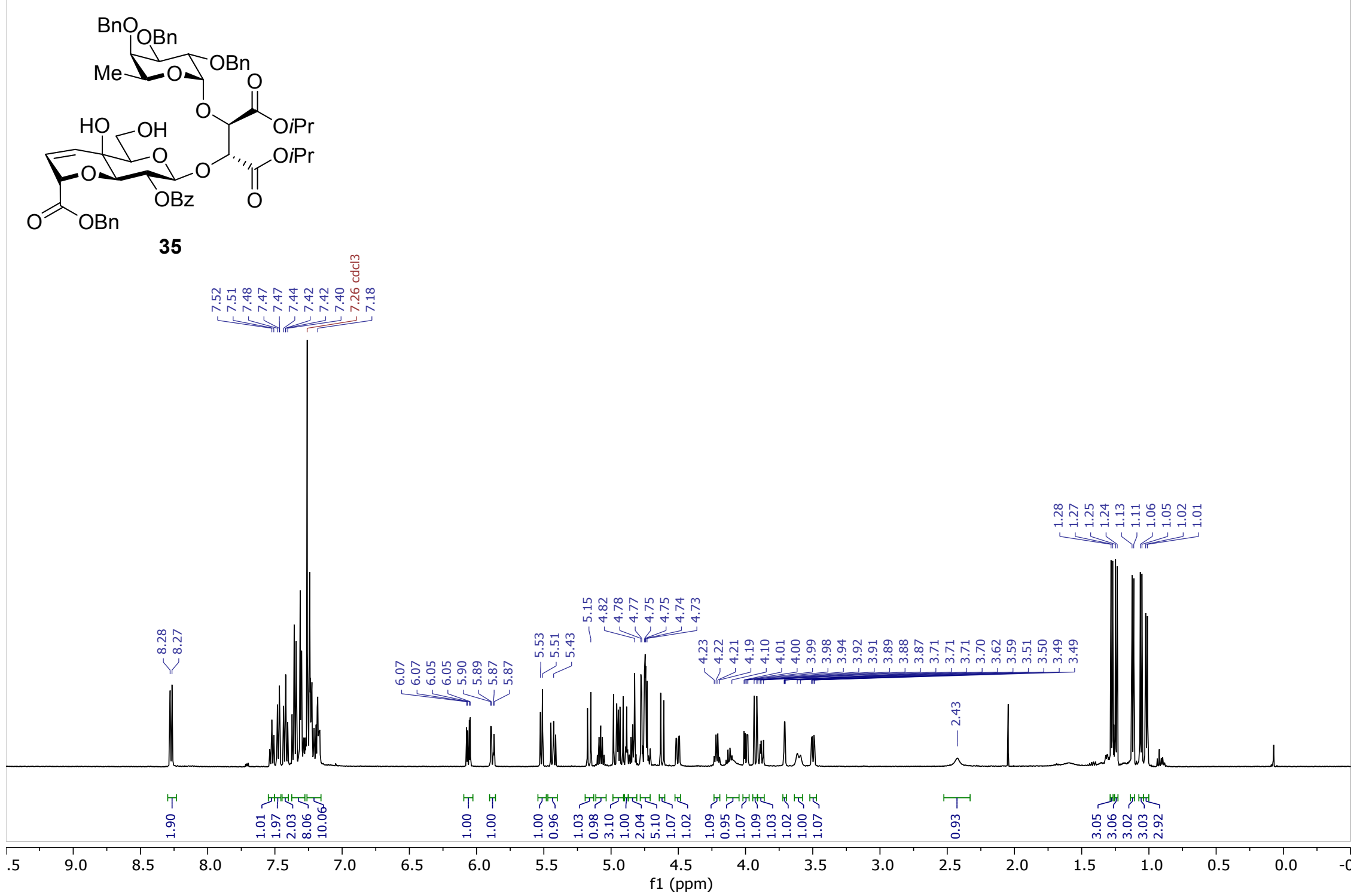


${ }^{13} \mathrm{C}\left\{{ }^{1} \mathrm{H}\right\}-\mathrm{NMR}\left(126 \mathrm{MHz}, \mathrm{CDCl}_{3}\right)$

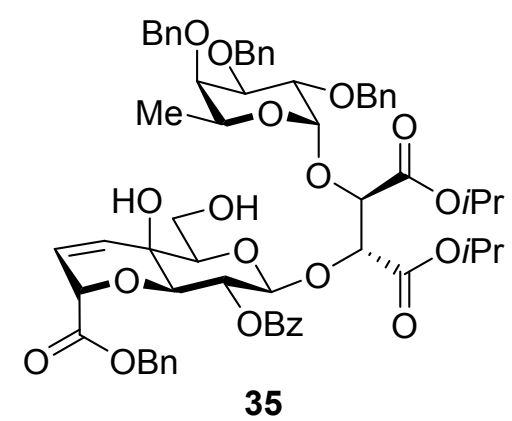

35

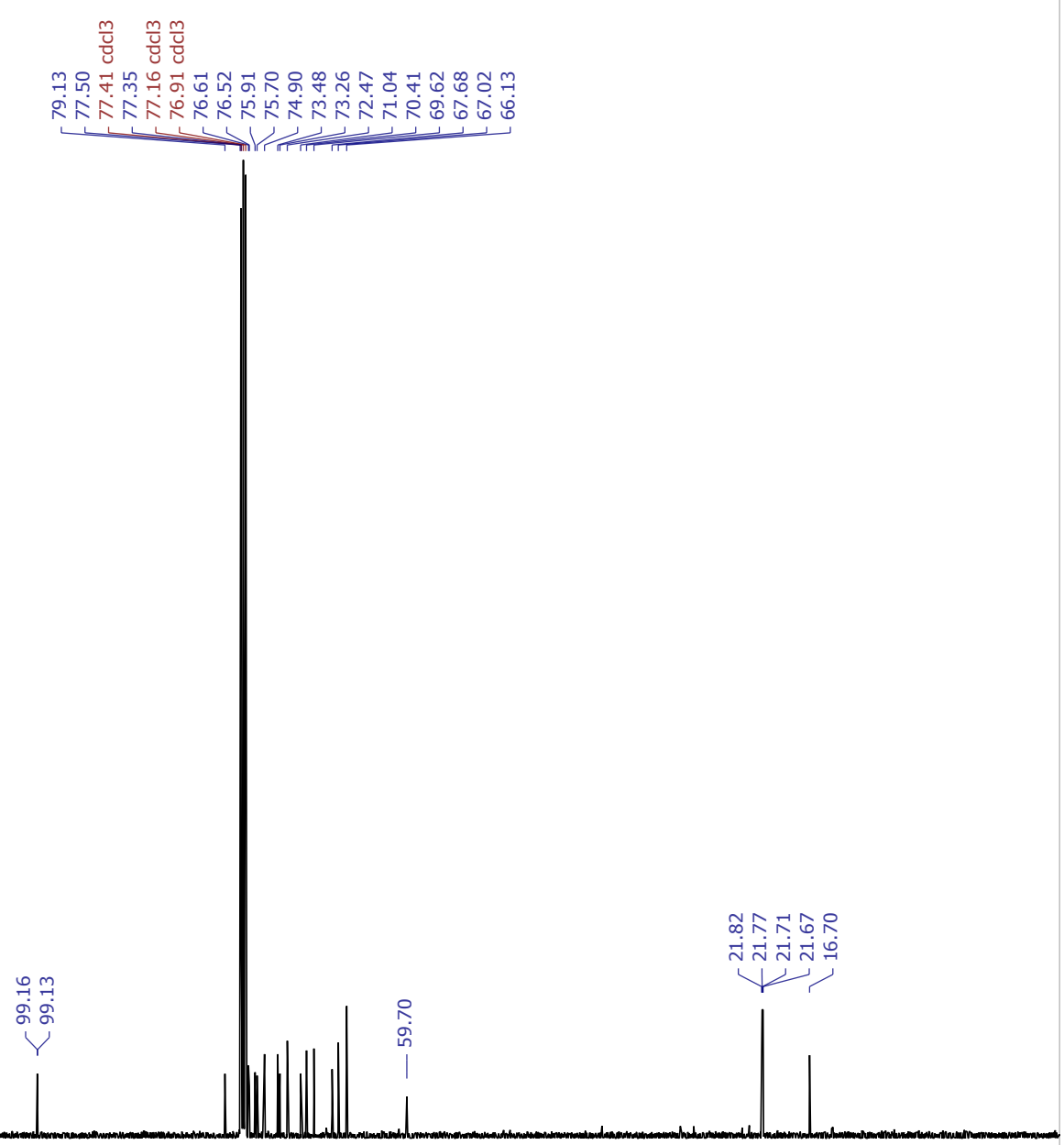

10

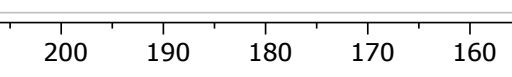

150
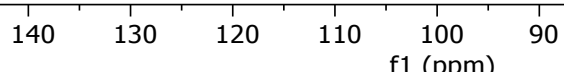

80

70

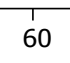

50

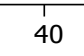


${ }^{1} \mathrm{H}-\mathrm{NMR}\left(500 \mathrm{MHz}, \mathrm{CDCl}_{3}\right.$ )

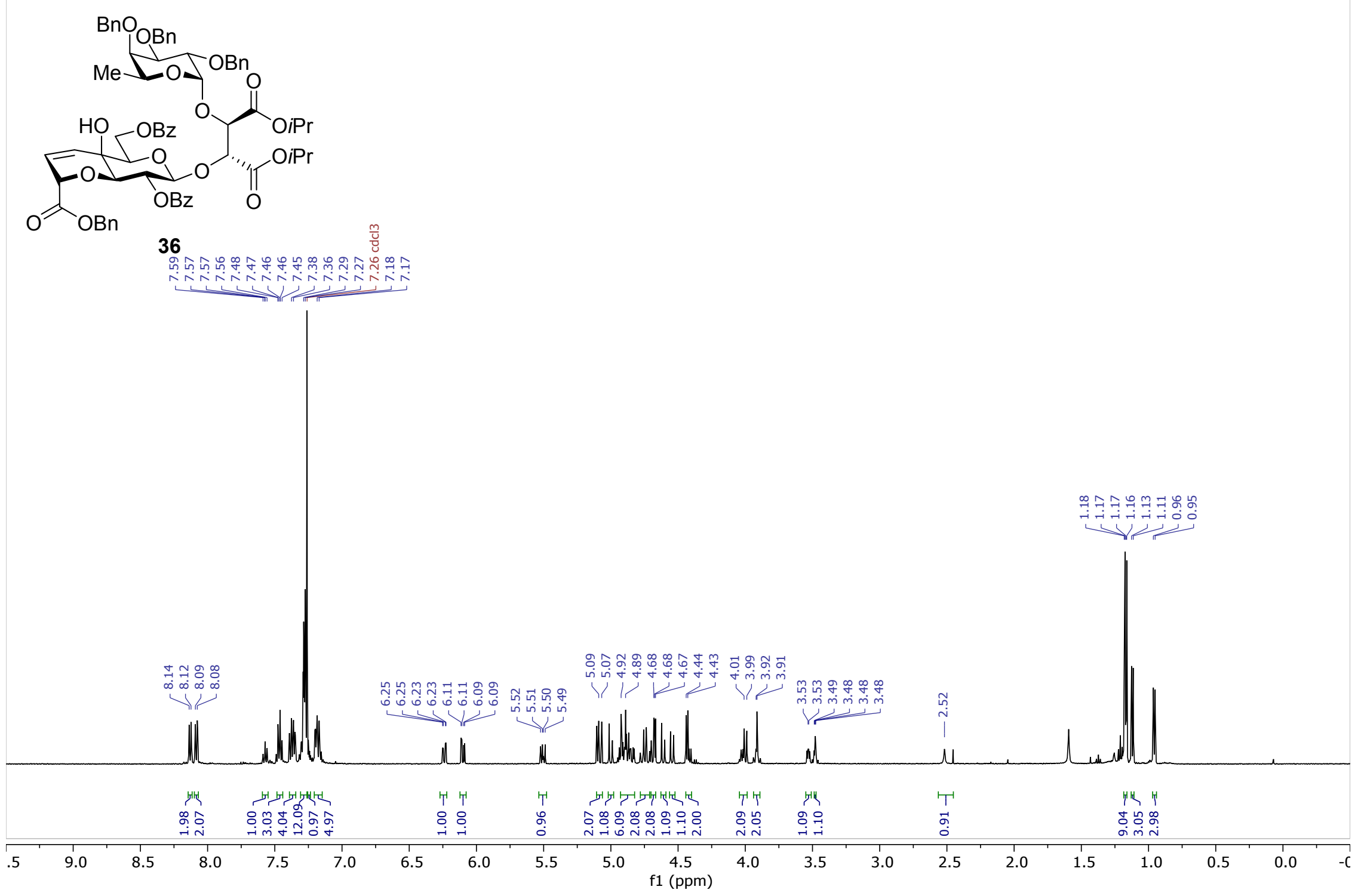




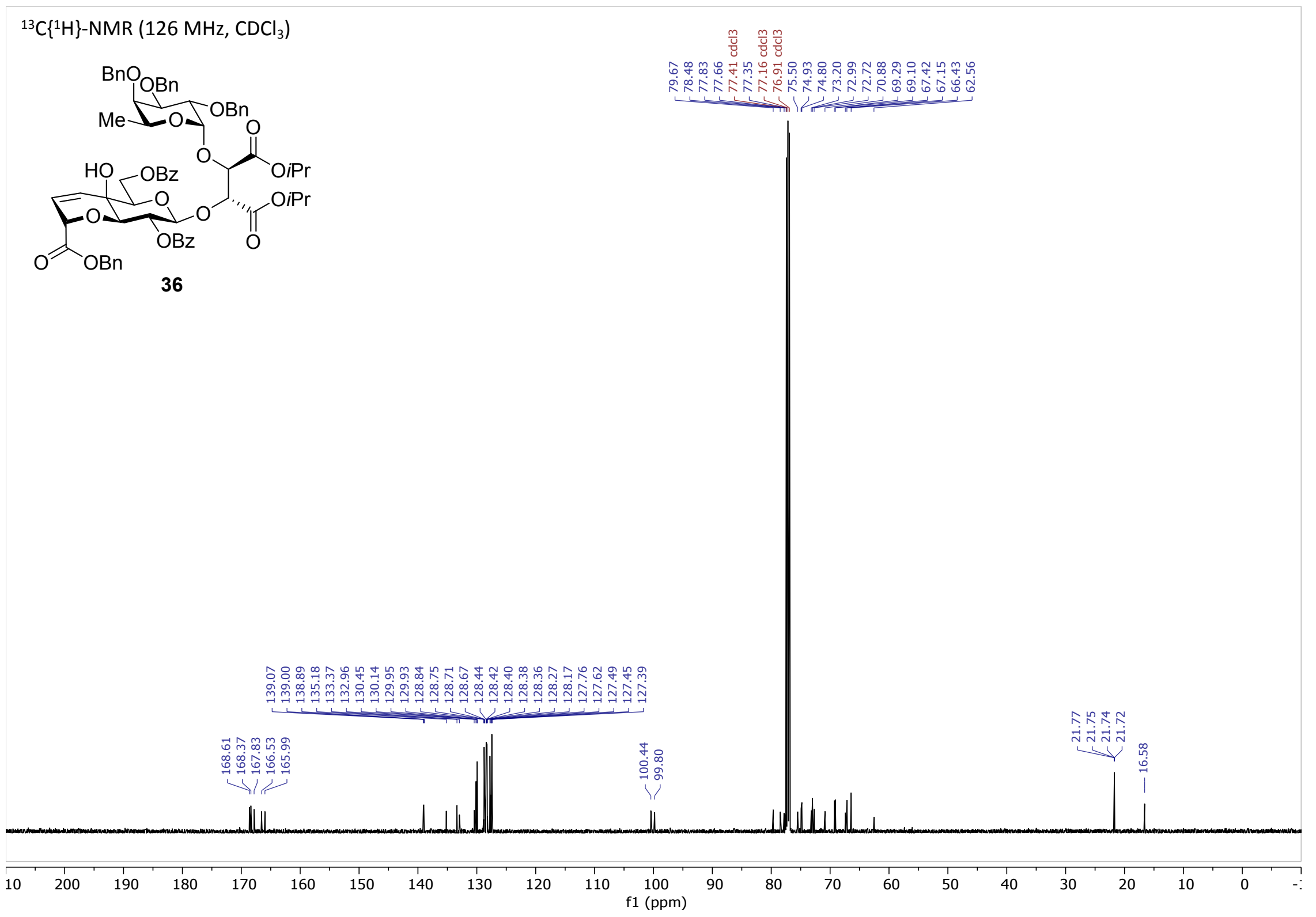


${ }^{1} \mathrm{H}-\mathrm{NMR}\left(500 \mathrm{MHz}, \mathrm{CDCl}_{3}\right.$ )

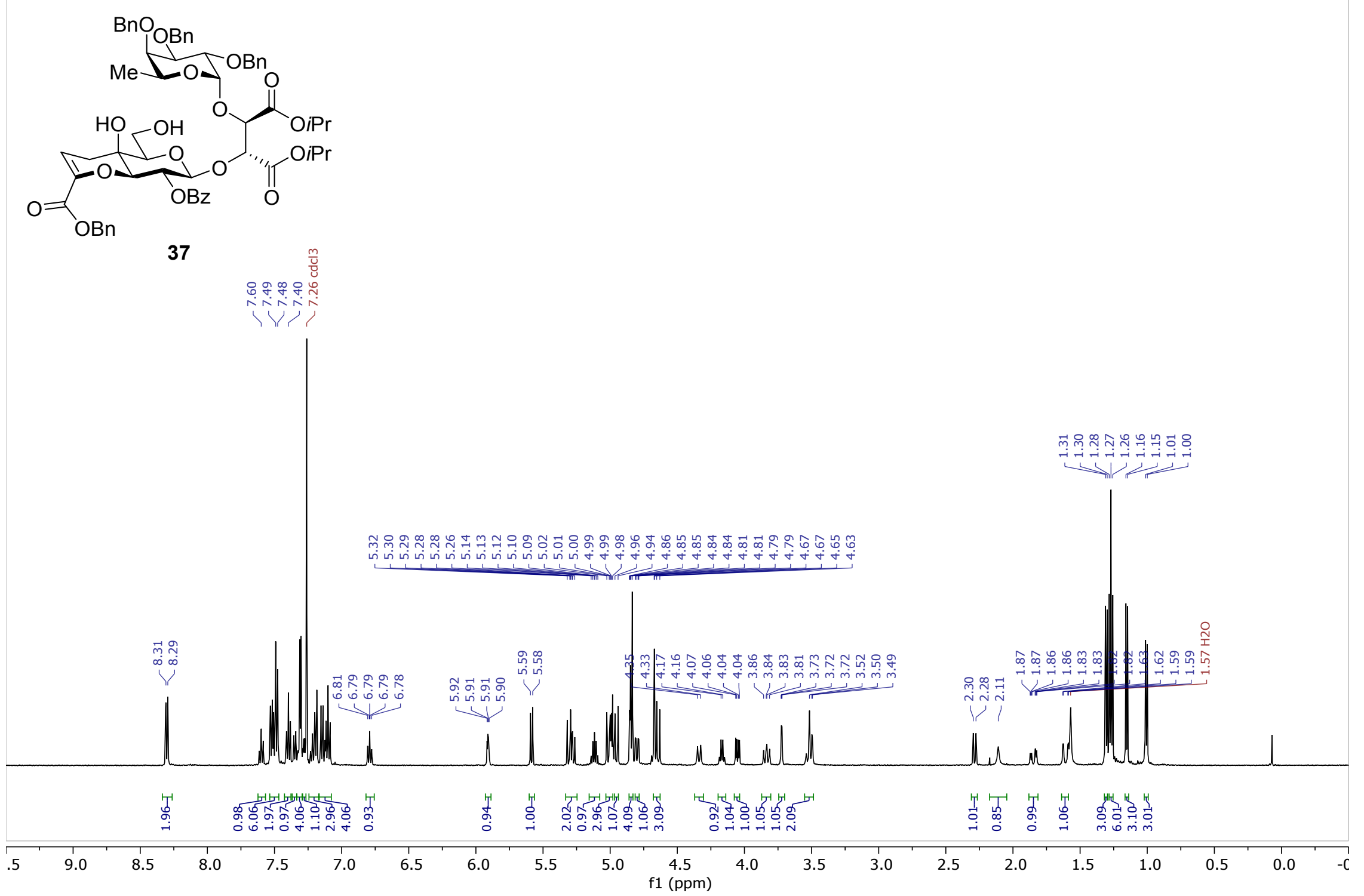




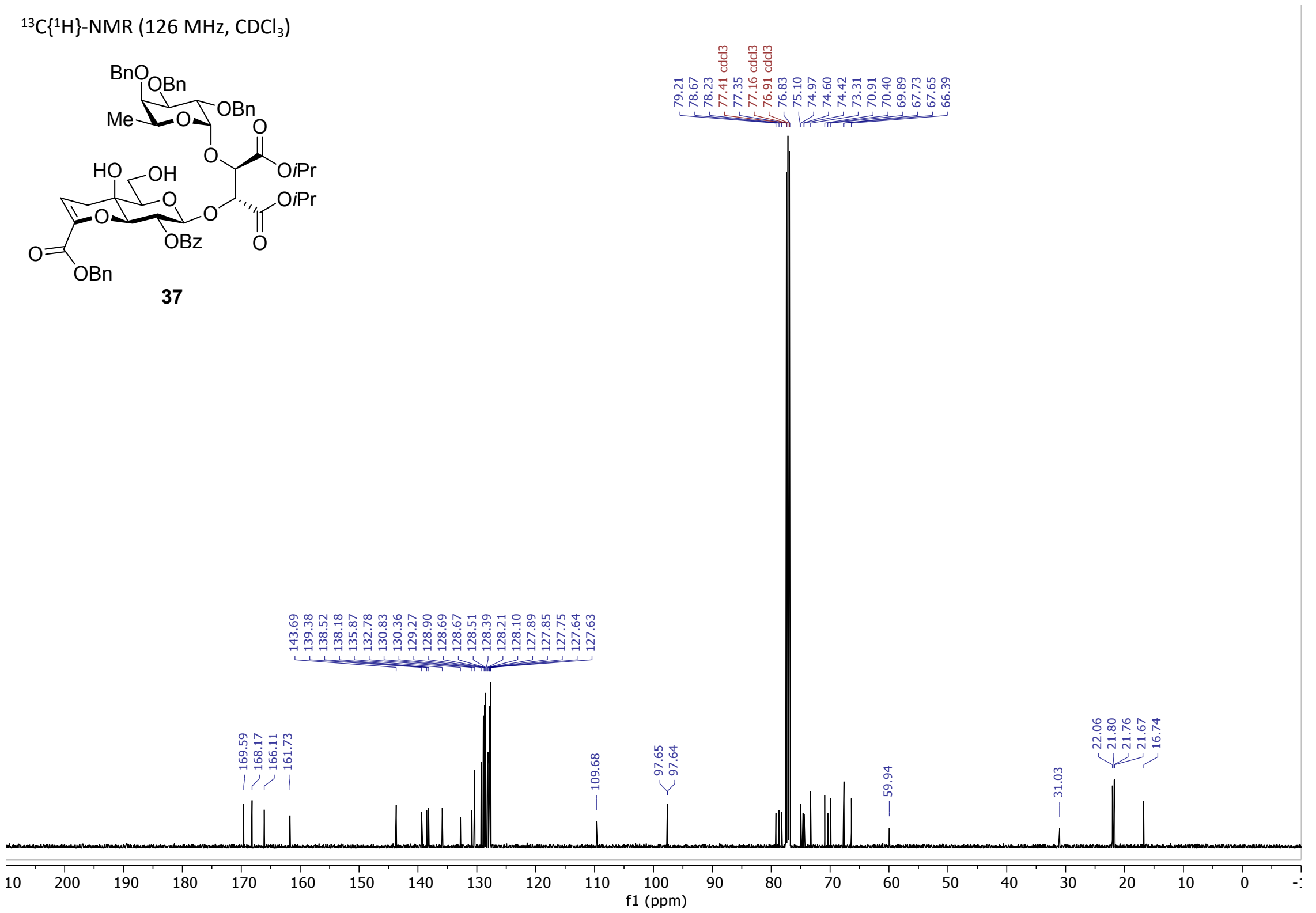


${ }^{1} \mathrm{H}-\mathrm{NMR}\left(500 \mathrm{MHz}, \mathrm{CDCl}_{3}\right.$ )

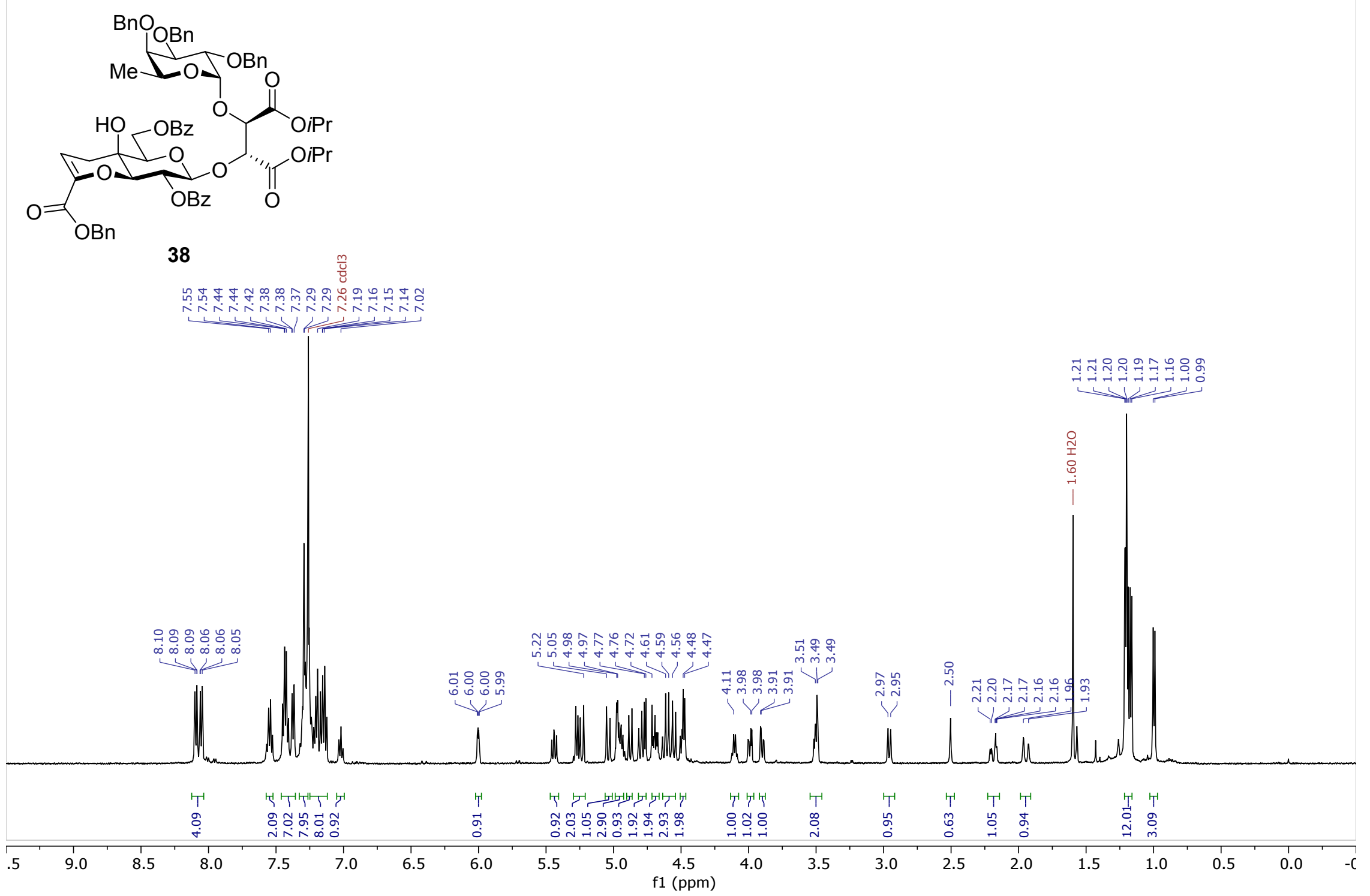




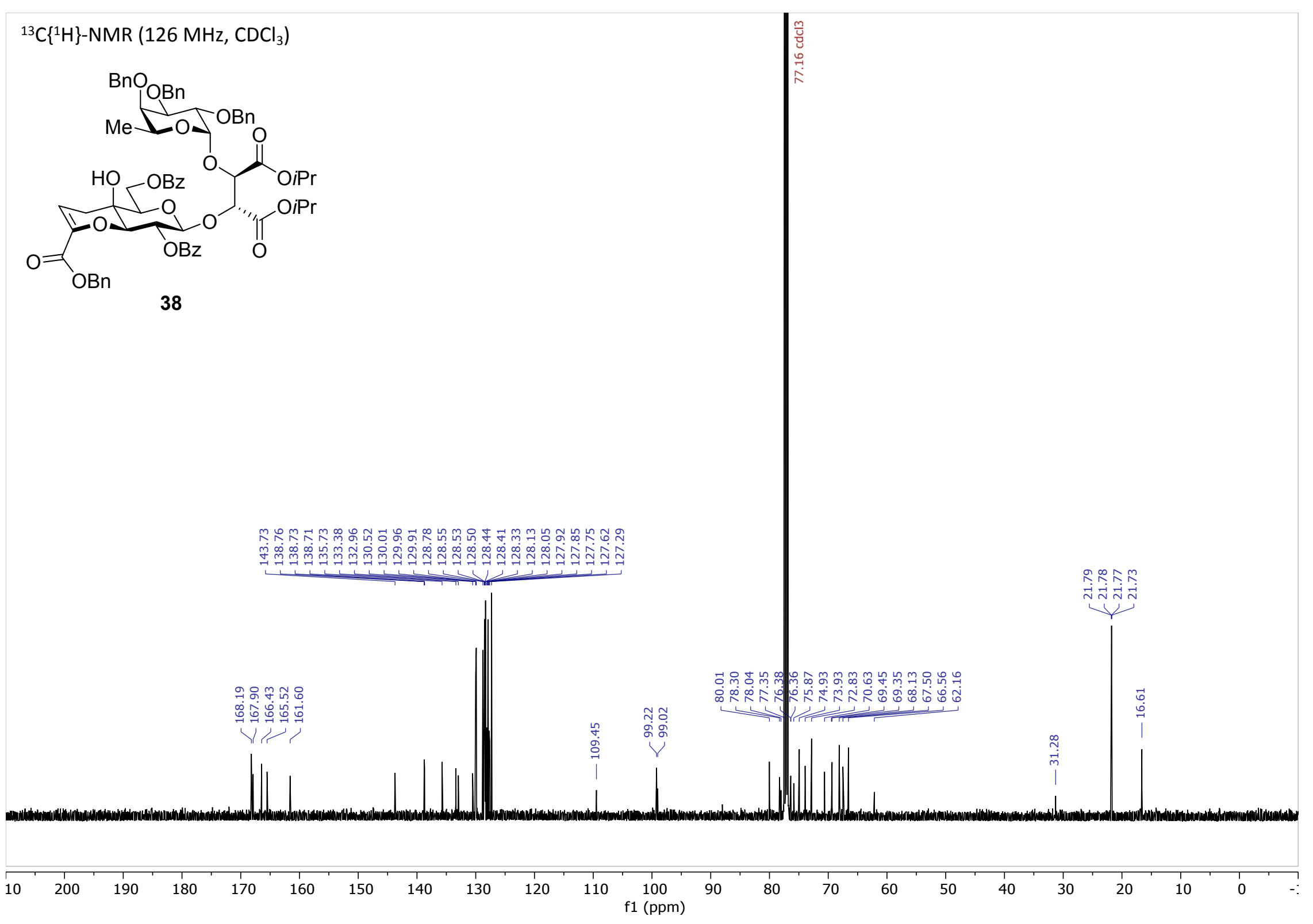




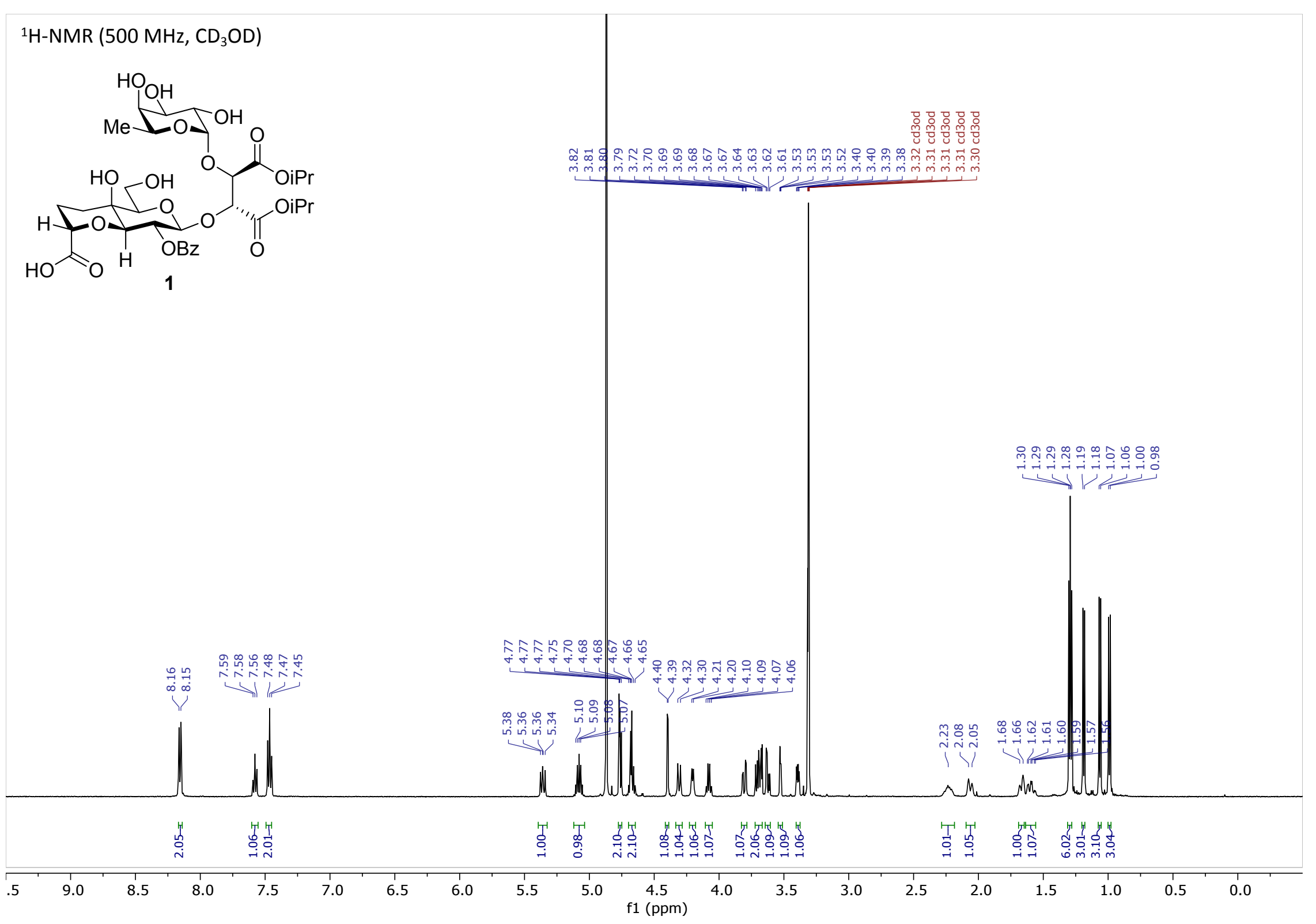




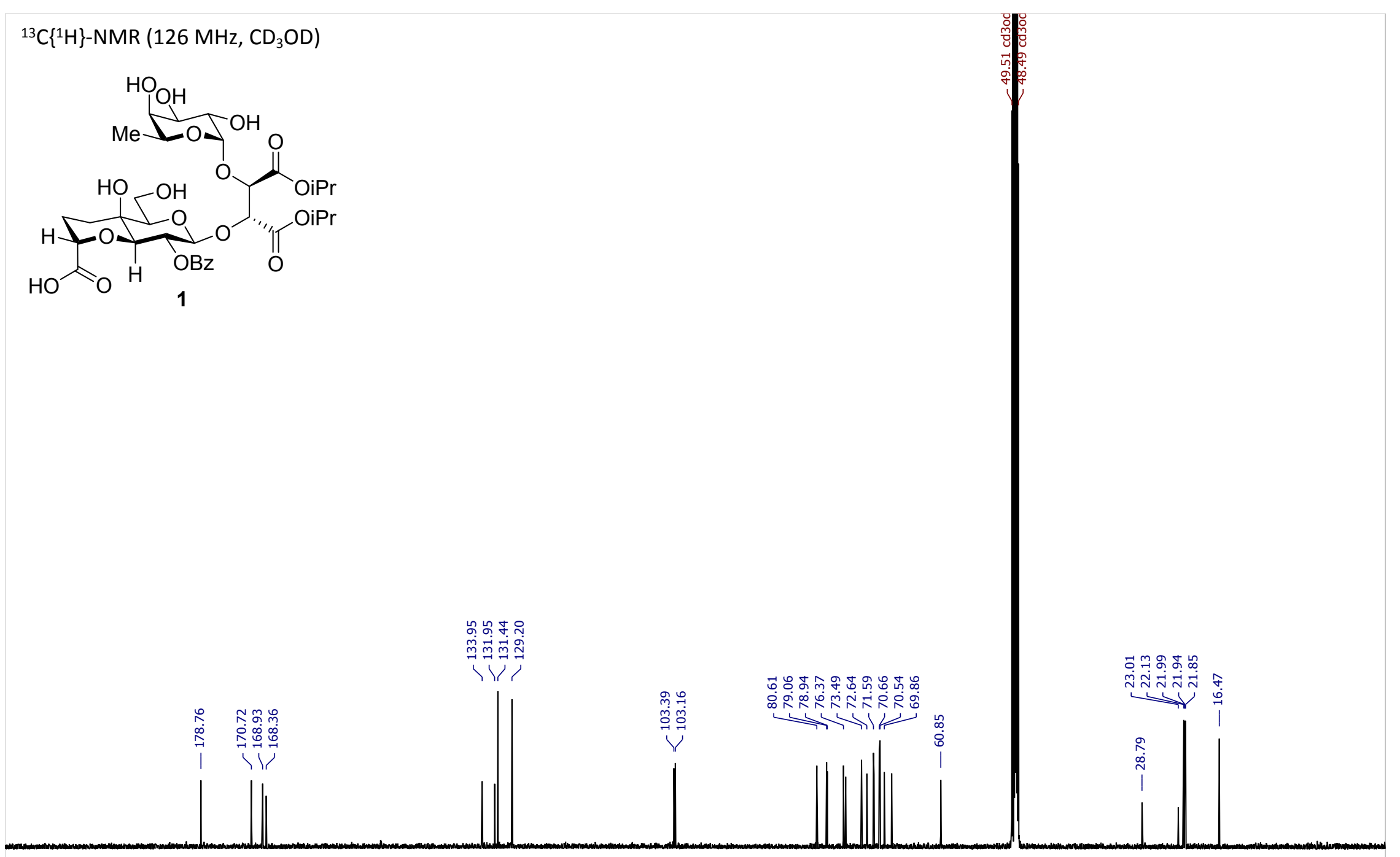$$
10
$$

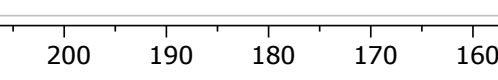

150

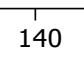


${ }^{1} \mathrm{H}-\mathrm{NMR}\left(500 \mathrm{MHz}, \mathrm{CD}_{3} \mathrm{OD}\right)$

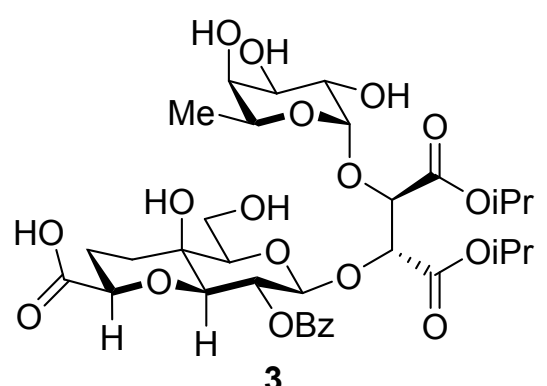

3

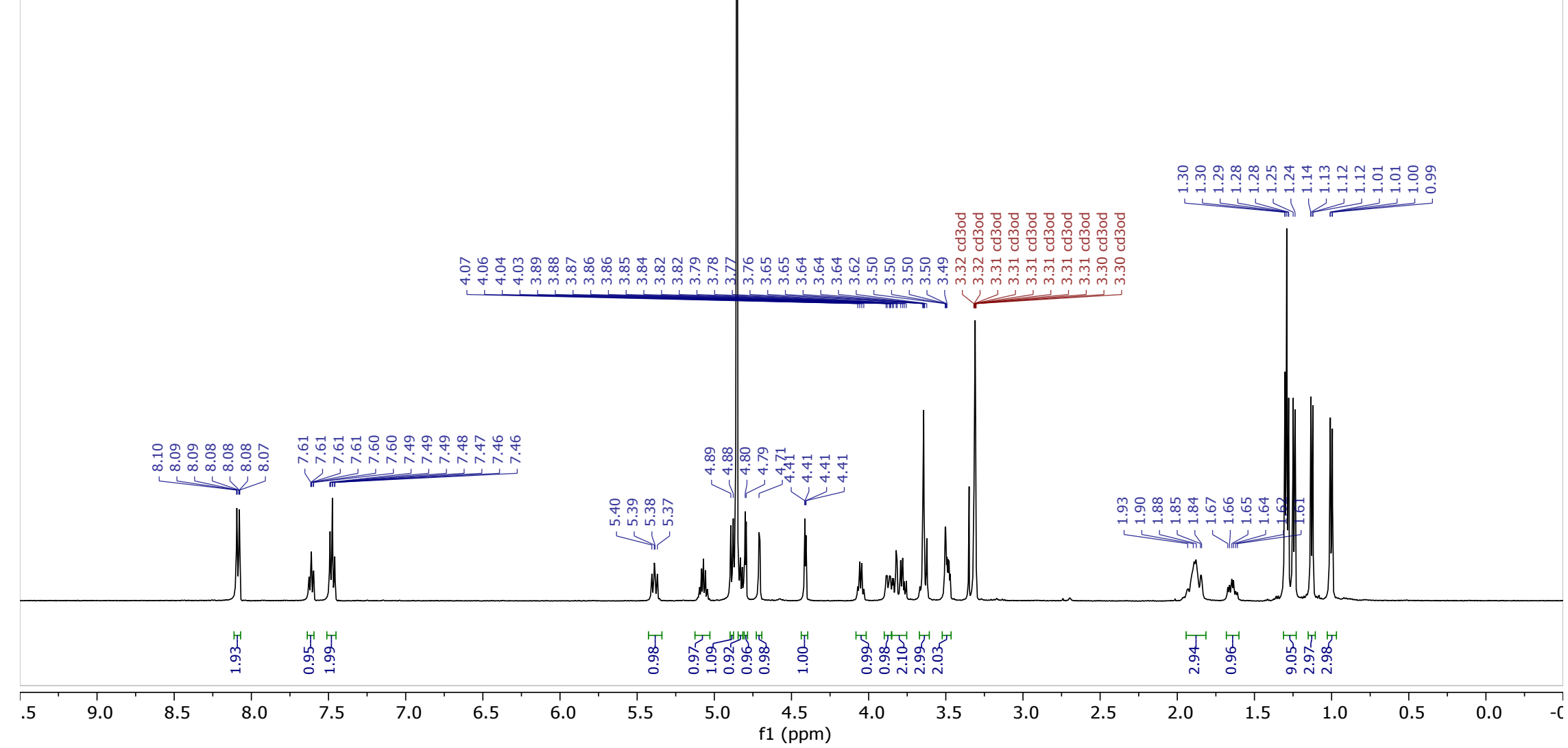



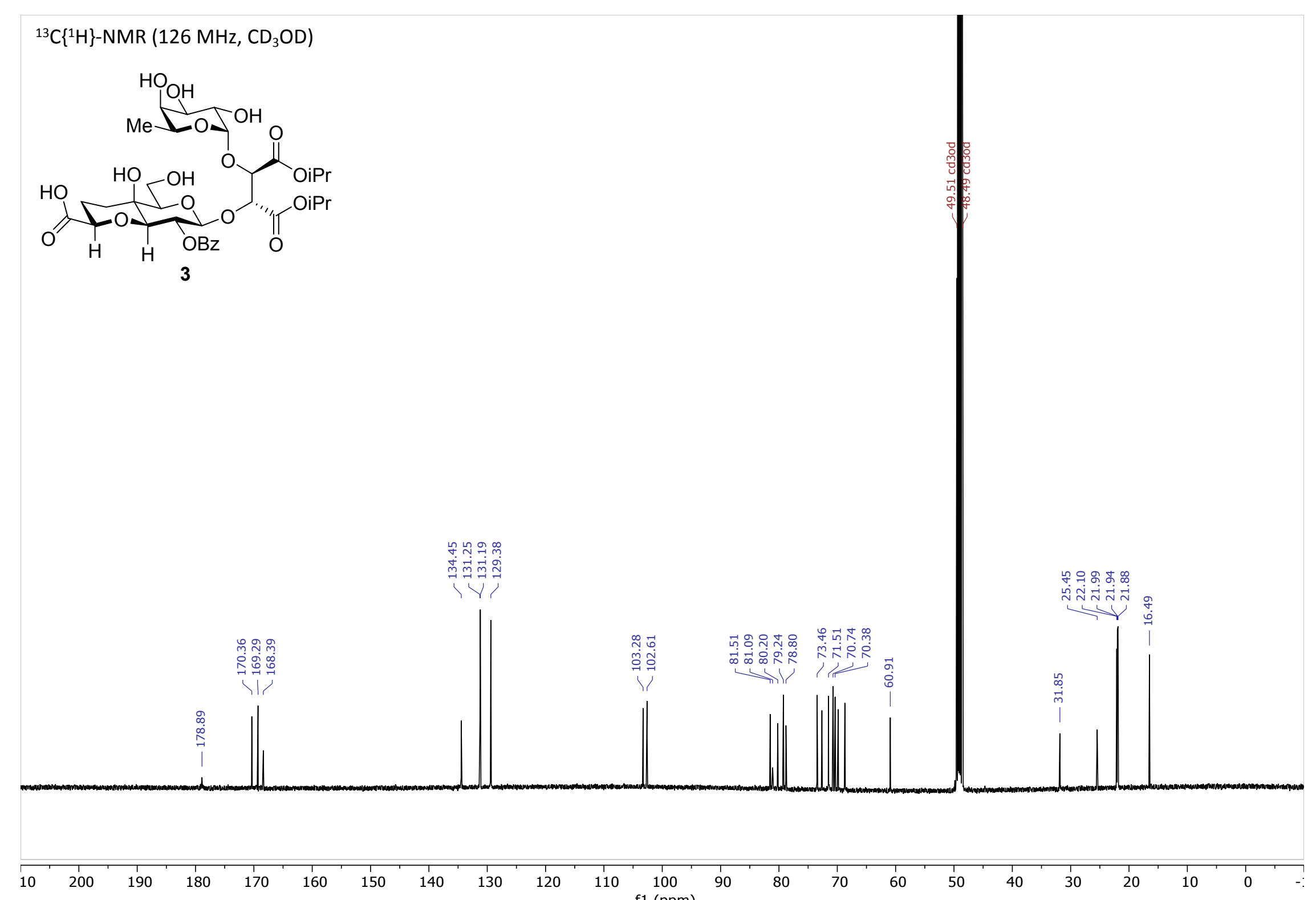
f1 (ppm)

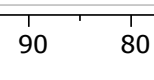
$8 0 \longdiv { 1 0 }$ $60 \quad 50$ 40 $30 \quad 20$ 
${ }^{1} \mathrm{H}-\mathrm{NMR}\left(500 \mathrm{MHz}, \mathrm{CD}_{3} \mathrm{OD}\right)$

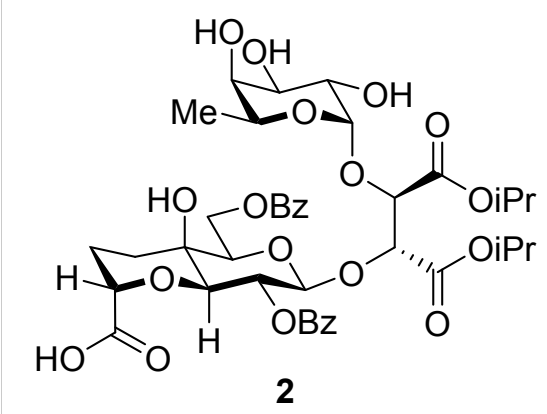

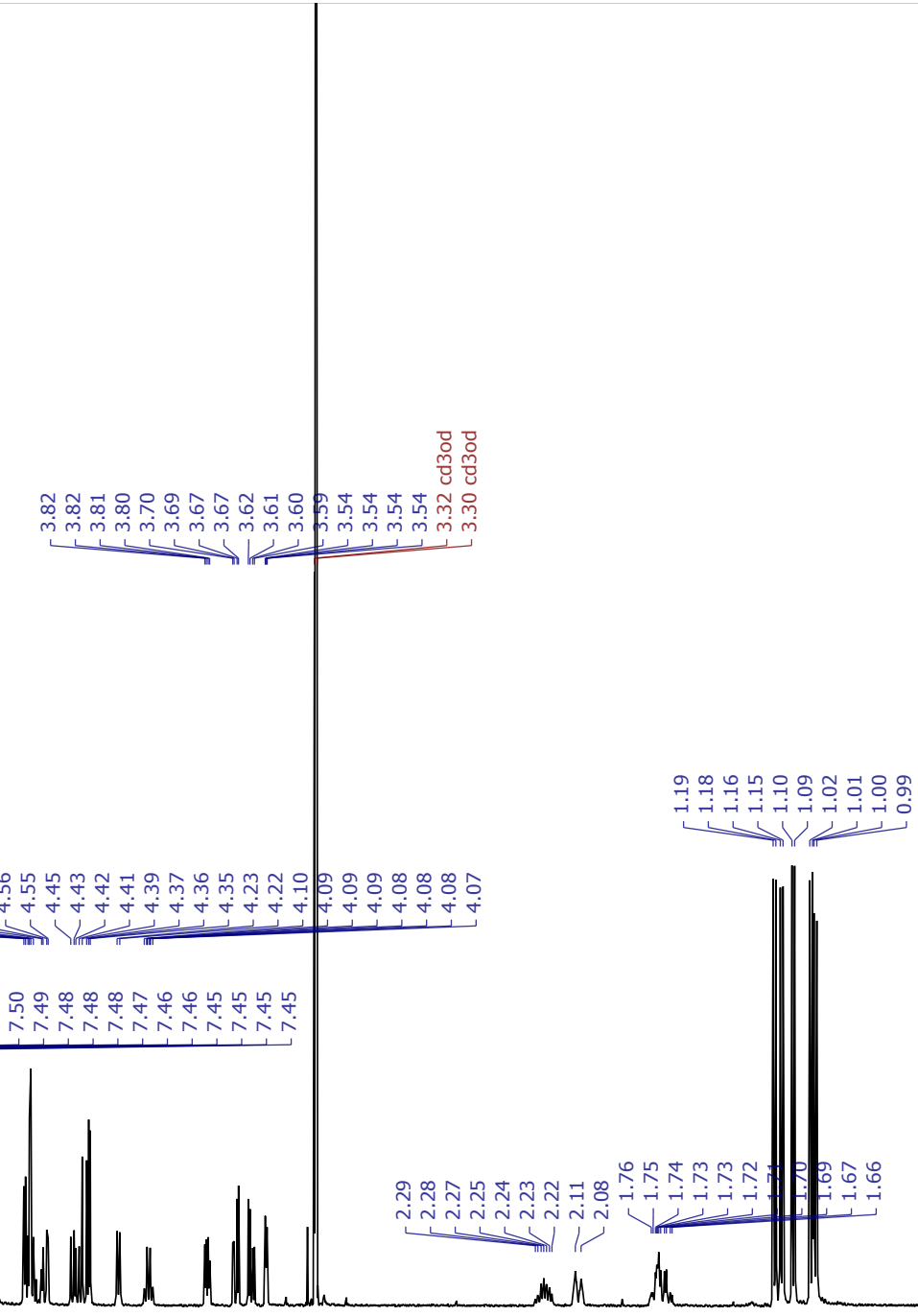

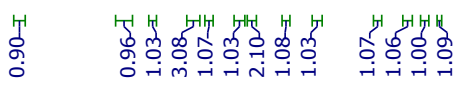

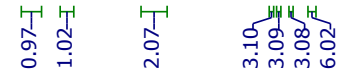

$$
.5
$$

9.0 8.5 8.0 $7.5 \quad 7.0$ 6.5 6.0

$5.5 \quad 5.0$
4.5
$\mathrm{f} 1(\mathrm{ppm})$ $4.0 \quad 3.5$ 3.0 2.5 2.0 $1.5 \quad 1.0$ $0.5 \quad 0.0 \quad-c$ 


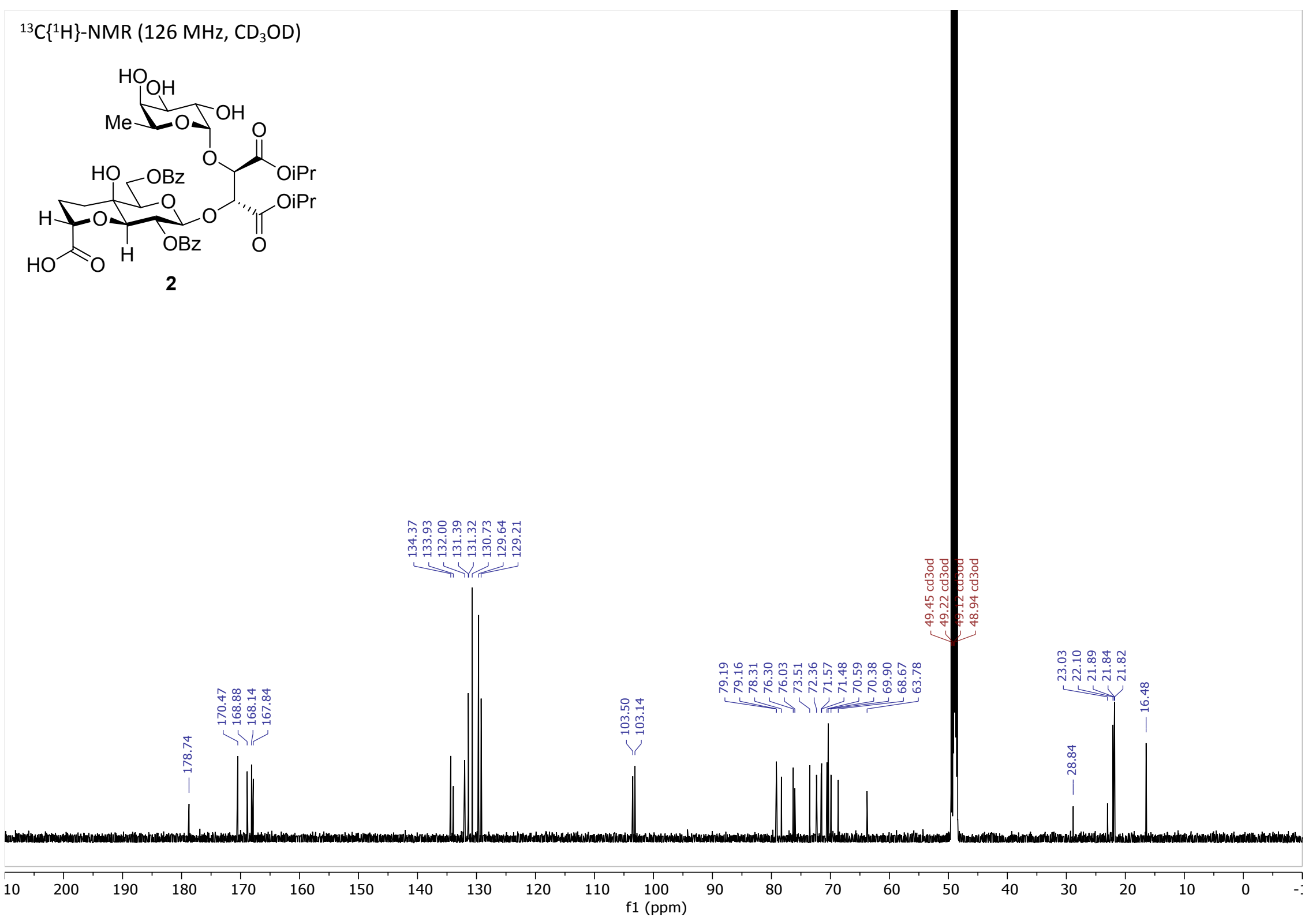


${ }^{1} \mathrm{H}-\mathrm{NMR}\left(500 \mathrm{MHz}, \mathrm{CD}_{3} \mathrm{OD}\right.$ )

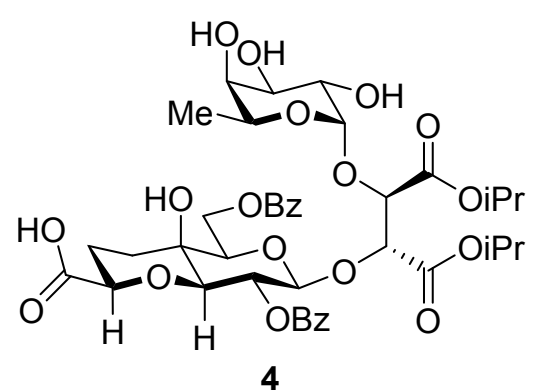

4

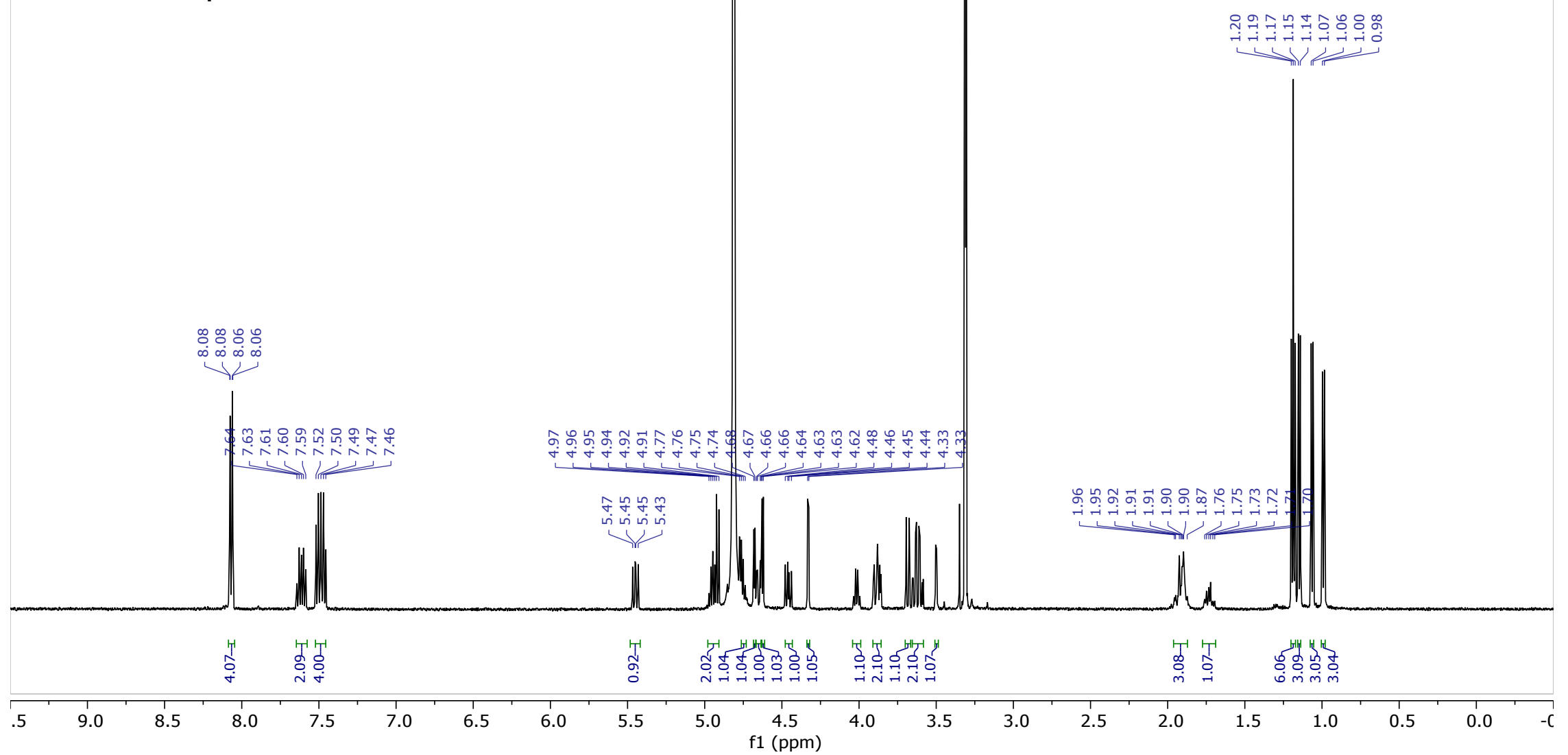



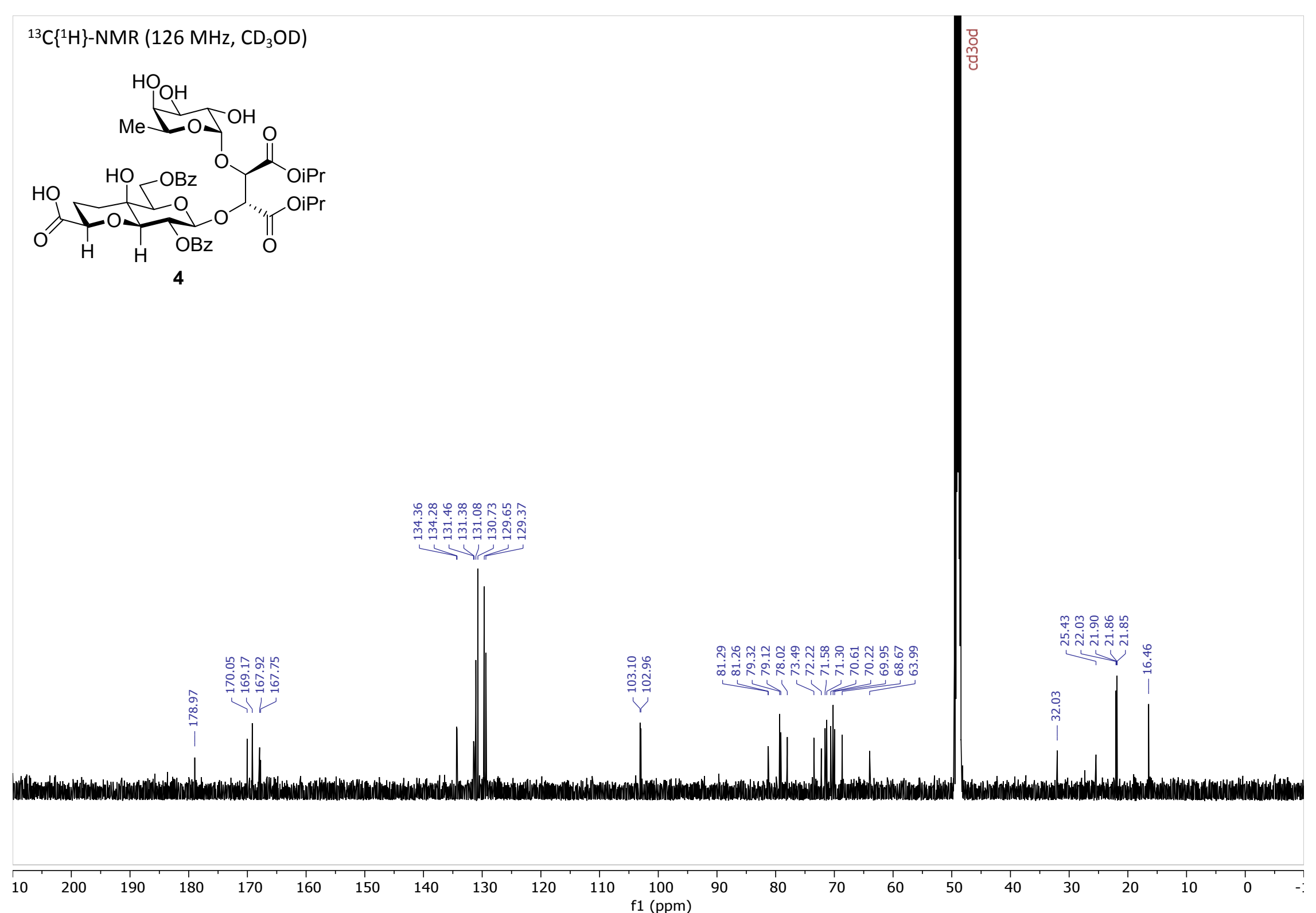


\section{Part IV: Biological Evaluations}

P-Selectin Fc HL-60 Adhesion Assay [7-8]

P-Selectin Fc $(2 \mu \mathrm{g} / \mathrm{mL})$ was incubated overnight at $4{ }^{\circ} \mathrm{C}$ in black 96 -well plate $(655090$, Greiner Bio-One). HL-60s were tagged using LeukoTracker ${ }^{\mathrm{TM}}$ (CBA-210, Cell Biolabs) for $1 \mathrm{~h}$ at $37^{\circ} \mathrm{C}$. Simultaneously the 96-well plate with P-Selectin Fc was blocked with 1:1 solution of Tris calcium buffer (pH 7.4) and StabilCoat ${ }^{\circledR}$ (S0950, Sigma-Aldrich). 10000 tagged HL-60 cells were added to each well and incubated for $1 \mathrm{~h}$ at $37^{\circ} \mathrm{C}$. Compounds $\mathbf{2 , 4}$, or sLe $\mathrm{X}^{\mathrm{X}}$ (Control) were then dissolved in Tris calcium buffer and added at a concentration range of $0.001 \mathrm{mM}$ to $5 \mathrm{mM}$ (or $10 \mathrm{mM}$ ) for 30 min at $37^{\circ} \mathrm{C}$. After carefully washing the non-adhered cells, remaining cells were photographed using Zeiss AxioObserverZ1 microscope. The number of adhered cells will decrease in a dosedependent way. Inhibition was calculated as the $100 \%$ minus binding observed in the wells.

\section{Flow Assay}

\section{Cell isolation and culture}

Primary human umbilical endothelial cells (HUVEC) were isolated as described. ${ }^{[9]}$ Briefly, the vein of an umbilical cord was cannulated, washed and endothelial cells were harvested using collagenase. Endothelial cells were seeded in T75 flasks coated with $0.2 \%$ gelatin and grown in M199 containing 16\% human serum and antibiotics. After four days, cells were split using trypsin and plated in $35 \mathrm{~mm}$ dishes coated with $0.2 \%$ gelatin. HUVEC were used in the recruitment assay once they reached confluence and exhibited a cobblestone morphology. Using this method, we have shown that HUVEC express VE-cadherin at the junctions and von Willebrand Factor (vWF) in Weibel-Palade bodies.

Human neutrophils were isolated as described. ${ }^{[10]}$ Briefly, blood was collected into heparinized syringes and red blood cells (RBC) were sedimented on Dextran 70 for 60 minutes. The leukocyte rich plasma was spun for 5 minutes at $300 \mathrm{xg}$ and the remaining RBCs were lysed by 20 second 
exposure to ice cold $0.2 \% \mathrm{NaCl}$. The cell suspension was returned to isotonicity with the addition of an equal volume of $1.6 \% \mathrm{NaCl}$ and the cell suspension was spun for 5 minutes at $300 \mathrm{xg}$. The white blood cell pellet was resuspended in $5 \mathrm{ml}$ of Hank's balanced salt solution with $0.5 \%$ human plasma albumin (HBSS/A), underlaid with $6 \mathrm{~mL}$ of Histopaque 1077 and spun for 30 minutes at $300 \mathrm{xg}$. Mononuclear cells and any remaining RBC ghosts were removed, and the granulocyte pellet was resuspended in HBSS/A and counted using a hemocytometer. Granulocytes were $>99 \%$ viable and were $>95 \%$ neutrophils with between $0-5 \%$ contaminating cells, typically eosinophils or peripheral blood mononuclear cells. Neutrophils were then diluted to 1 x $10^{6} / \mathrm{mL}$ in $\mathrm{HBSS} / \mathrm{A}$.

\section{Leukocyte rolling on histamine stimulated endothelial cells}

Endothelial cells were washed twice with HBSS and then assembled into a parallel plate flow chamber (Glycotech) to mimic the hydrodynamic conditions found in the vasculature. Neutrophils containing $10 \mu \mathrm{M}$ histamine were perfused across confluent monolayers of endothelial cells at a wall shear stress of $1 \mathrm{dyn} / \mathrm{cm}^{2}$. Interactions between neutrophils and endothelial cells were visualized using a 10x phase contrast objective and a CCD camera (Hitachi) linked to a digital recorder. Recording began as soon as neutrophils entered the chamber and continued for 10 minutes. Six fields of view were collected at 3, 5, 7 and 9 minutes

following perfusion.

The effects of sLe $\mathrm{e}^{\mathrm{x}}$ and compounds compound 2 and compound 4 were determined by adding the compounds directly to neutrophil suspension at a final concentration of $10 \mu \mathrm{M}$ prior to perfusion over the HUVEC. This ensured that the compounds were present as P-selectin was expressed on the endothelium in response to the perfused histamine.

\section{Analysis}


Data were analyzed from each of six fields of view recorded at 3, 5, 7, and 9 minutes following perfusion. Total interactions were determined by capturing two fields of view 0.33 seconds apart and subtracting the second image from the first using FIJI (ImageJ, version 2.0.0-rc-68/1.52e). ${ }^{11}$ In this way, fast moving neutrophils $(>30 \mu \mathrm{m} / \mathrm{sec})$ that were not interacting with the endothelium were eliminated. Adherent cells were determined by capturing two fields of view 5 seconds apart and subtracting the second image from the first using FIJI (ImageJ). A cell was determined to be adherent if it did not move for 5 seconds. The remaining cells were considered to be rolling. The high and low from each time point were removed and the remaining four values were averaged.
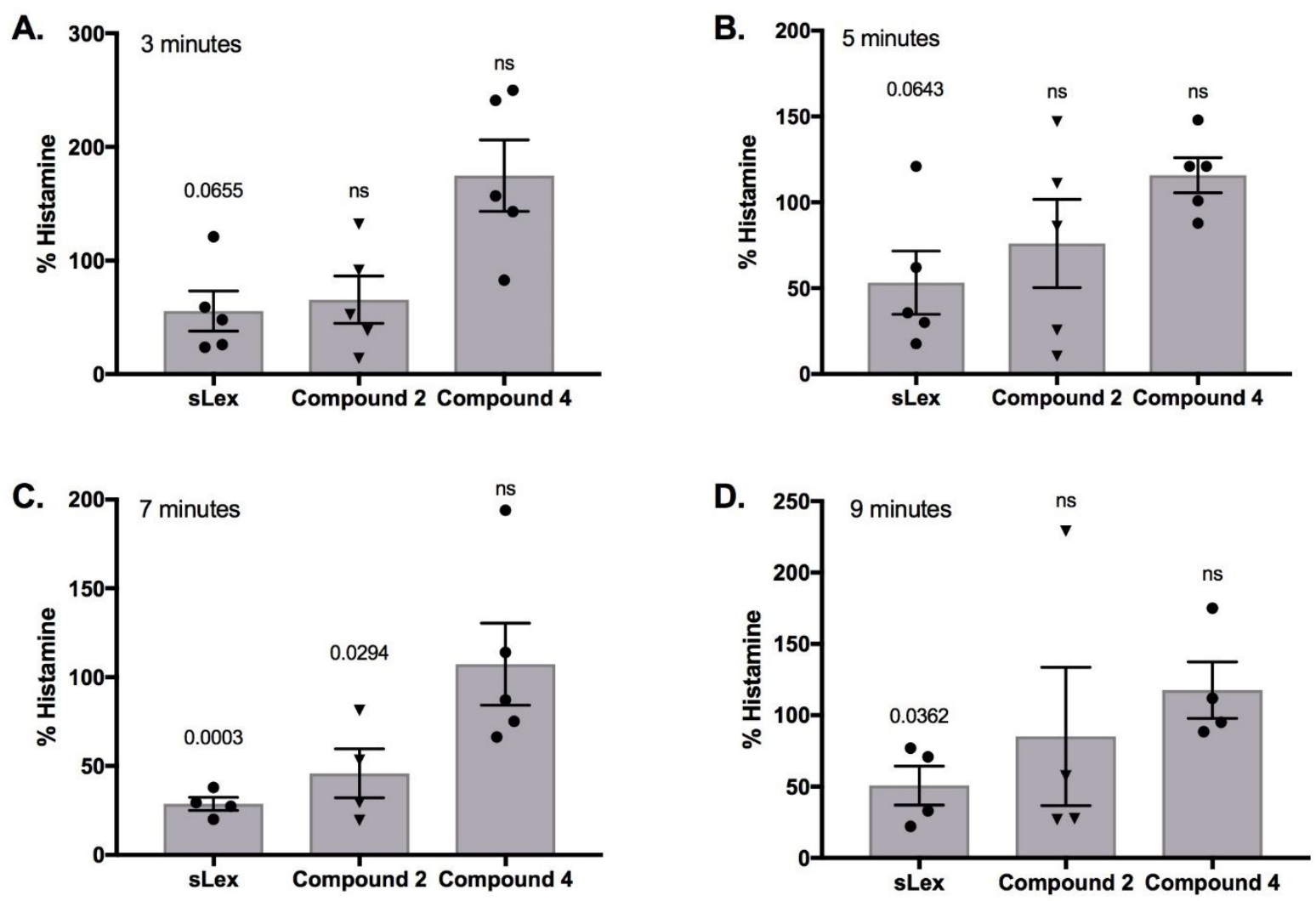

Figure S1. Effect of compounds 2 and 4, and sLe $^{\mathrm{X}}$ on rolling interactions on histaminetreated ECs. Neutrophils alone or neutrophils containing $10 \mu \mathrm{M}$ of compounds 2,4 , or sLe $\mathrm{X}^{\mathrm{X}}$ were perfused across endothelial cells and their rolling or firm adhesion behavior recorded for 10 minutes. Data were normalized for each experiment and time point and then expressed as percent histamine. Data for total interactions at 3, 5, 7 and 9 minutes (A-D) are shown as the mean $( \pm$ SEM) for 4 to 6 data points from 5 or 6 independent experiments. $p$ values represent t-test comparison to a hypothetical value of $100 \%$ and exact values are shown. 

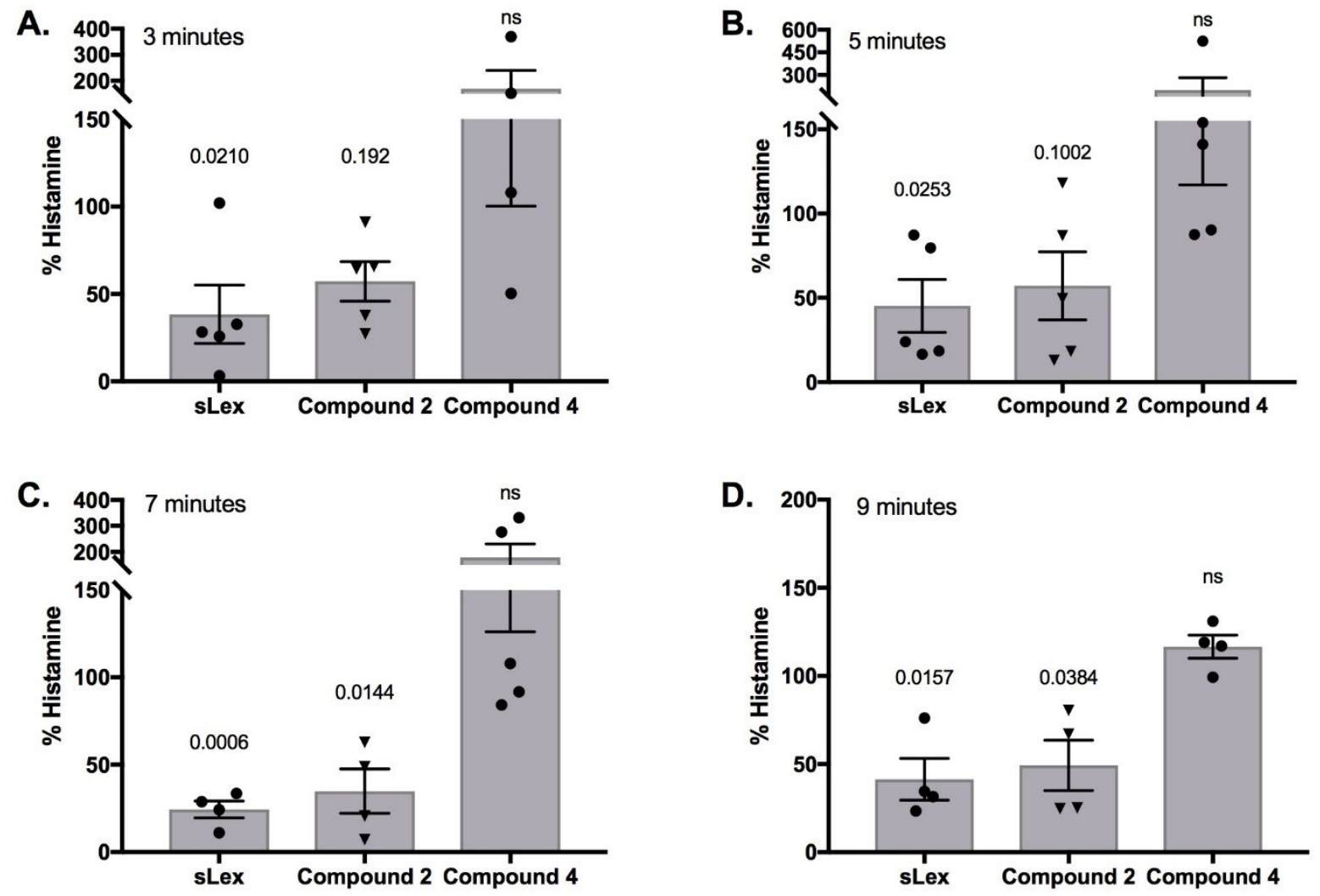

Figure S2. Effect of compounds 2 and 4, and $\operatorname{sLe}^{\mathrm{X}}$ on firm adhesion on histamine-treated ECs. Neutrophils alone or neutrophils containing $10 \mu \mathrm{M}$ of compounds 2,4 , or $\mathrm{sLe}^{\mathrm{X}}$ were perfused across endothelial cells and their rolling or firm adhesion behavior recorded for 10 minutes. Data were normalized for each experiment and time point and then expressed as percent histamine. Data for total interactions at 3, 5, 7 and 9 minutes (A-D) are shown as the mean ( \pm SEM) for 4 to 6 data points from 5 or 6 independent experiments. $p$ values represent $t$-test comparison to a hypothetical value of $100 \%$ and exact values are shown. 


\section{References:}

1) Gaussian 09, Revision A.02, M. J. Frisch, G. W. Trucks, H. B. Schlegel, G. E. Scuseria, M. A. Robb, J. R. Cheeseman, G. Scalmani, V. Barone, G. A. Petersson, H. Nakatsuji, X. Li, M. Caricato, A. Marenich, J. Bloino, B. G. Janesko, R. Gomperts, B. Mennucci, H. P. Hratchian, J. V. Ortiz, A. F. Izmaylov, J. L. Sonnenberg, D. Williams-Young, F. Ding, F. Lipparini, F. Egidi, J. Goings, B. Peng, A. Petrone, T. Henderson, D. Ranasinghe, V. G. Zakrzewski, J. Gao, N. Rega, G. Zheng, W. Liang, M. Hada, M. Ehara, K. Toyota, R. Fukuda, J. Hasegawa, M. Ishida, T. Nakajima, Y. Honda, O. Kitao, H. Nakai, T. Vreven, K. Throssell, J. A. Montgomery, Jr., J. E. Peralta, F. Ogliaro, M. Bearpark, J. J. Heyd, E. Brothers, K. N. Kudin, V. N. Staroverov, T. Keith, R. Kobayashi, J. Normand, K. Raghavachari, A. Rendell, J. C. Burant, S. S. Iyengar, J. Tomasi, M. Cossi, J. M. Millam, M. Klene, C. Adamo, R. Cammi, J. W. Ochterski, R. L. Martin, K. Morokuma, O. Farkas, J. B. Foresman, and D. J. Fox, Gaussian, Inc., Wallingford CT, 2016.

2) Zhao, Y.; Truhlar, D. G., The M06 suite of density functionals for main group thermochemistry, thermochemical kinetics, noncovalent interactions, excited states, and transition elements: two new functionals and systematic testing of four M06-class functionals and 12 other functionals. Theor. Chem. Acc. 2007, 120, 215.

3) Zhao, Y.; Truhlar, D. G., Density functionals with broad applicability in chemistry. Acc. Chem. Res. 2008, 41, 157.

4) Cossi, M.; Scalmani, G.; Rega, N.; Barone, V., New developments in the polarizable continuum model for quantum mechanical and classical calculations on molecules in solution. J. Chem. Phys. 2002, 117, 43.

5) Zhao, Y.; Truhlar, D. G., Computational characterization and modeling of buckyball tweezers: density functional study of concave-convex pi...pi interactions. Phys. Chem. Chem. Phys. 2008, 10, 2813.

6) Ribeiro, R. F.; Marenich, A. V.; Cramer, C. J.; Truhlar, D. G., Use of solution-phase vibrational frequencies in continuum models for the free energy of solvation. J. Phys. Chem. B. 2011, $115,14556$.

7) Ghoshal, P.; Rajendran, M.; Odo, N.; Ikuta, T., Glycosylation inhibitors efficiently inhibit Pselectin-mediated cell adhesion to endothelial cells. PLoS One 2014, 9, e99363.

8) Calosso, M.; Tambutet, G.; Charpentier, D.; St-Pierre, G.; Vaillancourt, M.; Bencheqroun, M.; Gratton, J. P.; Prevost, M.; Guindon, Y., Acyclic tethers mimicking subunits of polysaccharide ligands: selectin antagonists. ACS Med. Chem. Lett. 2014, 5, 1054.

9) Ganguly, A.; Zhang, H.; Sharma, R.; Parsons, S.; Patel, K. D., Isolation of human umbilical vein endothelial cells and their use in the study of neutrophil transmigration under flow conditions. J. Vis. Exp. 2012, 66, e4032.

10) Chakrabarti, S.; Patel, K. D., Regulation of matrix metalloproteinase-9 release from IL-8stimulated human neutrophils. J. Leukoc. Biol. 2005, 78, 279. 
11) Schindelin, J.; Arganda-Carreras, I.; Frise, E.; Kaynig, V.; Longair, M.; Pietzsch, T.; Preibisch, S.; Rueden, C.; Saalfeld, S.; Schmid, B.; Tinevez, J. Y.; White, D. J.; Hartenstein, V.; Eliceiri, K.; Tomancak, P.; Cardona, A., Fiji: an open-source platform for biological-image analysis. Nat. Methods 2012, 9, 676. 